\title{
AN ASSET MANAGEMENT ASSESSMENT MODEL FOR STATE DEPARTMENTS OF TRANSPORTATION
}

\author{
By \\ STEVEN ROBERT COOKSEY \\ Bachelor of Science in Civil Engineering \\ Oklahoma State University \\ Stillwater, OK \\ 2007
}
Submitted to the Faculty of the
Graduate College of the Oklahoma State University in partial fulfillment of the requirements for the Degree of MASTER OF SCIENCE

May, 2009 


\section{AN ASSET MANAGEMENT ASSESSMENT MODEL FOR STATE DEPARTMENTS OF TRANSPORTATION}

Thesis Approved:

Dr. Hyunseok (David) Jeong

\begin{tabular}{c} 
Thesis Adviser \\
Dr. Garold Oberlender \\
Dr. Tyler Ley \\
Dr. A. Gordon Emslie \\
\hline Dean of the Graduate College
\end{tabular}




\section{ACKNOWLEDGEMENTS}

I would like to thank my advisor, Dr. Hyunseok (David) Jeong for all of his patience and direction throughout this entire study. His guidance cannot be overlooked.

I would also like to thank my committee members, Dr. Garold Oberlender and Dr. Tyler Ley for all of their assistance in this research process.

My deepest gratitude goes to my parents, Bob and Peggy Cooksey, not only for encouraging me during my research, but for all the support they have freely given me both as an undergraduate and graduate student at Oklahoma State University. I could not have done it without them. 


\section{TABLE OF CONTENTS}

Chapter

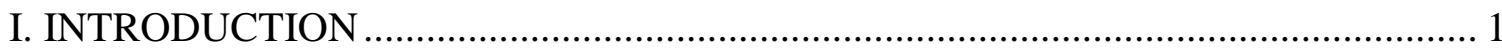

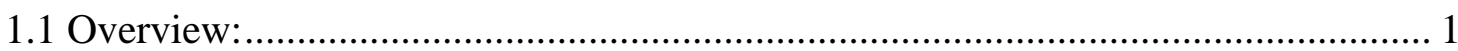

1.2 Problem Statement:

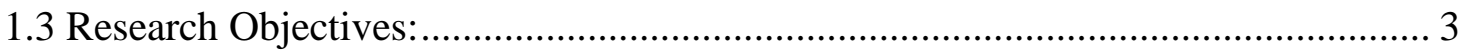

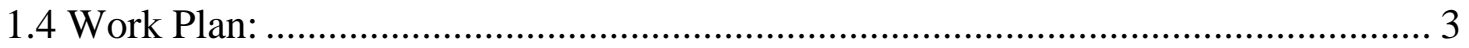

1.4.1 Literature Review: ............................................................................... 4

1.4.2 Analysis of Practices and Policies: ............................................................ 5

1.4.3 Interview leading DOTs:.......................................................................... 5

1.4.4 Select Assessment Methodologies:............................................................... 5

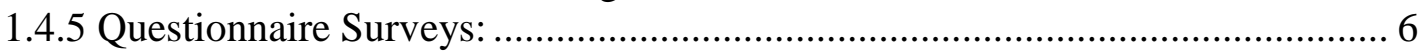

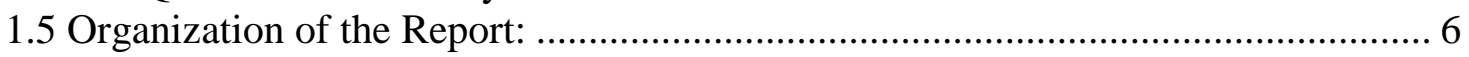

II. REVIEW OF LITERATURE......................................................................... 7

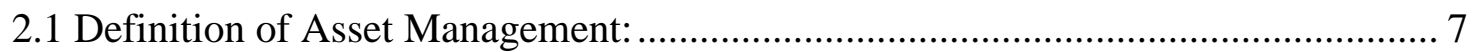

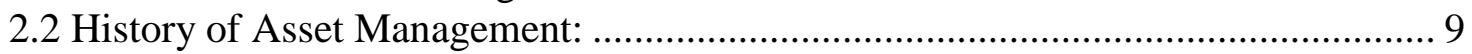

2.3 Current Status of Asset Management: ............................................................. 10

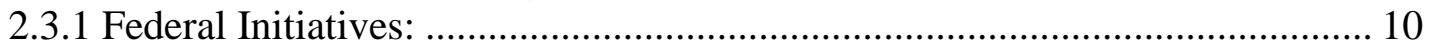

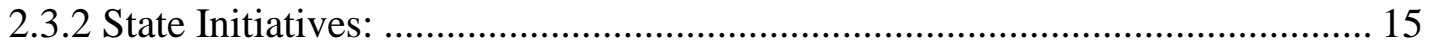

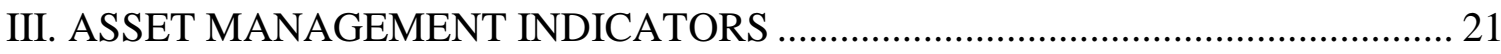

3.1 Introduction:

3.2 Asset Management Framework: ....................................................................... 21

3.3 Asset Management Culture:.............................................................................. 22

3.3.1 Asset Management Champions: ................................................................. 23

3.3.2 Perception of Asset Management: ............................................................... 24

3.3.3 Effective Communication: .................................................................... 25

3.3.4 State Legislative Mandate:...................................................................... 26

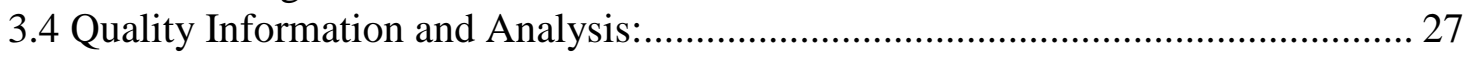

3.4.1 Asset Inventory: .................................................................................... 27

3.4.2 Common Referencing System: ............................................................ 28

3.4.3 Up-to-Date Condition Data:....................................................................... 29

3.4.4 Data Management and Analysis: ................................................................. 31

3.5 Policy Goals and Objectives (Major Indicator): .................................................. 32

3.5.1 Policy Goals and Objectives (Sub-Indicator): ................................................... 33 
3.5.2 Performance Measures \& Targets:........................................................... 35

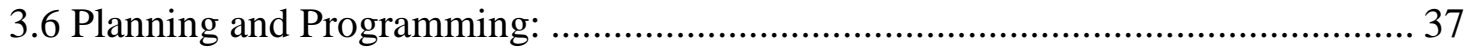

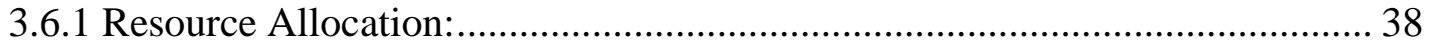

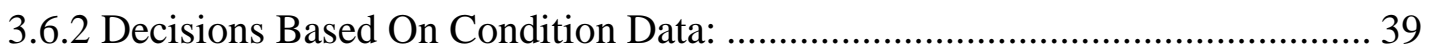

3.7 Program Delivery (Major Indicator): .............................................................. 40

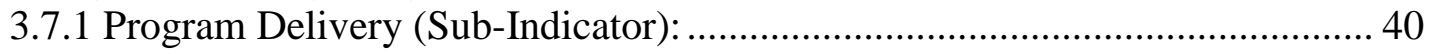

IV. ASSESSMENT OF ASSET MANAGEMENT INDICATORS ............................ 43

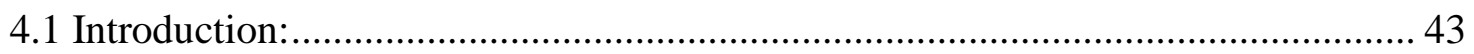

4.2 The Analytic Hierarchy Process: ..................................................................... 44

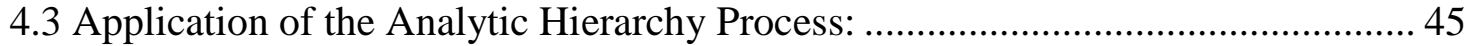

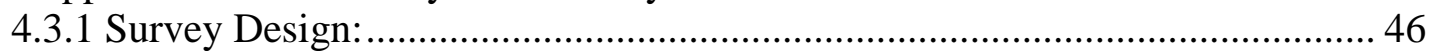

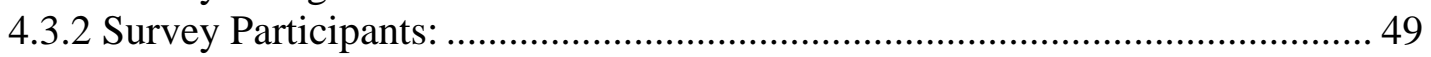

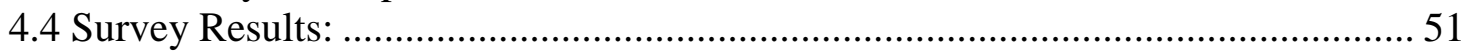

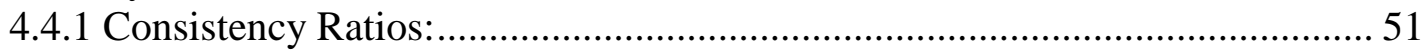

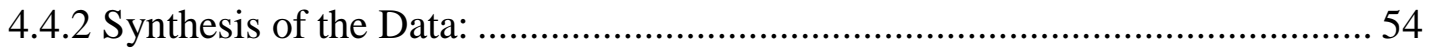

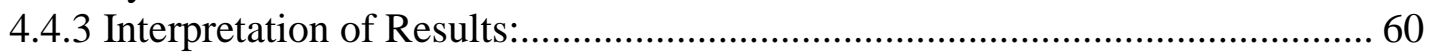

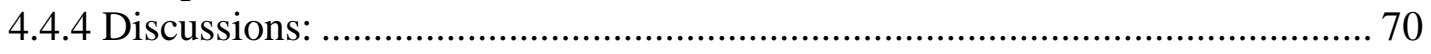

V. ASSET MANAGEMENT ASSESSMENT MODEL ............................................... 72

5.1 Introduction:

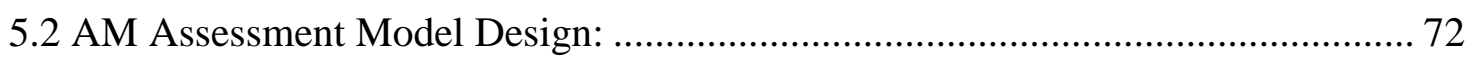

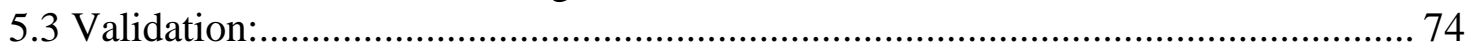

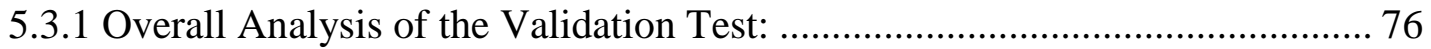

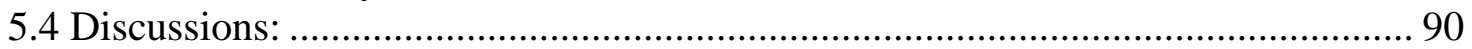

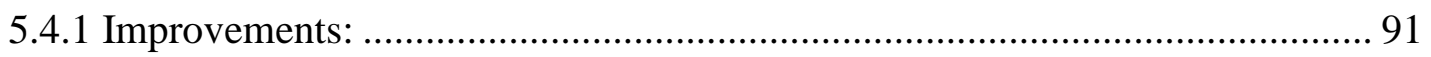

5.4.2 Ideal Application of the AM Assessment Model:........................................ 92

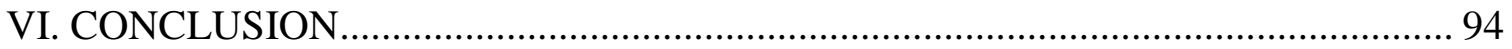

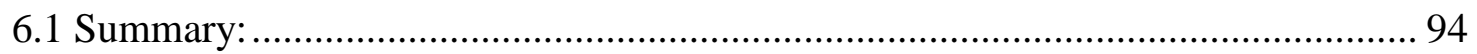

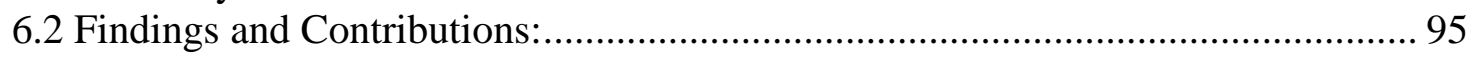

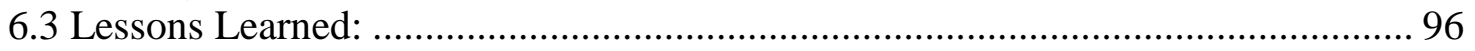

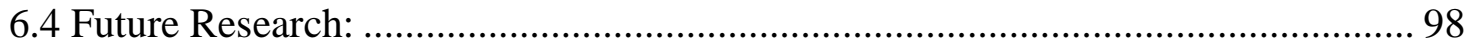

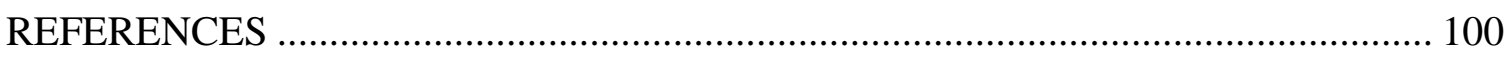

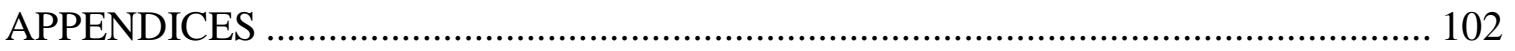

APPENDIX A: INDICATOR RANKING SURVEY ............................................ 103

APPENDIX B: ASSET MANAGEMENT ASSESSMENT MODEL, VALIDATION 111

APPENDIX C: ASSET MANAGEMENT ASSESSMENT MODEL .......................... 114 


\section{LIST OF TABLES}

Table

Page

\section{CHAPTER IV}

Table 4.1: The Fundamental Scale (Saaty and Vargas 2001) ....................................... 47

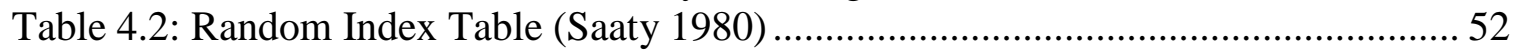

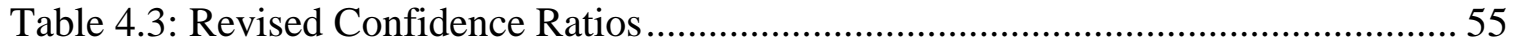

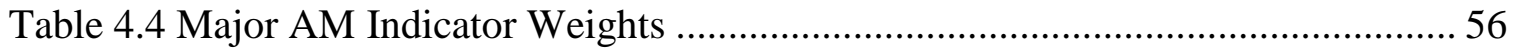

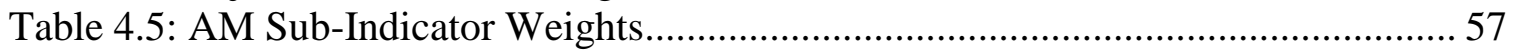

Table 4.6: Total Weights for AM Sub-Indicators ....................................................... 58

\section{CHAPTER V}

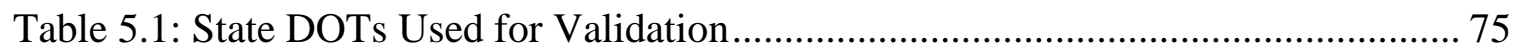

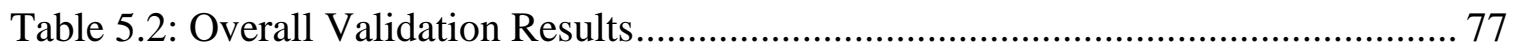

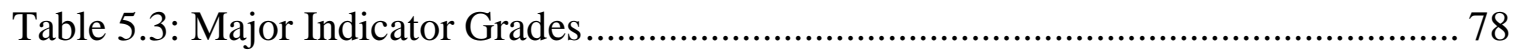

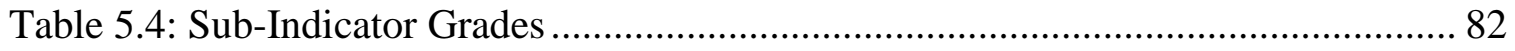

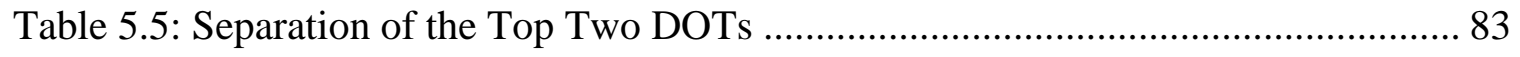

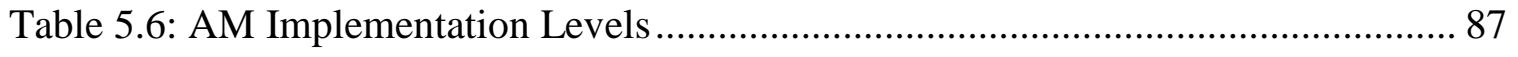




\section{LIST OF FIGURES}

Figure $\quad$ Page

\section{CHAPTER I}

Figure 1.1: Research Objectives and Work Tasks ................................................... 4

\section{CHAPTER II}

Figure 2.1: Asset Management Framework (Cambridge Systematics 2002) .................. 12

\section{CHAPTER III}

Figure 3.1: Asset Management Framework/Indicators.

\section{CHAPTER IV}

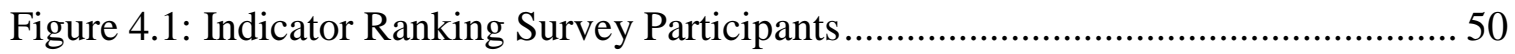

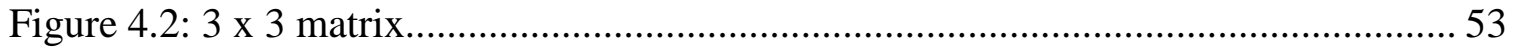

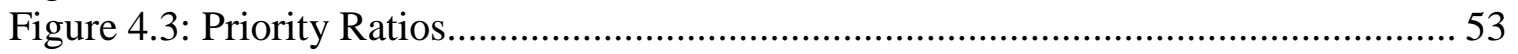

Figure 4.4: Matrix of Absolute Differences................................................................ 54

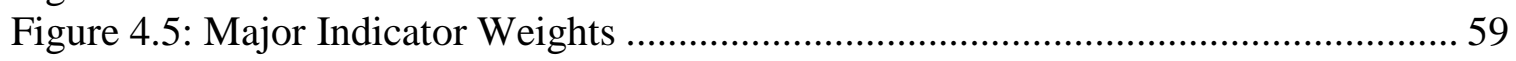

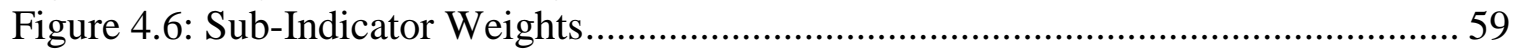

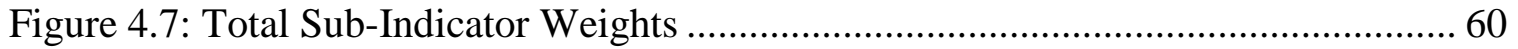

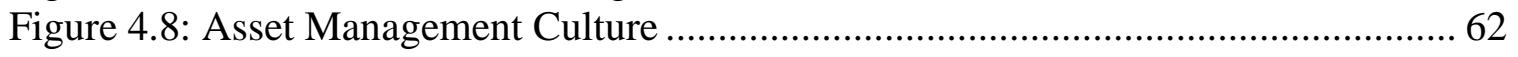

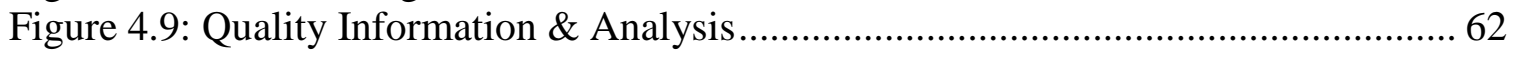

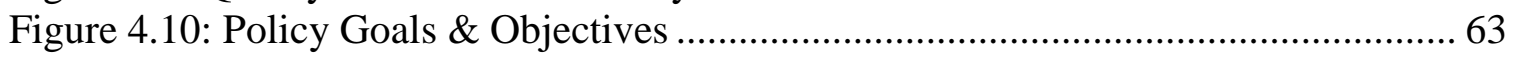

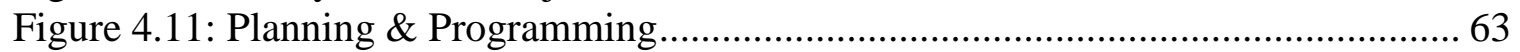

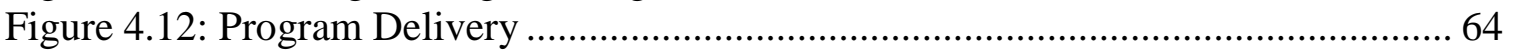

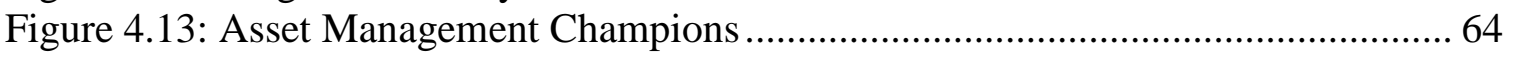

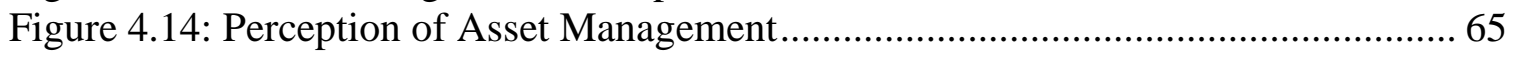

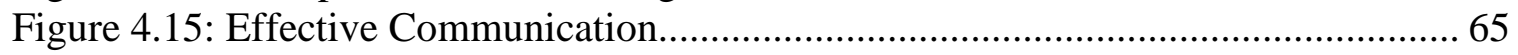

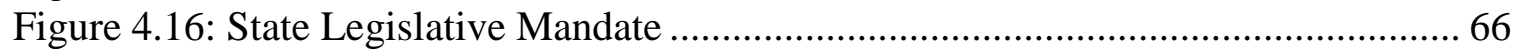

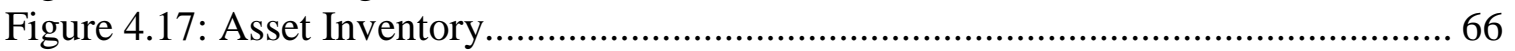

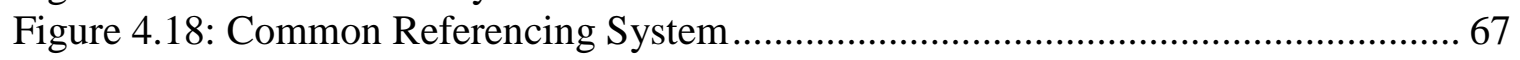

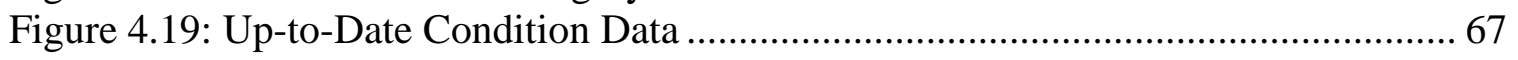

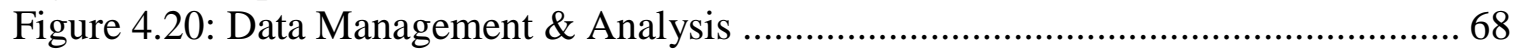

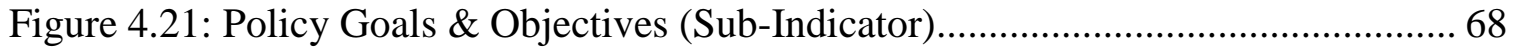

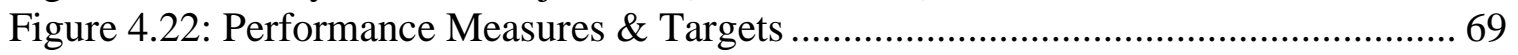

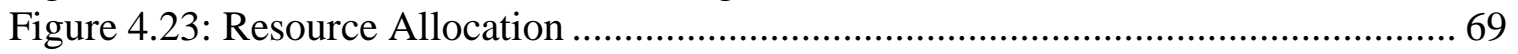

Figure 4.24: Decisions Based on Condition Data .................................................. 70 


\section{CHAPTER V}

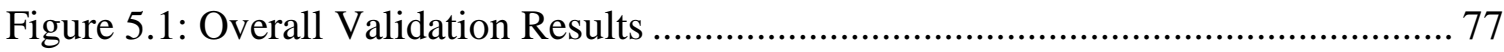

Figure 5.2: Asset Management Culture Grades ........................................................... 79

Figure 5.3: Quality Information \& Analysis Grades ................................................. 79

Figure 5.4: Policy Goals \& Objectives Grades ..................................................... 80

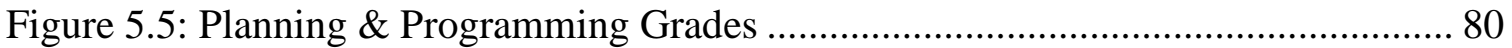

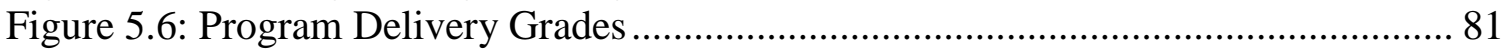

Figure 5.7: Separation of the Top Two DOTs ......................................................... 83

Figure 5.8: Policy Goals \& Objectives Grades (Sub-Indicator) .................................... 84

Figure 5.9: Performance Measures \& Targets Grades (Sub-Indicator) ............................ 84

Figure 5.10: Program Delivery Grades (Sub-Indicator) ............................................ 85

Figure 5.11: Asset Inventory Grades (Sub-Indicator) ................................................. 85

Figure 5.12: Decisions Based on Condition Data (Sub-Indicator) ................................ 86

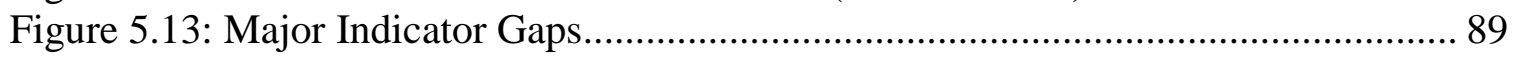

Figure 5.14: Weighted Gaps for the Major Indicators .............................................. 89

Figure 5.15: Weighted Gaps for Quality Information \& Analysis Sub-Indicators.......... 90 


\section{CHAPTER I}

\section{INTRODUCTION}

\subsection{Overview:}

Transportation Asset Management (AM) is often described simply as a decisionmaking framework. It is an all-encompassing strategy that examines all of the transportation assets and manages them as one unit. For many years, however, the only form of AM at State Departments of Transportation (DOTs) consisted of two separate systems: bridge and pavement management. Historically, vertical and horizontal communication within many State DOTs has been limited, and little, if any data exchange has taken place between the two management systems. This resulted in not being able to evaluate trade-offs between different types of assets (FHWA 1999). Traditionally, many states planned their projects on a "worst first" basis and managed their assets in a tactical rather than strategic fashion. As a result, the Federal Highway Administration (FHWA) created the Office of Asset Management in 1998. This, along with numerous other research initiatives sought to create the framework for AM that could be applied to State DOTs.

The National Cooperative Highway Research Program (NCHRP) (A program administered by the Transportation Research Board (TRB) and sponsored by the American Association of State Highway and Transportation Officials (AASHTO) and FHWA) describes the principles of AM as a policy driven, performance-based system 
that includes analysis of options and tradeoffs whose decisions are based on quality information, all of which require monitoring to provide clear accountability and feedback (Cambridge Systematics 2006). FHWA (1999) adds: “An Asset Management decisionmaking framework is guided by performance goals, covers an extended time horizon, draws from economics as well as engineering, and considers a broad range of assets that include physical as well as human resources." Based on these statements, it is obvious that transportation AM goes beyond just looking at the individual parts of infrastructure, but rather examines the system as a whole. As a result of the research performed by NCHRP, a framework for transportation AM has been made available for State DOTs to model.

\subsection{Problem Statement:}

While there is a well defined and accepted AM framework for State DOTs, there have not been any studies that have developed a model that measures the level of AM implementation within a DOT for a benchmarking purpose. Is it possible to measure this for every DOT based on a common benchmark? The difficulty in developing a set of common AM best practices is that every DOT is different. Each may have a different organizational structure as well as different set of priorities that guide their decisionmaking. However, one assumption that this study relies on is that there are a common set of factors critical to the success of an ideal AM system. These factors or "indicators" must be identified through the careful evaluation of successful AM practices among DOTs. If this is possible, a better picture of AM may be developed. 


\subsection{Research Objectives:}

The objective of this study is to develop an Asset Management Assessment Model for State DOTs in the United States. The specific objectives include:

A) Identify and document current practices and policies used by different DOTs to implement AM.

B) Identify AM indicators that represent the most critical components of an ideal AM system within a State DOT.

C) Weigh the AM indicators based on their level of importance in implementing an ideal AM system within a State DOT.

D) Develop and apply the AM Assessment Model to State DOTs in order to evaluate the model's effectiveness.

E) Recommend the most appropriate AM Assessment Model.

\subsection{Work Plan:}

There are five major work tasks that are required to complete this research. They include:

1) Literature review

2) Analysis of practices and policies

3) Interview leading DOTs in AM

4) Select assessment methodologies

5) Questionnaire Surveys 
These work tasks are used as a step-by-step process to achieve the research objectives. They are the support tools necessary to complete the project. Their relationships with the research objectives are illustrated in Figure 1.1 and described below.

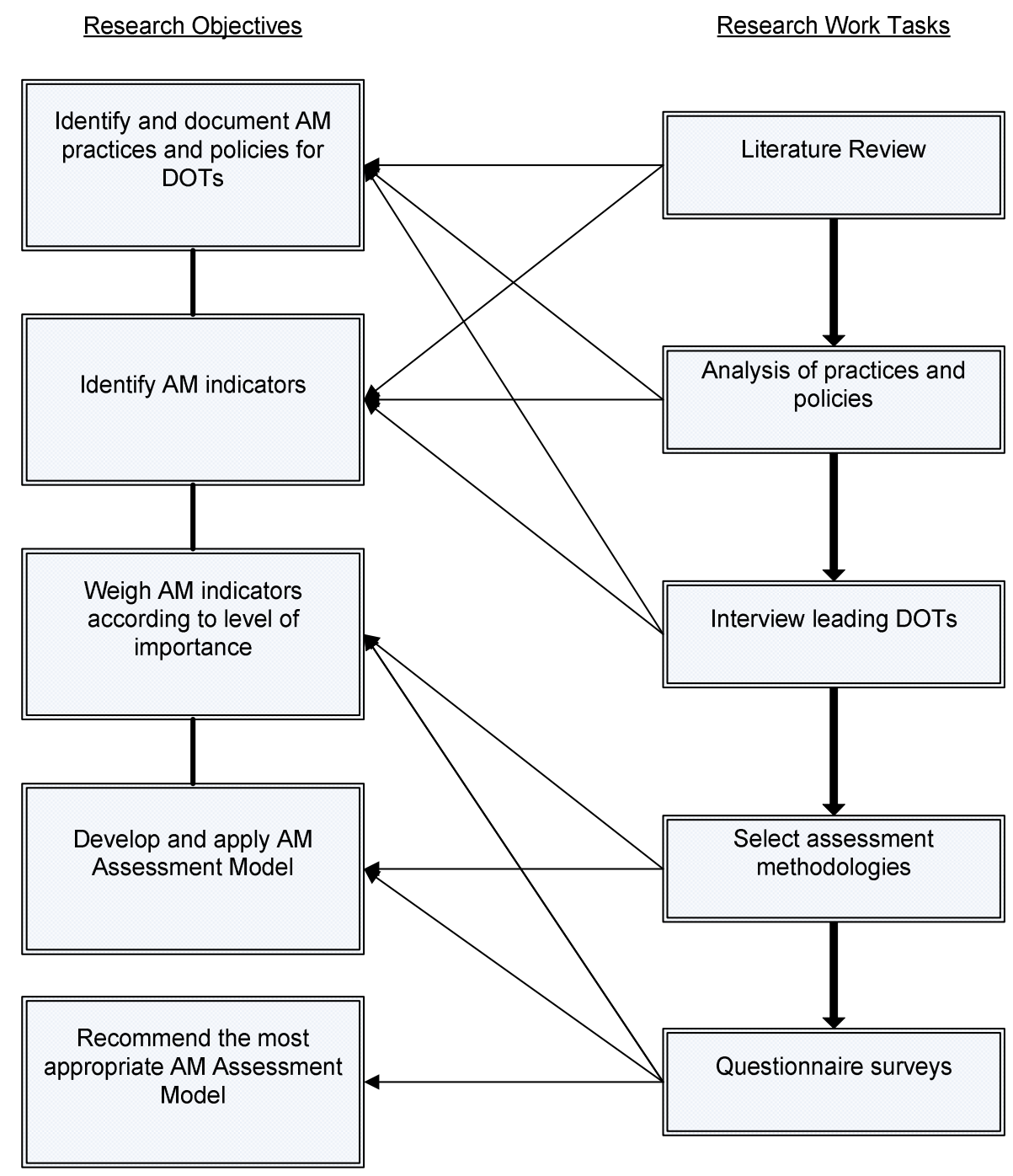

Figure 1.1: Research Objectives and Work Tasks

\subsubsection{Literature Review:}

The literature review allows the research team to gain knowledge in the area of transportation AM by reviewing current journals and research publications related to the 
subject matter. The literature review will be used primarily to support the first two objectives, but is useful throughout the entire study.

\subsubsection{Analysis of Practices and Policies:}

Once the literature review is completed, careful examination of current AM practices will help identify which of these are best suited for an ideal AM system. This work task also supports the first two objectives; especially in identifying critical AM indicators.

\subsubsection{Interview leading DOTs:}

As a result of the literature review, the leading DOTs in AM will be determined. Visits to five of the top DOTs in AM will be made in order to collect detailed information about what their current AM practices are as well as help identify which indicators are necessary for an ideal AM system.

\subsubsection{Select Assessment Methodologies:}

Following the identification of the AM indicators, a methodology will be needed to accurately quantify the relative importance of each indicator. Because the indicators are subjective, a methodology that can convert subjective values into quantifiable weights is desired. Once this is completed, a method to collect information about the weights as well as administer the AM Assessment Model will need to be determined. 


\subsubsection{Questionnaire Surveys:}

The first questionnaire will be used to collect data in order to determine the weights for each of the AM indicators. This survey will list each of the AM indicators and require the participant to use the selected methodology to evaluate each indicator. Based on this information, weights for each indicator will be developed.

The second survey will be used to administer the AM Assessment Model. Each participant will respond to a set of statements related directly to the AM indicators. Based on their responses, an assessment of their agency's AM implementation level will be determined.

\subsection{Organization of the Report:}

Chapter II discusses previous research done in fields directly related to this topic. It provides a definition of $\mathrm{AM}$, and the current status of AM activity both at the federal and state levels. Chapter III identifies the major AM indicators and sub-indicators. Each of these is described in detail as to why they are critical components of AM. Chapter IV discusses the methodology used to quantify the major indicators and sub-indicators. The results of these weights are expressed and analyzed. Chapter V explains how the AM Assessment Model was designed, administered, and validated. Chapter VI summarizes the research, explains the contributions, and discusses future research topics. 


\section{CHAPTER II}

\section{REVIEW OF LITERATURE}

\subsection{Definition of Asset Management:}

Transportation AM for State DOTs has several definitions. The "Asset Management Primer" by the FHWA defines AM as a "decision-making framework that is guided by performance goals" (FHWA 1999). It should provide an economic assessment of tradeoffs between alternative improvements and investment strategies. It also states that an AM system should be customer focused, mission driven, system oriented, longterm in outlook, accessible, user friendly, and flexible (FHWA 1999). The objective of AM has been described as helping an agency "make the right investments at the right time" (Guerre et al. 2005). AM should be comprehensive, "focus on customer and community needs, provide quality services and a commitment to excellence to ensure that assets remain productive" (AASHTO 1997).

Perhaps the most complete definition of $\mathrm{AM}$ is contained in the research performed by NCHRP. NCHRP Report 551 defines AM as a "strategic approach to managing transportation infrastructure" (Cambridge Systematics 2006) that is based on the principles described in the "Transportation Asset Management Guide" (Cambridge Systematics 2002): 
Policy-Driven - Decisions on infrastructure management reflect policy goals and objectives that define asset condition, levels of performance, and quality of services to meet customer needs and broader economic, community, and environmental goals.

Performance-Based - Goals and objectives must be tied to clear measures of performance. Targets established for these performance measures will guide decisions through the analysis of options, setting of priorities, and program budgeting and implementation.

Analysis of Options and Tradeoffs - Competition for scarce resources and interrelationships among decisions in different investment areas and affecting different assets all argue for considering options and evaluating the tradeoffs among alternatives.

Decisions Based on Quality Information - Choices among options during program development, project selection, and program and service delivery are based on their relative costs and consequences in meeting performance targets. Objective, high-quality information is applied at each step, using analytic methods and decision criteria that are consistent with policy goals and objectives and an agency's business process.

Monitoring to Provide Clear Accountability and Feedback - Performance measures are monitored and reported, providing feedback on the effectiveness of transportation investments and services, work accomplished, and program and service delivery. 


\subsection{History of Asset Management:}

During the 1990s, the only form of AM consisted of pavement and bridge management systems. Under this system, investment decisions were project driven, and asset preservation was frequently the by-product of facility expansion and new construction. These management systems also planned and programmed their projects on a "worst first" basis. This approach to asset management was tactical rather than strategic. Also, there was little, if any, data exchange between the management systems and as a result they were not able to evaluate trade-offs between various classes of assets such as highways versus bridges (FHWA 1999).

As a result, FHWA created the "Asset Management Primer" (FHWA 1999) in 1999 after the 1998 reorganization effort which created the Office of Asset Management. The purpose of the primer was to educate transportation officials as to why AM was necessary. The primer states that "Asset Management has come of age because of (1) changes in the transportation environment, (2) changes in public expectations, and (3) extraordinary advances in technology" (FHWA 1999).

The primer describes that AM is necessary because of system demands, personnel constraints, increased budget demands, and accountability to the public (FHWA 1999):

- Because the Interstate Highway System is completed and aging, the system demands are directed towards maintenance and reconstruction as well as system performance and reliability.

- Personnel constraints refer to downsizing that many State DOTs are facing. Because of the loss of professional staff, DOTs are forced to prioritize their work 
and are more likely to focus on management functions rather than the day-to-day technical functions which are increasingly being outsourced.

- Budget demands arise from increased usage, costs, and needed upgrades. These factors are constraints on the transportation budget which is already competing for funding with other publicly supported programs.

- Increased accountability to the public is necessary because of public skepticism of government along with an increasing preference for using private-sector management approaches in the public sector which has led to demands that the government should be more accountable and operate more like a private business.

\subsection{Current Status of Asset Management:}

In recent years $\mathrm{AM}$ has become a popular topic among transportation officials. It is an area that most have heard of, but fewer are successfully implementing. There are numerous workshops held around the country each year to educate officials about the best practices in AM. Much research has been done to help outline the principles of AM so that state agencies can adopt these principles into their practice. The next two sections will outline what AM initiatives have been developed at the federal level and the state level.

\subsubsection{Federal Initiatives:}

As previously mentioned, FHWA initiated one of the first steps towards AM at the federal level when the Office of Asset Management was established in 1998. Shortly thereafter, in 1999, FHWA produced the "Asset Management Primer." According to the 
primer, AM is a philosophy that "focuses on the benefits of investment, as well as its costs, and takes a comprehensive view of the entire portfolio of transportation resources. Asset Management is an improved way of doing business that responds to an environment of increasing system demands, aging infrastructure, and limited resources" (FHWA 1999). This is an important point for transportation officials to realize because once they buy into this philosophy, they can begin to take steps to implement this system within their agency. In order to implement this new AM system, a framework was developed at the federal level for State DOTs to model.

Research sponsored by AASHTO and FHWA with the TRB produced NCHRP Project 20-24(11). Two tasks of this project were to: propose a generic framework for transportation AM that can be adopted by member states, and develop an AASHTO "Guide for Transportation Asset Management" (The "Guide"). The "Guide," developed in 2002, provides agencies with guidance for implementing AM concepts and principles within their business processes (Cambridge Systematics 2002). It was completed so that State DOTs could follow the correct steps to begin AM implementation.

The "Guide" first reviews basic AM principles and the necessary framework. It also defines AM as a process of resource allocation and utilization. This process consists of policy goals, objectives, and performance measures; planning and programming; program delivery; and systems monitoring and performance results, all of which is supported by quality information and analysis. These principles are represented in a framework shown in Figure 2.1. The "Guide" stresses that this framework was intentionally created to be generic so that any agencies can adopt the basic principles while being able to adjust them to their individual needs. The "Guide" also allows 
agencies to identify strengths, weaknesses, and areas in need of improvement through the application of a self-assessment test. Next, it defines the scope of AM and establishes roles and responsibilities for an agency. In the following chapters, the "Guide" covers each of the four areas of transportation AM (policy goals, planning and programming, program delivery, and systems monitoring) by reviewing key topics, best practices, and practical implementation steps. Finally, it covers implementation, which is how to perform tasks identified in the AM action plan, track progress, and update the plan as fundamental changes occur.

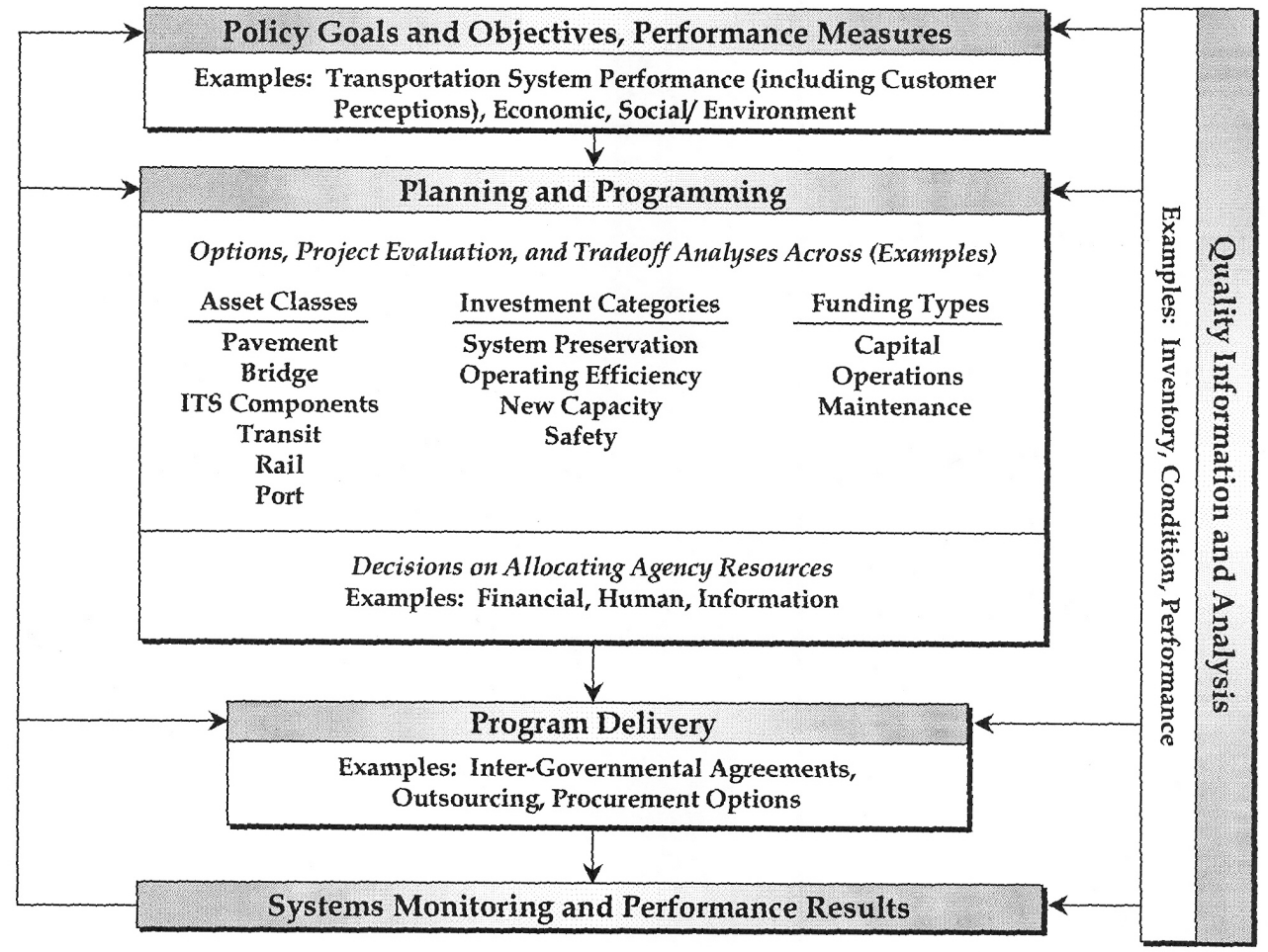

Figure 2.1: Asset Management Framework (Cambridge Systematics 2002) 
There have been numerous studies performed at the federal level to stimulate the understanding and use of AM for State DOTs. These studies include analyzing AM as a system as well as examining its components. In 2001, FHWA produced the "Data Integration Primer" to promote the use of data integration as a part of AM. One of the goals of this report was to help agencies understand the importance of reliable data in AM. "Useful and reliable data are central to a fully functioning Asset Management process" (FHWA 2001). This primer also discusses the benefits of data integration, how to integrate data, and challenges to data integration; all within a total AM perspective.

The "Life-Cycle Cost Analysis Primer" was produced in 2002 by FHWA to address the use life-cycle cost analysis (LCCA) as a part of AM. This primer examines why LCCA should be used, how it should be applied, as well as issues that LCCA faces as a tool for transportation agencies. LCCA is tool that gives decision-makers the ability to determine the most cost-effective solution "for a transportation investment requirement and is therefore a natural fit within the Asset Management framework" (FHWA 2002).

"Analytical Tools for Asset Management" was published in 2005 as a result of NCHRP Project 20-57. The purpose of this study was to develop a set of analytical tools that could assist State DOTs in making tradeoff decisions while managing their agency's assets. The first phase of this study consisted of a needs assessment that reviewed existing analytical tools and documented their capabilities and limitations. One of the primary conclusions from the first phase found that "many existing analytical tools are not being used to their full potential to influence investment decision-making" (Cambridge Systematics 2005). The second phase of the study focused on developing two tools (AssetManager NT and AssetManager PT) to fill in the gaps found as a result 
of the first phase. Even though these tools were developed to assist transportation agencies in decision-making, the study also pointed out needed improvements for each tool as well as some of the remaining gaps in analytical tools for AM.

One of the most important factors of $\mathrm{AM}$ is the use performance measures to guide decision-making on how to invest limited resources. NCHRP Project 20-60 was initiated in order to provide guidance and define a framework that agencies could use to identify performance measures and set performance targets. The objectives of this study were to (1) provide an assessment and recommendation for performance measures suitable for an AM approach that addresses resource allocation for transportation facility preservation, operation, improvement, and expansion; and (2) to develop a framework that decision-makers can use for selecting performance measures and setting performance targets (Cambridge Systematics 2006). This report contains two volumes. Volume I summarizes the results of the research and describes the recommended framework. It discusses the state of the current practice, criteria for performance measures that are useful for AM, considerations in designing and using performance measures and setting performance targets, and outlines a specific framework for identifying, designing, and using performance measures for AM. The performance measures framework is made up of three parts: (1) identify performance measures, (2) integrate performance measures into the organization, and (3) establish performance targets (Cambridge Systematics 2006). Volume II of this report is a guide to implementing this framework.

One of the most recent reports on AM came from FHWA in 2007. The "Asset Management Overview" (FHWA 2007a) builds upon the AM research that has been done since the publication of the "Asset Management Primer" in 1999. It examines the 
challenges to AM implementation, strategies for implementation, and the next steps for State DOTs. It also provides a summary of some of the current practices in transportation AM. With the publication of the "Asset Management Overview" along with multiple other research studies, AASHTO and FHWA have shown that they have made transportation AM a national priority. "They are providing national leadership and guidance to States as they work to incorporate asset management principles and practices into their business processes" (FHWA 2007a).

\subsubsection{State Initiatives:}

Federal agencies such as FHWA and AASHTO have produced numerous reports to assist state agencies with implementing AM. Many states have taken these principles and used the given frameworks to help begin the AM process within their own agencies. Some state agencies are using these guidelines as well as taking their own initiatives to implement AM. FHWA has published a series of comprehensive transportation AM case studies that review current practices in several states. Three comprehensive studies include: Washington State, Ohio, and North Carolina.

The case study by FHWA entitled "The Washington State Experience" (FHWA 2007d) outlines the steps that the State of Washington has taken in AM. The Washington State Department of Transportation (WSDOT) for many years had one of the key components of AM: data collection. However, even though these data collection procedures were underway during the 1980s, there was little correlation between analysis and programming. One of the driving factors to implement AM in WSDOT was the need to improve its credibility of managing the State's transportation assets. Effective 
communication with the state's leaders was necessary to prove that it was managing the state's assets properly.

In 2000, the agency worked toward moving to a GIS-based system for assets. The GIS Workbench was created so that a linear reference system would be established to “provide a crosswalk between databases" (FHWA 2007d). The department's long-term goal is to refine the Workbench so that all of the information collected can be accessed from anywhere in the department. Another technological tool that is being used for AM is the use of automated data collection vehicles. WSDOT is one of a few states to implement an automated pavement condition vehicle on $100 \%$ of the surveyed lane (FHWA 2007d). This allows for high resolution images to be examined by trained technicians in order to assess pavement conditions. From a policy standpoint, WSDOT has also created a performance report, "The Gray Notebook," to gather performance measures and benchmarks and report its progress to legislators. "The Gray Notebook" allows WSDOT to link performance measures to the department's strategic objectives.

WSDOT has made great strides in implementing a total AM system. Their efforts to increase communications have resulted in a funding increase in 2003 and again in 2005. Also, during the 2003-2005 biennium, "WSDOT moved from an allocation- to a needs-based system" (FHWA 2007d).

The FHWA case study "The Ohio Experience" (FHWA 2007c) reports that during the 1990s, the Ohio Department of Transportation (Ohio DOT) realized that it needed to restructure its organization if it wanted to maintain its capital budget. This was one of the driving factors behind implementing AM at the Ohio DOT. They knew that they needed to 1) decentralize the department and 2) develop a more accurate 
transportation asset management system that would prepare the department for the $21^{\text {st }}$ century (FHWA 2007c). As a part of restructuring, each district became responsible for its own budget so that it could better respond to individual needs within that district. One of the Ohio DOT's new policy statements made a commitment to focus on system preservation first and then consider capacity expansion only after the other bills were paid (FHWA 2007c). One of the biggest steps towards implementing a total AM system at the Ohio DOT was the development of the Base Transportation Referencing System (BTRS). The BTRS is a GIS-based program that consolidates the department's different referencing systems. This provides a common referencing system for pavements, bridges, and safety as well as project development and road inventory (FHWA 2007c).

The Ohio DOT's success in implementing an AM system can be contributed to developing a performance-based managerial system. The agency has set numerous performance targets such as a FY 2008 statewide goal for "acceptable pavement conditions on the Priority, General, and Urban systems at 90 percent" (FHWA 2007c) with similar goals for bridge conditions. Performance measures have required that the agency remain accountable in all areas of operation.

“The North Carolina Experience, Part One" (FHWA 2007b) reports that the North Carolina Department of Transportation (NCDOT) began its shift toward AM as it began to focus more on conditions and maintenance and eventually system preservation and AM. In order begin the AM process, NCDOT knew that it needed to " 1 ) obtain the best system data possible, and 2) develop a comprehensive asset management/long range plan that was performance driven" (FHWA 2007b). One of the first steps toward an AM system was the development of a methodology for categorizing infrastructure concerns. 
The first method created statewide, regional, and sub-regional classes. The second method categorized needs according to maintenance, system preservation, modernization, and expansion. As NCDOT recognized the importance of AM for the entire department, the Asset Management Office was established. This office helped support the division offices in AM activities. As the focus on AM continued, the development of management systems such as pavement (PMS), maintenance (MMS), traffic signal maintenance, bridge (BMS) and geographic information systems (GIS) began to spring up. "NCDOT's goal is for all of these systems to one day communicate with a common data system" (FHWA 2007b).

Since NCDOT began to implement AM, it has learned several lessons. Lacy Love, the AM Director, states that a DOT should start with something it already has (such as pavement management) and expand over time. Also, buy-in at all levels is critical for the success of AM. The involvement of everyone from the chief executive to the front-line manager is necessary. The third lesson is that a "DOT needs to give field personnel the tools they need in order to make intelligent decisions regarding system conditions and maintenance priorities" (FHWA 2007b).

The "Best Practices in Transportation Asset Management" (Cambridge Systematics 2007) produced by FHWA represents one of the most recent reviews of AM practices in the U.S. Its purpose is to "identify best case examples of the application of asset management principles and practice in U.S. transportation agencies" (Cambridge Systematics 2007). It not only examined six state transportation agencies, but also a city transportation department, two metropolitan planning organizations, two county transportation departments, a toll way authority, and two statewide asset management 
associations. Having a variety of transportation agencies that participated in this study allowed for a large spectrum of AM expertise to contribute to the list of best practices. The following is the observations and conclusions found as a result of this study (Cambridge Systematics 2007):

- The agencies visited had all adopted a 'preservation first' strategy for their investment priorities.

- In each case, the success of the asset management process was directly linked to the actions of an asset management champion or champions.

- In several cases, the existence of an asset management process, and more importantly, of the information that justified investment in a road system, was instrumental in securing additional dollars from the legislature.

- The most successful asset management processes have moved away from a "worst first" investment strategy, and instead have adopted investment principles that are based on life-cycle costing that result in the most costeffective preservation and maintenance strategies.

- The most successful asset management processes had performance measures that guided investment decisions throughout the organization.

- Scenario analysis showing the consequences on performance measures was one of the most effective methods of convincing decision-makers of the need for investment in the transportation system.

- There was no one organizational model for asset management. 
- The "growing pains" of an asset management process in almost all cases, fostered enhanced communications among many different organizational units.

- One of the most important starting points for implementing an asset management process is to conduct an organizational self assessment.

- There was very little evidence of the application of risk analysis techniques in the asset management processes observed.

- In several cases, agencies viewed data as an asset and the data collection process as an important decision support function.

- A customer orientation had been adopted as part of the asset management process in several cases.

- New technologies have the potential of making data collection for asset management activities more cost-effective and efficient.

- It is essential that an agency have its own performance measures/criteria documented whether they are performing maintenance activities in-house or through a private contract. 


\section{CHAPTER III}

\section{ASSET MANAGEMENT INDICATORS}

\subsection{Introduction:}

One of the major assumptions that this research rests upon is that there is an ideal AM framework that is attainable for State DOTs. This framework would contain all of the essential components of AM. For the purpose of this study, these essential components will be called AM indicators. The purpose of these indicators is to identify the successful practices of an ideal AM system for a State DOT. They reflect the most essential components of a successful AM system. In order for these indicators to exist, they need an ideal AM framework from which to be based. Some may argue that this is not possible due to the differences present in each DOT. While it is true that individual agencies may be unique in organizational structure, relationship with the legislature, and investment priorities; this does not mean that there are not common factors for AM implementation that runs through each one. The challenge is identifying a framework that is generic enough so that it can apply to every DOT, but one that has enough detail so that it has significant application.

\subsection{Asset Management Framework:}

There have been several AM frameworks created in the past several years that outline the necessary steps to implement AM. FHWA developed a framework 
that consisted of 7 components when they published the "Asset Management Primer" in 1999. The "Guide," published in 2002, also developed an AM framework. This framework has been widely accepted as a good generic framework from which to base AM for a State DOT. Instead of trying to come up with an entirely new framework, the "Guide's" framework was adopted and modified it to include an important component/indicator that this study has found essential to the AM process. Figure 3.1 represents this modified AM framework which now includes "Asset Management Culture." Each of the five framework components will serve as major indicators that point to the most successful AM practices. These major indicators each have subindicators associated with them, as can be seen from Figure 3.1. The following paragraphs will discuss the major indicators along with their associated sub-indicators.

\subsection{Asset Management Culture:}

The first component of this framework is Asset Management Culture. This indicator is one of the most difficult to define and measure, but also one of the most important. AM Culture must first be present in an agency before any of the other steps can take place.

In the summary of the "Guide", it mentions that AM is not another new program, but in fact a "way of doing business" (Cambridge Systematics 2002). A new way of doing business implies that it should affect every aspect of the agency. If AM is treated just as another program, it will not succeed. AM should be viewed as a process that integrates all of the existing management systems so that they function in unison toward a common goal instead of operating separately in the silos that traditionally exist. This 
requires a change in the mindset of those who carry out the day-to-day work activities, as well as those who are the major decision-makers within the agency. Everyone in the agency should understand that AM is a better way of doing things. This ultimately requires a change in culture. In order for AM to be successful, it must have the support of everyone involved.

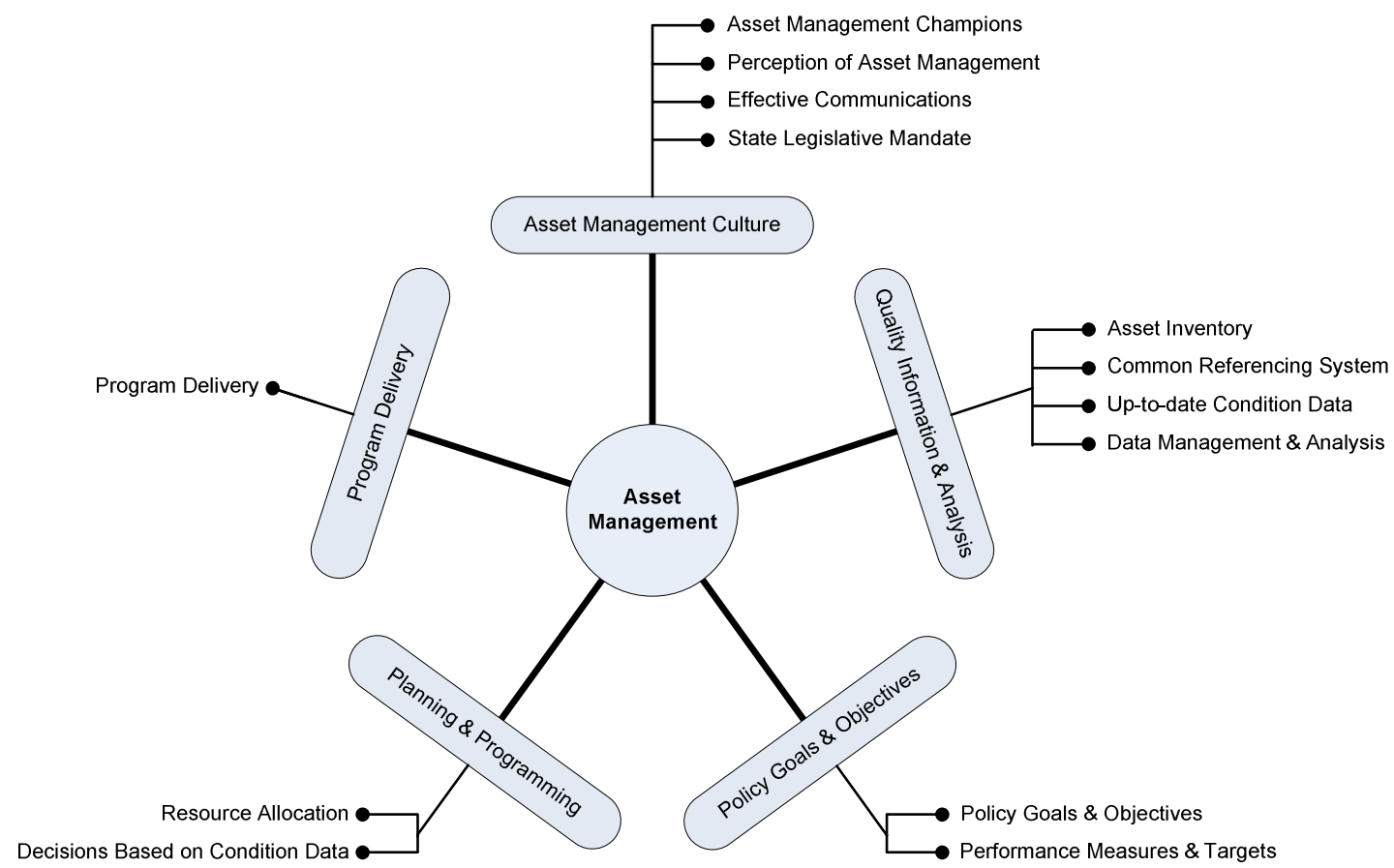

Figure 3.1: Asset Management Framework/Indicators

\subsubsection{Asset Management Champions:}

The "Best Practices in Transportation Asset Management" Scan Report reported that "the success of the asset management process was directly linked to the actions of an asset management champion or champions within the organization" (Cambridge Systematics 2007). This report stated that this champion was sometimes the head of the agency or maybe just a key staff member who believed strongly in the principles of AM. 
An AM "champion" is a person or group of persons who understand the principles of AM and are willing to be a leader in the process of implementing AM for their department. The key is that strong AM leadership must be present in order for it to be successful.

The "Guide" suggests that in order to successfully implement AM, the lead responsibility should be assigned to one individual. However, when talking to several AM leaders at State DOTs, they thought that this was not necessarily an essential element. They did, however, think that strong AM leadership from top decision-makers must be in place for AM to be successful. This leadership may come in the form of one individual, as the "Guide" suggests, or it may manifest itself in the form of several individuals that take on these responsibilities. The purpose of this study is not to specify what form leadership should be in, but just to make certain that it is present within the agency.

One way to see if an agency supports AM from top management is whether or not the director supports AM as the "way of doing business." The director does not have to necessarily be an AM champion, but he or she must understand the importance of AM and allow that practice of AM to influence the policies of the agency. Once again, several of the DOTs interviewed for this study agreed that the DOT director should support AM.

\subsubsection{Perception of Asset Management:}

The way AM is viewed within an agency is extremely important to its success. "Buy-in from all units of the agency is critical to a successful asset management effort" (Cambridge Systematics 2002). Not only must top management understand and buy into AM, but all staff should understand these principles as well. "Creating buy-in at both the 
executive and operations levels of the organization is critical to success" (FHWA 2007a). If everyone in the agency understands why AM is important and how it effects day-today decisions, it makes AM that much easier to implement.

AM is a philosophy rather than a checklist of items to be completed. Staff should be careful not to misunderstand AM as a separate, new, or competing business process (Cambridge Systematics 2002). If this happens, it would be easy for an agency to have a number of AM components in place but not use them to influence their decision-making process. This is why AM requires a change in culture in order to succeed. It requires more than a change in technical procedures, but "a transformation in agency culture based upon a change in philosophy about institutional objectives, the measurement of success, and how agency units relate to one another" (Cambridge Systematics 2002).

\subsubsection{Effective Communication:}

Effective communication is critical to the success of AM. It must be present between an agency and its governing bodies, its stakeholders and its customers (Cambridge Systematics 2002). This is important because of the change that AM brings to the transportation industry. Change naturally causes people to question why the new way is better than the old. In order for them to accept change, they must understand what is being done and why it is happening. In an agency structure, because AM must first be embraced by top management, it is important that they effectively communicate the AM vision to the staff.

Strong "vertical" communication that allows staff to understand the vision of the top management must be present within the agency. While meeting with the Utah 
Department of Transportation (UDOT) AM officials, they expressed that communication with individual regions is necessary, especially in a decentralized system such as theirs. These meetings take place twice a year and allow AM officials at UDOT's headquarters to discuss AM goals as well as performance measures (Tim Rose, personal communication, Sept. 4, 2008). This type of communication keeps regions or districts informed of the goals and objectives of the department while also allowing regions to express specific problems they are facing at the implementation level.

Strong "horizontal" communication between divisions within the agency is also necessary for AM success. "The Best Practices in Transportation Asset Management" found that agencies which have already begun to implement AM agreed that the "growing pains" of AM fostered enhanced communications among different organizational units. These same agencies recognized the need for cross organizational coordination in order to create more effective planning and decision-making (Cambridge Systematics 2007). This enhanced horizontal communication not only increases the "buyin" throughout the department, but it also reduces competition between organizational units and establishes the basis for tradeoff analysis.

\subsubsection{State Legislative Mandate:}

Several agencies, including the Michigan Department of Transportation (MDOT), have found that the existence of state legislation requiring $\mathrm{AM}$ is a strong catalyst to adopt AM principles (Cambridge Systematics 2007). In some cases this may force agencies, which may be initially uncommitted to AM, to develop an AM perspective, or at least serve as a means to begin AM within the organization. However, legislation may 
not always have to force agencies to implement AM as in the case of the Ohio DOT. They took a proactive approach and helped create AM legislation that requires a business plan to be filed every two years which reports on AM initiatives as well as key performance indicators.

\subsection{Quality Information and Analysis:}

The Virginia Department of Transportation (VDOT), as a result of initiating their Inventory and Condition Assessment System (ICAS) project, determined a new policy which stated that a credible and useful transportation inventory and condition assessment must be established and maintained (Larson and Skrypczuk 1996). Quality information is the backbone to any AM system. Without it, AM cannot function; it becomes meaningless. Data is necessary for agency objectives, the decision-making process, project delivery, and to monitor progress toward these agency objectives. Data affects every step in the AM framework. In order for an agency to be able to implement AM effectively, it must first know what assets it has, where these assets are located, and what condition the assets are in as well as be able to perform the necessary decision-supporting analysis.

\subsubsection{Asset Inventory:}

A complete and accurate asset inventory is needed (Larson and Skrypczuk 1996); otherwise system-wide analysis is meaningless. Many times, the collection of quality data is one of the first steps that an agency takes in order to successfully implement AM. When the Oregon Department of Transportation (Oregon DOT) began to implement AM, 
one of the first steps taken was to locate linear assets and begin data collection for these assets (Mark Wills, personal communication, Sept. 5, 2008). Before any true asset management can take place an agency must first know what assets it has to manage.

A central relational database that is used to store information for different classes of assets is helpful when attempting to integrate data. The Minnesota Department of Transportation (Mn/DOT) has a Transportation Information System (TIS) that acts as the central repository for data on the public road network. The TIS contains a number of subsystems that include data on bridge conditions, pavement management, and roadway history, for example (Cambridge Systematics 2007). Systems such as these do not require that data from different assets be contained in the same database, but just that this information can be accessed from a central database. This allows staff and decisionmakers to be able to access data from different asset classes without having to scan individual databases one at a time.

\subsubsection{Common Referencing System:}

It is imperative that accurate locations for all major assets exist through the use of a common spatial referencing system. Having assets tagged with spatial information allows the ability to integrate data from different sources in a relational database (Larson and Skrypczuk 1996). If assets are identified but not located in a common referencing system, it becomes very difficult to integrate data from different assets as well as determine exactly where preservation, operation, or capacity projects should be performed. AM is about strategically managing the entire system; this is extremely difficult without a common referencing system. The Ohio DOT realized this when they 
first began to implement AM. One of the first steps they took towards AM was the development of the Base Transportation Referencing System (BTRS). This is a common referencing system for both pavements and bridges. The BTRS utilizes GIS and breaks each segment into $1 / 100^{\text {th }}$ mile, for which condition data is available. Leonard Evans, an Ohio DOT official, stated that the BTRS was one of the most important steps toward implementing AM (Leonard Evans, personal communication, August 11, 2008). The Ohio DOT realized that a common referencing system was necessary for a successful AM system and they took the initiative to create a system that works well for them.

\subsubsection{Up-to-Date Condition Data:}

"Useful and reliable data are central to a fully functioning Asset Management process" (FHWA 2001). If data is to be useful, it must be current. Data that is several years old may not be useful because it does not represent the present condition of the assets that are being examined. The "Guide" states that a benchmark for effective and efficient data collection is "complete and current asset inventory and condition data." More specifically, in the self-assessment section of the "Guide," one of the questions promotes this concept by asking state agencies if they regularly collect information of the condition of their assets (Cambridge Systematics 2002). Conducting regular condition assessments allows agencies to have the most up-to-date data available which makes their data analysis more effective and in turn enhances the decision-making process. However, the term "regular" is still somewhat ambiguous. Some agencies may interpret "regular" condition assessments as every other year or may only do what is required at the federal level. The Ohio DOT, however, performs bridge inspections and pavement assessments 
every year (Leonard Evans, personal communication, August 11, 2008). This allows an excellent database from which to perform decision-supporting analysis. The Ohio DOT feels that AM is better supported by having condition assessments every year. This may not be a possibility for some states with limited funding. More condition assessments mean more dollars from a budget that, in many cases, is already stretched thin. While collecting data every year may be the best scenario, the most important factor is that data is being collected on a regular basis so that decision-makers are comfortable with data that is reliable.

If data is to be reliable, then it must be complete and consistent. Complete data means that there is condition information for all of the assets that are being managed. This is necessary so that system-wide analysis can be performed. Consistent data is repeatable and falls in line with previously collected and succeeding data. For example, consistent condition data should demonstrate a decreasing condition rating for a pavement as time progresses. Inconsistent data may show a decrease in condition rating one year followed by an increase the next. This type of data is not reliable. UDOT found that consistent data was a major key in successfully implementing AM. As they tried to implement AM, they discovered that their data was inconsistent and therefore unreliable. This limited their ability to perform quality data analysis that is needed for successful AM (Tim Rose, personal communication, Sept. 4, 2008). Good, consistent, and complete data is essentially for AM to function properly. 


\subsubsection{Data Management and Analysis:}

Asset data should be managed within a formalized data management framework (Mark Wills, personal communication, Sept. 5, 2008). This allows data to be organized and integrated so that it can be quickly utilized by staff members to perform AM supporting analysis. UDOT agrees that data management is an important component by stating: "a computer-based data management system augmented with analysis capability is a very important enabling tool for an agency's asset management program" (Cambridge Systematics 2007).

There should also be a dedicated person(s) to managing data management and analysis software. A knowledgeable and experienced data integration leader and an expert data manager are needed to design a modular, robust, and maintainable architecture that can support the expanding and changing transportation decision-support requirements (FHWA 2001). This is an especially important point if there is a high turnover rate of personnel in this area. If there are no experts available in the area of data management then it becomes difficult to update and make readily available the analysis necessary for AM to function properly. NCHRP Project 20-57 found that the most successful analytical tool applications occurred when an agency commitment to integrating the tool within its decision-making process and supported an internal "champion" to improve the tool over time (Cambridge Systematics 2005).

Asset analysis software should be utilized to identify needs in the system. These types of decision support tools should provide an economic perspective on facility conditions and calculate performance measures across all asset types as well as predict the future outcomes of decisions under consideration. Many agencies have tools that 
possess the capability to perform such functions, but their use by management for decisions such as resource allocation and program tradeoffs is not very frequent (Cambridge Systematics 2002). Instead of using software as an asset database, its analysis capabilities should be utilized to affect decisions that support AM.

Once quality data is available, DOTs should be able to provide the capability to project future asset condition through the use of deterioration models. Ideally, these systems will use actual data to update these deterioration models (Cambridge Systematics 2002). This is where the value of many years of quality data becomes extremely important. Agencies that do not have quality data, or are just beginning to collect quality data will not be able to perform this analysis. The Ohio DOT uses data from the ten previous years' to predict future asset performance and plan future projects (Leonard Evans, personal communication, August 11, 2008). This type of analysis is extremely important to the AM process. If future asset performance can be predicted, then preventative measures such as maintenance and rehabilitation can be programmed before an asset falls into disrepair that requires reconstruction. This is a part of strategically managing assets in a successful AM system.

\subsection{Policy Goals and Objectives (Major Indicator):}

The role of Policy Goals and Objectives is to establish a clear guidance for the remaining steps in the AM framework (Cambridge Systematics 2002). This drives the decision-making process of an agency. It allows an agency to set priority investment areas which gives direction and makes it easier to set objectives. The purpose of AM is to strategically manage assets with a long-term plan in mind. It is to manage the system as a 
whole working towards a common goal instead of different investment areas competing against one another for funding. Policy goals help give the agency this strategic vision that enables the agency to embrace AM.

\subsubsection{Policy Goals and Objectives (Sub-Indicator):}

It is important that an agency select a priority investment area. "The asset management framework does not prescribe what priorities should come first - only that individual agencies and their policy-making bodies discuss and analyze policy options to adopt the ones that are felt to be warranted" (Cambridge Systematics 2002). There are three major investment areas that a DOT may choose to place a top priority. Typically, these three areas are: system preservation, transportation system management and operations, and capacity expansion. Each of these areas has its own individual merits and is important to the transportation system.

System preservation is the top priority for many agencies because of the current condition of highway systems in the United States. Mature states face this issue as most of their highway systems have been completed and now their primary focus has shifted towards maintenance and rehabilitation. The Ohio DOT made the commitment to budget for system preservation first and then address all other needs after that point. The Florida Department of Transportation (FDOT) also considers system preservation to be very important, evidenced by the fact that they fund maintenance needs before any capacity expansion projects are considered (Cambridge Systematics 2007). However, other states such as Utah are still growing which requires capacity expansion to be a priority (Tim Rose, personal communication, Sept. 4, 2008). Others may consider placing systems 
operations as a priority as they receive pressure to adopt "reduce congestion" strategies (Cambridge Systematics 2007). AM does designate which of these priorities should be placed first, rather it should be up to individual agencies to analyze their situation and decide which one best fits their needs and should be placed ahead of the others.

Policy goals and objectives should guide the agency's overall decision process. The Ohio DOT's decisions are guided by a set of principles and strategic goals that are linked to performance measures that are found at all levels of organizational decisionmaking (Cambridge Systematics 2007). Based on this, it is clear that there should be a plan with goals and objectives, but this plan must directly affect decision-making to truly be effective. Policies that do not influence decision-making can often be vague and therefore difficult to identify. This is why policies should lead directly to action by pointing to specific, quantifiable, and achievable targets.

Policy goals and objectives should support a long term life-cycle approach to managing assets rather than traditional "worst first" approaches. "Most successful asset management processes have moved away from a 'worst first' investment strategy, and instead have adopted investment principles that are based on life-cycle costing" (Cambridge Systematics 2007). Traditionally, many states plan and program their projects on a "worst first" basis. Because DOT budgets only cover time horizons of 1 to 2 years, committing funds for the long term is very difficult. This, combined with the uncertainty of future funds, places pressure to select the projects with the lowest initial cost rather than looking at the total life-cycle cost (FHWA 1999). AM should be about making cost-effective decisions; ones that benefit the system as a whole. This fits into 
strategically managing assets. AM supports life-cycle costing because of its long-term view of performance and cost (Cambridge Systematics 2002).

Policies should be related to objectives, performance measures, and performance targets from the very beginning. As policy goals are developed, performance measures should be defined at the same time (Cambridge Systematics 2002). This gives agencies something to measure against. It is a type of feedback that allows decision-makers to gauge how well policies are enabling the agency to meet specified targets. If targets are not being met, then perhaps policies need to be evaluated to determine if they need to be improved.

\subsubsection{Performance Measures \& Targets:}

Performance measures provide the critical link between policy goals and planning and programming decisions (Cambridge Systematics 2002). They allow agencies to measure what affect policy decisions have on programming. They are a way to monitor progress toward a result or goal and are indicators of work performed and the results achieved (Cambridge Systematics 2006). Because of their critical role, it is essential that agencies have performance measures and targets present in their organization. An example would be what measures FDOT has for its pavements and bridges. Their objective is that $80 \%$ of state highway pavements meet the department's standards (6 out of 10 condition rating). The objective for bridges is that $90 \%$ of bridges meet standards which are defined as not showing evidence of structural deterioration, not being limited by weight restrictions or not needing preventative maintenance (Cambridge Systematics 2007). 
However, simply having performance measures in place for pavements and bridges does not ensure that the agency is on its way to successfully implementing AM. It is vital that an agency use performance measures to govern their decision-making process. Policy goals and the associated performance measures and targets should guide an agency's overall resource allocation and program delivery (Cambridge Systematics 2006). Mn/DOT states that once they switched to a performance management approach, it was no longer a matter of which projects should be a part of the investment program, but how the performance targets could be reached (Cambridge Systematics 2007).

The amount of resources necessary to accomplish specified performance targets should be known. Policy objectives and targets should only be set after first analyzing the costs to achieve different levels of condition and performance. This will enable an agency to establish realistic targets (i.e., targets that are achievable given existing funding restraints, traffic usage, etc.) (Cambridge Systematics 2002). It is possible that an agency, with good intentions, may set goals that are idealistic and therefore not achievable. An agency should set targets that can be reached within their own restrictions and circumstances; otherwise, they may mislead stakeholders and users as to what their capabilities are. For example, the Ohio DOT knows that for $\$ \mathrm{X}, \mathrm{Y}$ amount of maintenance can be performed. For 10 percent more money, 15 percent more maintenance can be performed (Cambridge Systematics 2006). Being able to present data such as this to stakeholders will show that an agency is knowledgeable on what specific goals it can accomplish given a certain amount of money. This may lead to being able to secure additional funding. Maximizing performance targets should be the goal of every agency wishing to implement AM. 
Once performance targets are set according to what the agency can accomplish, user feedback should also be considered to determine what is acceptable. "The selected measures should be able to reflect customer perceptions of system performance and quality of service where appropriate" (Cambridge Systematics 2002). One concern may be that performance targets are set lower than customer expectations. If this is the case, targets must be raised until they reach a satisfactory level. However, an agency may set targets that are higher than customer expectations. While agency officials may see the need to set targets this high, ultimately it is unnecessary and an unwise use of resources. "Only by regularly collecting customer perceptions of asset condition and performance, as well as their expectations, can agencies maintain or increase customer satisfaction" (FHWA 2007a). Targets should be set to meet customer expectations of asset performance.

\subsection{Planning and Programming:}

Planning and programming is important because in this step decisions are made about what projects should be selected. Once goals and objectives along with performance measures have been established, decision-makers can now perform tradeoff analysis and allocate resources across the network as needed. Overall, planning and programming allows policy goals and objectives to be realized in a practical way through the decision-making process. 


\subsubsection{Resource Allocation:}

Asset Management "involves applying general principles smartly, effectively, and tactically to resource allocation and utilization - the heart of asset management" (Cambridge Systematics 2002). Resource allocation is important because it represents the direct application of AM principles. Policies and goals do not mean anything unless they directly affect decision-making procedures. Resource allocation is a decision-making process that should be affected by AM policies. This process should be comprehensive, viewing the transportation system as an integrated whole which considers tradeoffs among investment areas (Cambridge Systematics 2002). This means that tradeoff analysis across all major assets should be performed when making resource allocation decisions. This facilitates examining the system as a whole and making resource allocation decisions that benefit the system instead of favoring a certain asset type or region over another.

Resources should also be able to be moved across districts and assets based upon need. The Ohio DOT allocates a certain amount of money to each district which then has the ability to spend that money in the most efficient manner. Each district can examine its needs and determine what should be spent on bridges, pavements, etc. They also have the ability to re-allocate funds from one district to another so that the needs of the entire system are best met (Leonard Evans, personal communication, August 11, 2008). By having the capability to move resources regardless of district or asset means that an agency is committed to doing what is best for the entire system instead of keeping funds in silos that traditionally exist. 


\subsubsection{Decisions Based On Condition Data:}

Resource allocation decisions should be made across programs and geographic regions based on expected performance rather than historical splits or formulas that do not correlate with an objective indication of system condition (Cambridge Systematics 2002). The Ohio DOT's districts' budgets are driven by the condition of the assets for which they are responsible (Cambridge Systematics 2007). This is how quality condition data should support the decision-making process. If resources are allocated not according to condition data, the decision-making process is not performance-based. However, AM is performance driven and based on quality information. Both of these factors support resource allocation decisions based on condition data.

Future projects should be programmed on a regular basis according to performance forecasts which are based on condition data. The Ohio DOT not only uses past condition data to predict future performance of assets, but also to plan future projects (Leonard Evans, personal communication, August 11, 2008). Future projects of this nature may consist of preventative maintenance, rehabilitation, or reconstruction projects. Performance forecasts based on past condition data will allow agencies the capability to predict failures within their system and then program the appropriate project to fix that failure. The ability to program projects and deliver them in a timely manner is more efficient than waiting for an asset to deteriorate before programming its repair. This may allow a less expensive repair to be completed instead for performing reconstruction, for example. "State DOTs report that the proactive approach of preventative maintenance known as pavement preservation - cuts the need for costly, time-consuming rehabilitation 
and reconstruction projects" (FHWA 2007a). AM is about performing the right fix at the right time (Gil Chesbro, personal communication, August 12, 2008).

\subsection{Program Delivery (Major Indicator):}

Program delivery is one of the last steps in the AM process. It is unique because it occurs after most of the "traditional" AM decisions have been made. At this point, policies and performance measures have been established, resources have been allocated, and specific projects have been programmed. However, AM also extends into this final step by making certain agencies consider the most efficient way to deliver projects. AM is strategic in nature and looks to find the best way of doing any task. Evaluating different program delivery methods may provide opportunities for reduced costs or schedules while staying within the project scope. Depending on the situation, the budget or schedule may govern the program delivery method. The purpose of program delivery in $\mathrm{AM}$ is to select the most efficient and effective way to deliver a project to the customer.

\subsubsection{Program Delivery (Sub-Indicator):}

Alternative delivery methods should be regularly evaluated within the agency. This involves an assessment of options while considering relative costs, benefits and risks, both immediate and long term (Cambridge Systematics 2002). One example of an alternative delivery method is the design-build (DB) delivery method. Traditionally many states use the design-bid-build (DBB) delivery method for their projects. DBB is often preferred because its strength is delivering projects when the budget is the primary 
concern. However, some projects may require that the schedule be primary. If this is the case, the DB option (which is schedule driven) may be a better delivery method. UDOT used DB while reconstructing 16 miles of I-15 in time for the 2002 Winter Olympics. It was estimated that if the traditional DBB method was used, the project would have taken eight to ten years to complete. However, the DB method was employed and the project was completed in five and one-half years (Cambridge Systematics 2002).

Another type of delivery method that may need to be considered by an agency is public-private partnerships (PPP). This is a contractual agreement between public and private sector partners, which usually allow a governmental agency to contract with a private company to renovate, construct, operate, maintain, and/or manage a facility or system (FHWA 2004). PPP's are sought as alternative delivery systems to address the shortfalls in funding (Aziz 2007). This is especially important since many agencies face revenues that are increasingly less than the investment requirements. While PPP's are more popular in Europe and Asia, they are also being used in the United States. Between 1985 and 2004, $\$ 42.1$ billion worth of road projects were planned and funded in the United States using PPPs (AECOM Consult 2005). This represents a fairly significant amount of funding for road projects in the United States. AM certainly does not require that PPP projects be used, but as part of evaluating and effective and economical choices, PPP's need to be considered.

As a part of program delivery, performance-based contracts need to be regularly evaluated. Performance-based contracts focus on the results, output, or outcome of the end product, not on the materials and methods used to build and maintain the product (Aziz 2007). AM calls for system performance to govern decisions throughout the project 
life cycle. State DOTs can incorporate these performance-based concepts through the use practices such as: performance specifications, cost plus time bidding ( $\mathrm{A}+\mathrm{B}$ bidding), best-value bidding (quality based selection), lane rental, life-cycle cost bidding, incentive contract clauses, and warranty periods (Cambridge Systematics 2002). Each of these methods is an innovative approach that encourages improvement in product quality or the reduction of schedule and/or budget. These objectives fit within the AM framework.

Additional opportunities in program delivery exist in outsourcing maintenance and operations activities, which should be regularly evaluated. Potential benefits of outsourcing include lower overall costs, improved service, opportunities to utilize the expertise of private companies, and overcome in-house staffing constraints (Cambridge Systematics 2002). Some agencies outsource data collection. When UDOT decided to switch to automated data collection in order to maintain consistent data, they outsourced their data collection to a private company (Tim Rose, personal communication, Sept. 4, 2008). This demonstrates taking advantage of the expertise of a private entity. According the "Best Practices in Transportation Asset Management," several agencies use private contractors to perform long-term maintenance services while others still primarily relied on their own forces (Cambridge Systematics 2007). This illustrates that it is not important which maintenance method an agency uses in order to successfully implement AM, but rather that an agency evaluate outsourcing options and choose the best one based on their current situation. 


\section{CHAPTER IV}

\section{ASSESSMENT OF ASSET MANAGEMENT INDICATORS}

\subsection{Introduction:}

As a result of the previous chapter, the necessary AM indicators have now been identified. However, simply the identification of critical factors for AM is nothing new to research in this field. FHWA developed a list of components for an AM program with the publication of the “Asset Management Primer” in 1999 (FHWA 1999). This represented one of the earliest efforts in transportation AM. Even in the early stages AM, a basic set of factors were identified. Several years later the "Guide" (Cambridge Systematics 2002) established an AM framework that is now universally accepted among transportation agencies and officials. The "Guide" also provides a self-assessment section that allows agencies to assess their current status of AM. The objective of this study is very closely related to the self-assessment section in the "Guide." However, there is one major difference between this research and the self-assessment tool. The self-assessment tool assumes that each component and each factor of AM is of equal importance. This is indicated by the absence of weights when computing one's score at the end of the section. By its own definition, the self-assessment is meant to be a quick diagnostic tool that gives an overall impression, not a detailed measure of where an agency stands with respect to AM. The "Guide" states that the results of the self-assessment are specific to an “agency's management environment and financial, organizational, institutional, and 
technological situations" and therefore do not provide a meaningful basis for comparisons with peer agencies (Cambridge Systematics 2002). This, along with the absence of weighting factors is how this study differs from the self-assessment tool.

One assumption that this study rests upon is that there is an ideal AM system available to State DOTs. This ideal system is generic enough so that it can be applied to every DOT, but still specific enough so that it has meaningful application. Another assumption is that this system has components that are more important than others when implementing an ideal AM system. If this is true, then these components or indicators need to be weighed according to their relative importance. This has not been done in previous research. Several different AM frameworks with many common components have been developed, but these components have never been weighed based on their importance in implementing an ideal AM system. If accurate weights for these indicators can be developed, a more precise assessment tool could then be presented. The question then arises as to how to quantity the level of importance of the AM indicators. To achieve this, a suitable methodology must be chosen.

\subsection{The Analytic Hierarchy Process:}

The Analytic Hierarchy Process (AHP) is a methodology used in multicriteria decision making that was introduced by Saaty (1980). It is a theory of measurement that is used to quantify qualitative or subjective factors that affect a decision. AHP is applied by first structuring a hierarchy. The top level in the hierarchy is the goal. The subsequent levels that fall beneath the goal are criteria and subcriteria, respectively. There can be several levels of subcriteria. "The purpose of the structure is to make it possible to judge 
the importance of the elements in a given level with respect to some or all of the elements in the adjacent level above" (Saaty and Vargas 2001). This is carried out using pairwise comparisons which form a matrix for each level in the hierarchy. The weights (or level of importance) for each subcriteria and criteria are obtained by computing the principal eigenvector, normalized to become the vector of priorities. These are the weights for the factors within each level. To obtain an overall weight for each subcriterion, the weight of an individual subcriterion is multiplied by the weight of its associated criterion in the level above it.

AHP was chosen to quantity the AM indicators because of its well-known and widespread application as well as its relative ease of use. The chosen methodology should be able to take the subjective opinions of AM experts and convert them into quantifiable numbers that weigh the importance of the AM indicators. For this purpose, AHP is an excellent method that can fulfill these requirements.

\subsection{Application of the Analytic Hierarchy Process:}

The most effective way to apply AHP to a broad number of participants was through the use of a questionnaire survey. This survey allowed the research team to collect the opinions of AM experts concerning the relative importance of the AM indicators. It permitted AM experts to assess the AM indicators and converted their opinions into quantifiable weights. 


\subsubsection{Survey Design:}

A questionnaire survey was developed to assess the importance of each AM indicator. This survey can be found in Appendix A. The first section of the survey consists of the definitions of the AM indicators, as defined by the research team. These indicators represent the components necessary to successfully implement an ideal AM system at a State DOT. The word "ideal" is stressed several times throughout the survey so that the respondents will be aware that they are not evaluating components necessary for their particular DOT, but what is necessary for an ideal system. These definitions were needed so that all respondents would assess the AM indicators based on common definitions.

The second section of the survey explains the methodology that is used to assess the AM indicators. This method is an application of AHP. AHP works by comparing indicators two at a time. In order to do this, a numerical scale is needed to designate the dominance of one indicator over another. Saaty (1980) developed such a scale that consists of numbers $1,3,5,7,9$ in which 1 represents equal importance and 9 represents extreme importance over another indicator. This scale is shown in Table 4.1. There are also reciprocal values in this scale. If indicator $i$ has one of the numbers assigned to it when compared with indicator $j$, then $j$ has the reciprocal value when compared with $i$ (Saaty and Vargas 2001). A very similar table, slightly modified to compliment the needs of the survey, is present in the second section. To ensure that the respondents will apply the pairwise comparisons in the correct order, an example of how to apply these comparisons is given at the bottom of the second section. 
Table 4.1: The Fundamental Scale (Saaty and Vargas 2001)

\begin{tabular}{|c|c|c|}
\hline Intensity of Importance & Definition & Explanation \\
\hline 1 & Equal Importance & $\begin{array}{l}\text { Two activities contribute } \\
\text { equally to the objective }\end{array}$ \\
\hline 3 & Moderate Importance & $\begin{array}{l}\text { Experience and judgment } \\
\text { slightly favor one activity } \\
\text { over another }\end{array}$ \\
\hline 5 & Strong Importance & $\begin{array}{c}\text { Experience and judgment } \\
\text { strongly favor one activity } \\
\text { over another }\end{array}$ \\
\hline 7 & $\begin{array}{c}\text { Very Strong or } \\
\text { demonstrated importance }\end{array}$ & $\begin{array}{c}\text { An activity is favored very } \\
\text { strongly over another; its } \\
\text { dominance demonstrated in } \\
\text { practice } \\
\end{array}$ \\
\hline 9 & Extreme Importance & $\begin{array}{l}\text { The evidence favoring one } \\
\text { activity over another is of } \\
\text { the highest possible order of } \\
\text { affirmation }\end{array}$ \\
\hline
\end{tabular}

The third section of the survey consists of applying AHP to compare the five major indicators. This is done through a series of pairwise comparisons in order to determine which indicator is the most important when implementing an ideal AM system. The five major indicators are:

- Asset Management Culture

- Quality Information \& Analysis

- Policy Goals \& Objectives

- Planning \& Programming

- Program Delivery

The fourth section is similar to the third except that it consists of comparing the sub-indicators through a series of pairwise comparisons. Each set of sub-indicators falls 
beneath one of the five major indicators. The sub-indicators are listed beneath their associated major indicator:

- Asset Management Culture

- Asset Management Champions

○ Perception of Asset Management

- Effective Communication

- State Legislative Mandate

- Quality Information \& Analysis

- Asset Inventory

○ Common Referencing System

○ Up-to-Date Condition Data

○ Data Management \& Analysis

- Policy Goals \& Objectives

○ Policy Goals \& Objectives

○ Performance Measures \& Targets

- Planning \& Programming

○ Resource Allocation

- Decisions Based on Condition Data

- Program Delivery

○ Program Delivery

The fifth section of the survey collects general information about the respondent. This provides the research team with the respondent's name, transportation agency, years of experience in transportation as well as AM, and the respondent's position/title. This 
information allows the research team to validate the respondent's credibility based on the transportation agency for which they are employed as well as the number of years of experience in the transportation industry.

\subsubsection{Survey Participants:}

It was determined that, in order to have meaningful results, a random survey would not meet the needs for this research. The purpose of the survey is to collect opinions of AM experts. In using a random survey, some of the collected data would inevitably be from DOT officials that are not well-versed in total AM systems. Every State DOT is at a different stage of implementing AM, while some have not begun to implement AM at all. Information collected from DOT officials that are not familiar with AM principles would yield less meaningful data and contribute to a model that would not be very useful. Based on this, it was determined that a selective survey would be used.

Potential survey respondents were selected from State DOTs that are known to be leaders in AM. As previously mentioned, each state that is implementing AM is at a different stage of development. Some are very advanced whereas others are just beginning the AM process. The major source of identifying DOTs that are leaders in AM came from the literature review. This included case studies and recent research that referenced DOTs that are making advances in AM. Several others states were identified by recommendations from peer agencies. Also, several surveys were sent to representatives from the Office of Asset Management at FHWA. In all, twenty-six surveys were sent to AM officials at fifteen different transportation agencies. Fourteen of these were State DOTs and one was the Office of Asset Management at FHWA. Each of 
these surveys was sent by email and regular mail to the potential respondent. Of the twenty-six surveys that were mailed, sixteen were completed and returned. This yielded a response rate of $16 / 26$ or $61.5 \%$. The sixteen returned surveys represented fourteen different transportation agencies (thirteen DOTs and FHWA). The agencies that participated in the survey were: Florida DOT, Louisiana DOTD, Maryland DOT, Minnesota DOT, New York State DOT, North Carolina DOT, Ohio DOT, Oregon DOT, Pennsylvania DOT, Utah DOT, Virginia DOT, Vermont DOT, Washington State DOT, and FHWA Office of Asset Management. These agencies are shown on a map in Figure 4.1.

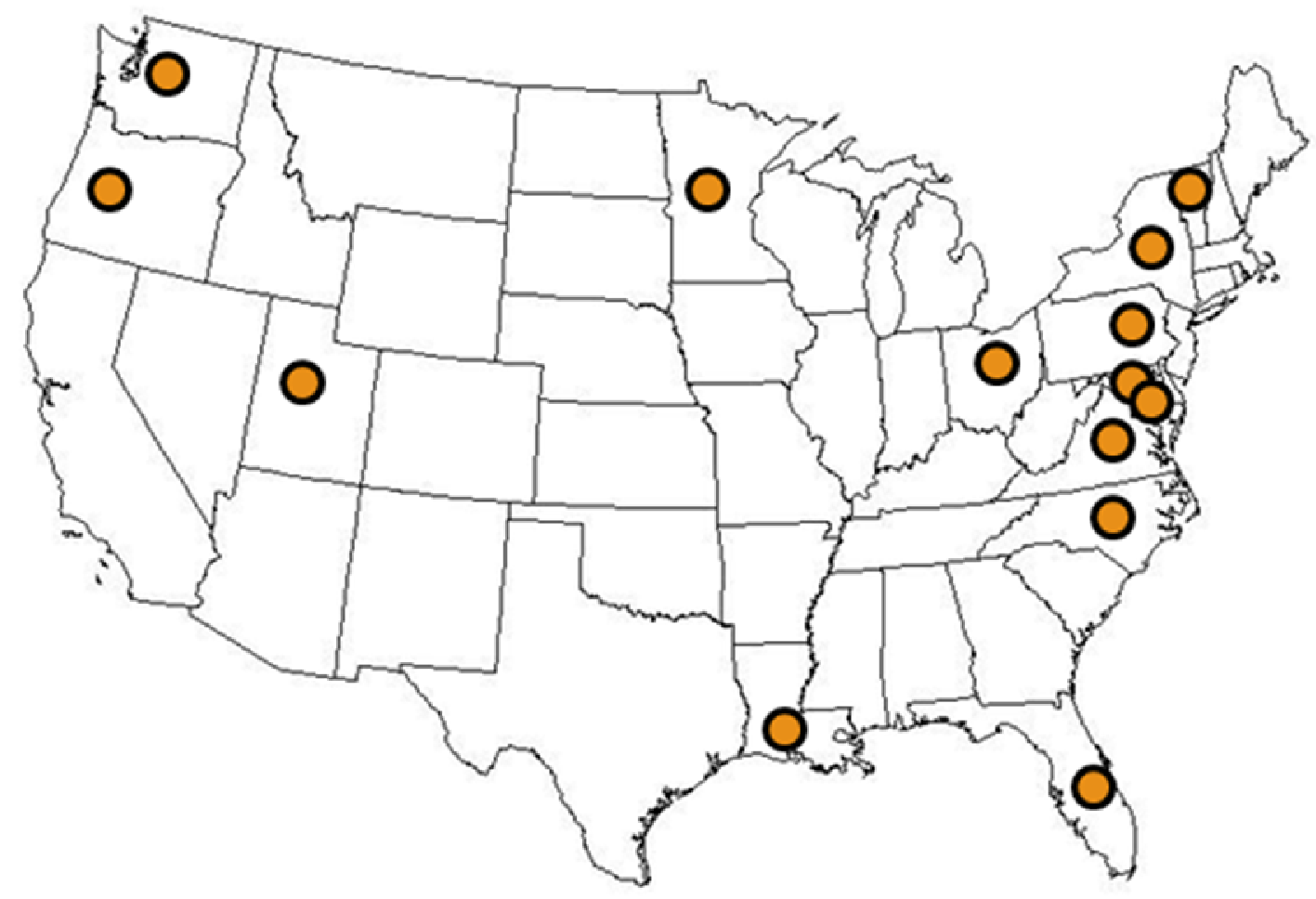

Figure 4.1: Indicator Ranking Survey Participants 
The number of years of experience in the transportation industry among the survey respondents was fairly high. The collective amount of experience was 339 years among 16 professionals which resulted in an average amount of experience of 21.2 years per person. There was only one respondent who had less than 10 years of experience in the transportation industry. The average amount of AM experience among the respondents was 6.8 years. This number is much lower than the overall transportation experience, but this is easily explained due to the fact that AM programs (as they are known today) have only been around for approximately ten years.

\subsection{Survey Results:}

Each completed survey generated five reciprocal matrices whose values were composed from the responses of that participant. Of the five matrices, there was one $5 \times 5$ matrix, two 4 x 4s, and two $2 \times 2$ matrices. For each matrix, eigenvalues and eigenvectors are calculated. An eigenvector for each indicator within a given matrix is calculated and these values are normalized to create weights for each indicator. However, calculating eigenvalues and eigenvectors is only the first step in the process. AHP has a method to measure consistency of the responses within a matrix.

\subsubsection{Consistency Ratios:}

The consistency ratio (C.R.) is a measure of how consistent a matrix is within AHP. The C.R. for each matrix is found by first calculating the consistency index (C.I.). This is represented by C.I. $=\left(\lambda_{\max }-n\right) /(n-1)$, where $\lambda_{\max }$ is equal to the largest eigenvalue and $n$ is equal to the order of the matrix. The C.R. is then calculated by taking 
the C.I. and dividing it by the corresponding value from the random index table based on the order of the matrix. Shown in Table 4.2, Saaty (1980) provides a random index table that contains the order or the matrix (first row) and the random index value (second row). The threshold value that indicates acceptable consistency is a C.R. equal to 0.10. If the C.R. is above this threshold, it must be reduced. There are several methods available to improve the consistency of the matrix.

Table 4.2: Random Index Table (Saaty 1980)

\begin{tabular}{|c|c|c|c|c|c|c|c|c|c|c|c|c|c|c|}
\hline 1 & 2 & 3 & 4 & 5 & 6 & 7 & 8 & 9 & 10 & 11 & 12 & 13 & 14 & 15 \\
\hline 0.00 & 0.00 & 0.58 & 0.90 & 1.12 & 1.24 & 1.32 & 1.41 & 1.45 & 1.49 & 1.51 & 1.48 & 1.56 & 1.57 & 1.59 \\
\hline
\end{tabular}

For each completed survey for this research, the C.R. for each matrix was calculated except for the $2 \times 2$ matrices, whose random index value is equal to 0.00 . After calculating the C.R.s for all sixteen surveys, it was found that only two surveys met the threshold limit of C.R. $=0.10$ for all of the matrices. Even though many C.R.s were not very far above 0.10 , they still required improved consistency.

The best method of improving consistency is by allowing the respondents to revise their judgments. This requires the respondents to make a conscious effort to apply their comparisons in a more consistent manner. While this is the best method of improving consistency, it is also one of the most difficult to apply. When AHP is applied through the use of a survey, as it was in this case, it is very difficult to ask the respondents to revise their judgments. Each AM expert would most likely not have time to re-examine a tedious survey to try to identify inconsistencies in their original judgments. Based on this argument, another acceptable method of judgmental revision was employed. 
This research used a method that Saaty (1980) suggests to improve consistency which revises the original judgments in an "artificial" manner. Consider the $3 \times 3$ matrix given in Figure 4.2. The first step to revise a matrix is to form a matrix of priority ratios $w_{i} / w_{j}$ (Figure 4.3) and consider the matrix of absolute differences $\left[\left|a_{i j}-\left(w_{i} / w_{j}\right)\right|\right]$ (Figure 4.4). The row with the largest sum should be selected and all of the $a_{i j}$ in the row in question should be replaced with the $w_{i} / w_{j}$ values for that row. Once this is completed, a new C.R. is calculated. If the C.R. is still greater than 0.10, this procedure is repeated with the new matrix. Repetition of this process has been noted to improve consistency, however, Saaty (1980) cautions against excessive use of this process of forcing the values of judgments to improve consistency.

$$
\left(\begin{array}{lll}
a_{11} & a_{12} & a_{13} \\
a_{21} & a_{22} & a_{23} \\
a_{31} & a_{32} & a_{33}
\end{array}\right)\left(\begin{array}{l}
w_{1} \\
w_{2} \\
w_{3}
\end{array}\right)
$$

Figure 4.2: 3 × 3 matrix

$$
\left(\begin{array}{lll}
\frac{\mathrm{w}_{1}}{\mathrm{w}_{1}} & \frac{\mathrm{w}_{1}}{\mathrm{w}_{2}} & \frac{\mathrm{w}_{1}}{\mathrm{w}_{3}} \\
\frac{\mathrm{w}_{2}}{\mathrm{w}_{1}} & \frac{\mathrm{w}_{2}}{\mathrm{w}_{2}} & \frac{\mathrm{w}_{2}}{\mathrm{w}_{3}} \\
\frac{\mathrm{w}_{3}}{\mathrm{w}_{1}} & \frac{\mathrm{w}_{3}}{\mathrm{w}_{2}} & \frac{\mathrm{w}_{3}}{\mathrm{w}_{3}}
\end{array}\right)
$$

Figure 4.3: Priority Ratios 


$$
\left(\begin{array}{lll}
\mathrm{a}_{11}-\frac{\mathrm{w}_{1}}{\mathrm{w}_{1}} & \mathrm{a}_{12}-\frac{\mathrm{w}_{1}}{\mathrm{w}_{2}} & \mathrm{a}_{13}-\frac{\mathrm{w}_{1}}{\mathrm{w}_{3}} \\
\mathrm{a}_{21}-\frac{\mathrm{w}_{2}}{\mathrm{w}_{1}} & \mathrm{a}_{22}-\frac{\mathrm{w}_{2}}{\mathrm{w}_{2}} & \mathrm{a}_{23}-\frac{\mathrm{w}_{2}}{\mathrm{w}_{3}} \\
\mathrm{a}_{31}-\frac{\mathrm{w}_{3}}{\mathrm{w}_{1}} & \mathrm{a}_{32}-\frac{\mathrm{w}_{3}}{\mathrm{w}_{2}} & \mathrm{a}_{33}-\frac{\mathrm{w}_{3}}{\mathrm{w}_{3}}
\end{array}\right)
$$

Figure 4.4: Matrix of Absolute Differences

This procedure was carried out for all of the matrices that did not meet the required C.R. $=0.10$. Out of the forty-eight matrices, ( 3 matrices for each survey, 16 surveys) twenty-eight required revisions in order to improve consistency. Twenty-two of these matrices that required improvement only needed one iteration to reduce the C.R. to 0.10 or less. Six matrices required two iterations. There were none that required more than two iterations to improve the C.R. to the acceptable level. The revised C.R.s for each matrix along with the number of iterations to achieve the acceptable level are shown in Table 4.3.

\subsubsection{Synthesis of the Data:}

Once each of the matrices has an acceptable C.R., the weights of each one can be included in the synthesis procedure to create a composite weight for each indicator. To synthesize their results from a questionnaire that used AHP, Zayed and Chang (2002) calculated the average weight and standard deviation for each factor in their model. This procedure was used for this research to create the composite weights for each indicator that was evaluated in the survey. The average weight and standard deviation for each of 
the five major indicators and each of the thirteen sub-indicators was calculated. These results are summarized in Table 4.4 and 4.5 and shown graphically Figures 4.5 and 4.6. Total weights for sub-indicators are obtained by multiplying the weight of each subindicator by the weight of its associated major indicator. This is the final objective of using AHP as a tool to assign weights to the indicators. The total weights will be directly used in the AM Assessment Model because they represent the relative importance of each sub-indicator. The total weights are summarized in Table 4.6 and shown in Figure 4.7.

Table 4.3: Revised Confidence Ratios

\begin{tabular}{|c|c|c|c|}
\hline & \multicolumn{3}{|c|}{ Matrices } \\
\hline Transportation Agency & $\begin{array}{c}\text { Major } \\
\text { Indicators }\end{array}$ & $\begin{array}{c}\text { Asset Management } \\
\text { Culture }\end{array}$ & $\begin{array}{c}\text { Quality Information } \\
\text { \& Analysis }\end{array}$ \\
\hline Florida DOT & 0.065 & 0.089 & 0.097 \\
\hline FHWA & 0.027 & 0.015 & 0.057 \\
\hline Louisiana DOTD & 0.052 & 0.043 & 0.042 \\
\hline Maryland DOT & 0.037 & 0.096 & 0.026 \\
\hline Minnesota DOT & 0.089 & 0.019 & 0.090 \\
\hline North Carolina DOT & 0.067 & 0.043 & 0.047 \\
\hline New York State DOT & 0.058 & 0.057 & 0.000 \\
\hline Ohio DOT & 0.024 & 0.050 & 0.016 \\
\hline Oregon DOT & 0.035 & 0.069 & 0.043 \\
\hline Pennsylvania DOT & 0.054 & 0.015 & 0.057 \\
\hline Utah DOT & 0.045 & 0.012 & 0.043 \\
\hline Utah DOT & 0.039 & 0.043 & 0.000 \\
\hline Utah DOT & 0.067 & 0.039 & 0.084 \\
\hline Vermont DOT & 0.024 & 0.057 & 0.043 \\
\hline Virginia DOT & 0.097 & 0.059 & 0.045 \\
\hline Washington State DOT & 0.009 & 0.000 & 0.000 \\
\hline
\end{tabular}

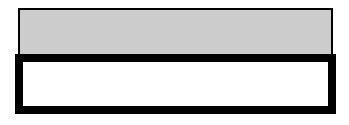

1 Iteration

2 Iterations 
Table 4.4 Major AM Indicator Weights

\begin{tabular}{|c|c|c||c|}
\hline Major Indicators & $\begin{array}{c}\text { Lower Limit } \\
\text { (-Std Dev) }\end{array}$ & Mean & $\begin{array}{c}\text { Upper Limit } \\
\text { (+Std Dev) }\end{array}$ \\
\hline $\begin{array}{c}\text { Asset Management } \\
\text { Culture }\end{array}$ & 0.067 & 0.195 & 0.322 \\
\hline $\begin{array}{c}\text { Quality Information } \\
\text { \& Analysis }\end{array}$ & 0.149 & 0.265 & 0.381 \\
\hline $\begin{array}{c}\text { Policy Goals \& } \\
\text { Objectives }\end{array}$ & 0.165 & 0.300 & 0.435 \\
\hline \hline $\begin{array}{c}\text { Planning \& } \\
\text { Programming }\end{array}$ & 0.047 & 0.123 & 0.199 \\
\hline \hline Program Delivery & 0.012 & 0.118 & 0.224 \\
\hline
\end{tabular}


Table 4.5: AM Sub-Indicator Weights

\begin{tabular}{|c|c|c|c|c|}
\hline $\begin{array}{c}\text { Major } \\
\text { Indicators }\end{array}$ & Sub-Indicators & $\begin{array}{c}\text { Lower } \\
\text { Limit } \\
\text { (-Std Dev) }\end{array}$ & Mean & $\begin{array}{c}\text { Upper } \\
\text { Limit } \\
\text { (+Std Dev) }\end{array}$ \\
\hline \multirow{4}{*}{$\begin{array}{c}\text { Asset } \\
\text { Management } \\
\text { Culture }\end{array}$} & $\begin{array}{l}\text { Asset Management } \\
\text { Champions }\end{array}$ & 0.096 & 0.272 & 0.449 \\
\hline & $\begin{array}{c}\text { Perception of Asset } \\
\text { Management }\end{array}$ & 0.092 & 0.216 & 0.339 \\
\hline & $\begin{array}{c}\text { Effective } \\
\text { Communication }\end{array}$ & 0.120 & 0.303 & 0.487 \\
\hline & $\begin{array}{c}\text { State Legislative } \\
\text { Mandate }\end{array}$ & -0.014 & 0.209 & 0.431 \\
\hline \multirow{4}{*}{$\begin{array}{c}\text { Quality } \\
\text { Information \& } \\
\text { Analysis }\end{array}$} & Asset Inventory & 0.166 & 0.311 & 0.455 \\
\hline & $\begin{array}{c}\text { Common } \\
\text { Referencing System } \\
\end{array}$ & 0.131 & 0.296 & 0.461 \\
\hline & $\begin{array}{c}\text { Up-to-Date } \\
\text { Condition Data } \\
\end{array}$ & 0.092 & 0.204 & 0.316 \\
\hline & $\begin{array}{c}\text { Data Management \& } \\
\text { Analysis } \\
\end{array}$ & 0.064 & 0.190 & 0.315 \\
\hline \multirow{2}{*}{$\begin{array}{c}\text { Policy Goals \& } \\
\text { Objectives }\end{array}$} & $\begin{array}{c}\text { Policy Goals \& } \\
\text { Objectives }\end{array}$ & 0.319 & 0.570 & 0.821 \\
\hline & $\begin{array}{c}\text { Performance } \\
\text { Measures \& Targets } \\
\end{array}$ & 0.179 & 0.430 & 0.681 \\
\hline \multirow{2}{*}{$\begin{array}{l}\text { Planning \& } \\
\text { Programming }\end{array}$} & Resource Allocation & 0.116 & 0.343 & 0.569 \\
\hline & $\begin{array}{c}\text { Decisions Based on } \\
\text { Condition Data } \\
\end{array}$ & 0.431 & 0.657 & 0.884 \\
\hline $\begin{array}{l}\text { Program } \\
\text { Delivery }\end{array}$ & Program Delivery & 1.000 & 1.000 & 1.000 \\
\hline
\end{tabular}


Table 4.6: Total Weights for AM Sub-Indicators

\begin{tabular}{|c|c|c|}
\hline Major Indicators & Sub-Indicators & Total Weight \\
\hline \multirow{4}{*}{$\begin{array}{l}\text { Asset Management } \\
\text { Culture }\end{array}$} & $\begin{array}{c}\text { Asset Management } \\
\text { Champions } \\
\end{array}$ & 0.053 \\
\hline & $\begin{array}{c}\text { Perception of Asset } \\
\text { Management }\end{array}$ & 0.042 \\
\hline & $\begin{array}{c}\text { Effective } \\
\text { Communication }\end{array}$ & 0.059 \\
\hline & $\begin{array}{c}\text { State Legislative } \\
\text { Mandate }\end{array}$ & 0.041 \\
\hline \multirow{4}{*}{$\begin{array}{l}\text { Quality Information } \\
\qquad \& \text { Analysis }\end{array}$} & Asset Inventory & 0.082 \\
\hline & $\begin{array}{c}\text { Common } \\
\text { Referencing System }\end{array}$ & 0.078 \\
\hline & $\begin{array}{c}\text { Up-to-Date } \\
\text { Condition Data }\end{array}$ & 0.054 \\
\hline & $\begin{array}{c}\text { Data Management } \\
\text { \& Analysis } \\
\end{array}$ & 0.050 \\
\hline \multirow{2}{*}{$\begin{array}{l}\text { Policy Goals \& } \\
\text { Objectives }\end{array}$} & $\begin{array}{c}\text { Policy Goals \& } \\
\text { Objectives }\end{array}$ & 0.171 \\
\hline & $\begin{array}{c}\text { Performance } \\
\text { Measures \& Targets }\end{array}$ & 0.129 \\
\hline \multirow{2}{*}{$\begin{array}{l}\text { Planning \& } \\
\text { Programming }\end{array}$} & Resource Allocation & 0.042 \\
\hline & $\begin{array}{c}\text { Decisions Based on } \\
\text { Condition Data } \\
\end{array}$ & 0.081 \\
\hline Program Delivery & Program Delivery & 0.118 \\
\hline
\end{tabular}




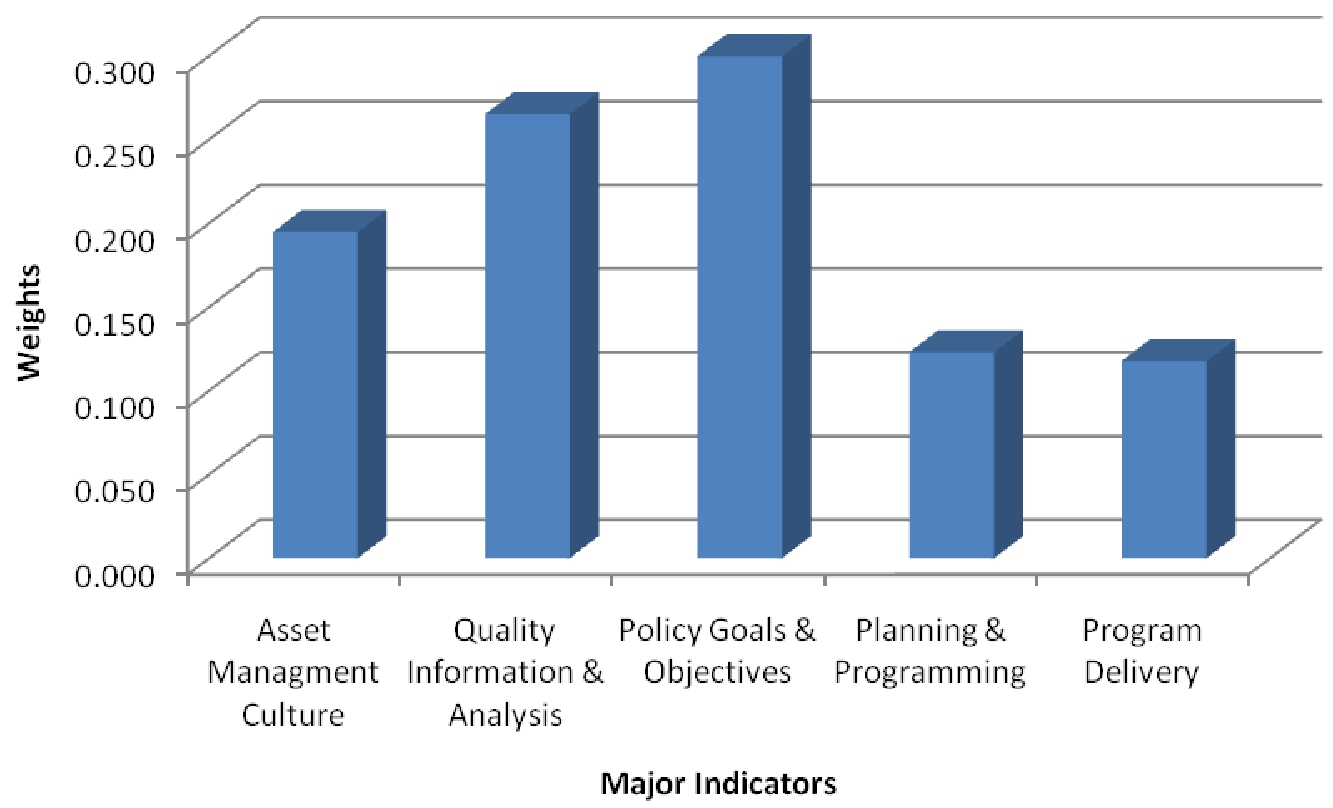

Figure 4.5: Major Indicator Weights

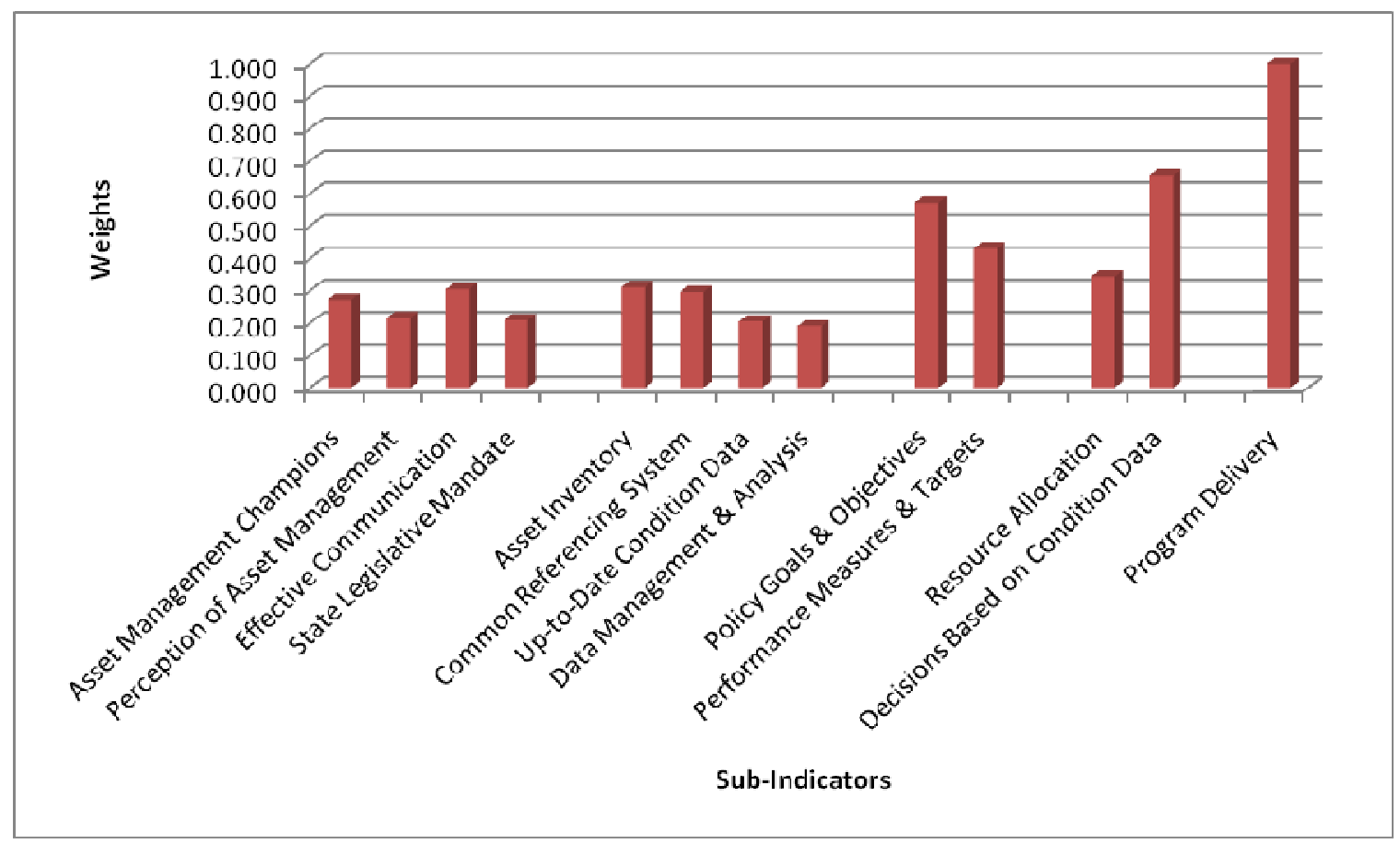

Figure 4.6: Sub-Indicator Weights 


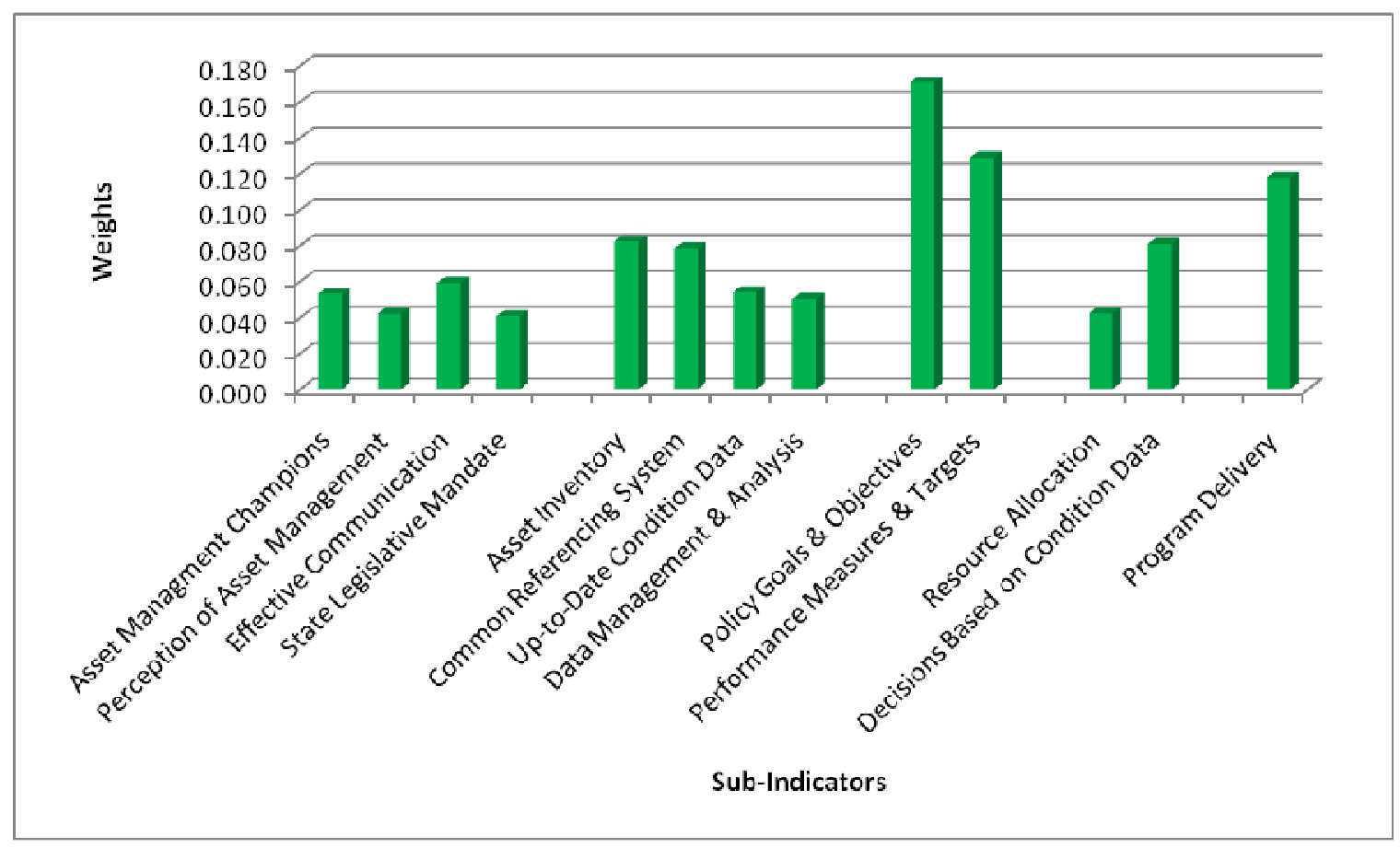

Figure 4.7: Total Sub-Indicator Weights

\subsubsection{Interpretation of Results:}

It should first be noted that the standard deviations for the major indicators and sub-indicators are very large. Figures 4.8-4.12 illustrate the scatter for the major indicators and Figures 4.13-4.24 for the sub-indicators. The standard deviation values are discussed in further detail in the Discussions section of this chapter.

It is fairly clear that Policy Goals \& Objectives is the most important major indicator. It has an average weight of 30 percent and was chosen by AM experts to be the most important indicator in 11 of 16 surveys. It is also clear that Policy Goals \& Objectives, Quality Information \& Analysis, and Asset Management Culture are the top three major indicators. In 15 of 16 surveys, the respondent selected two of these three indicators in their own top three indicators. The gap between Asset Management Culture (the third highest indicator) and Planning \& Programming (the fourth highest indicator) is 
7.2 percentage points, which is the largest gap between any two neighboring indicators in the rank order. Figure 4.5 plainly shows that these three top indicators have separated themselves from the other two. This finding is especially significant for Asset Management Culture. Each of the other major indicators has a certain amount of familiarity and name recognition because of their prominence in other AM studies. Until now, Asset Management Culture has never been formally recognized as a necessary component to an AM system. The fact that it has clearly been selected as one of the top three indicators is a significant and exciting finding for this research.

Other notable observations include Policy Goals \& Objectives and Decisions Based on Condition Data being ranked highly as sub-indicators. Besides these two, the other sub-indicator groups are rather evenly distributed. This would seem to indicate that these sub-indicators are of near equal importance.

Once the total weights for the sub-indicators were obtained, Policy Goals \& Objectives and Performance Measures \& Targets were shown to be the most important (Figure 4.7). This is reasonable when considering that their associated major indicator (Policy Goal \& Objectives) is the most highly ranked. Program Delivery is ranked as the third highest, but the total weights for the rest of the sub-indicators are within close range of each other. 


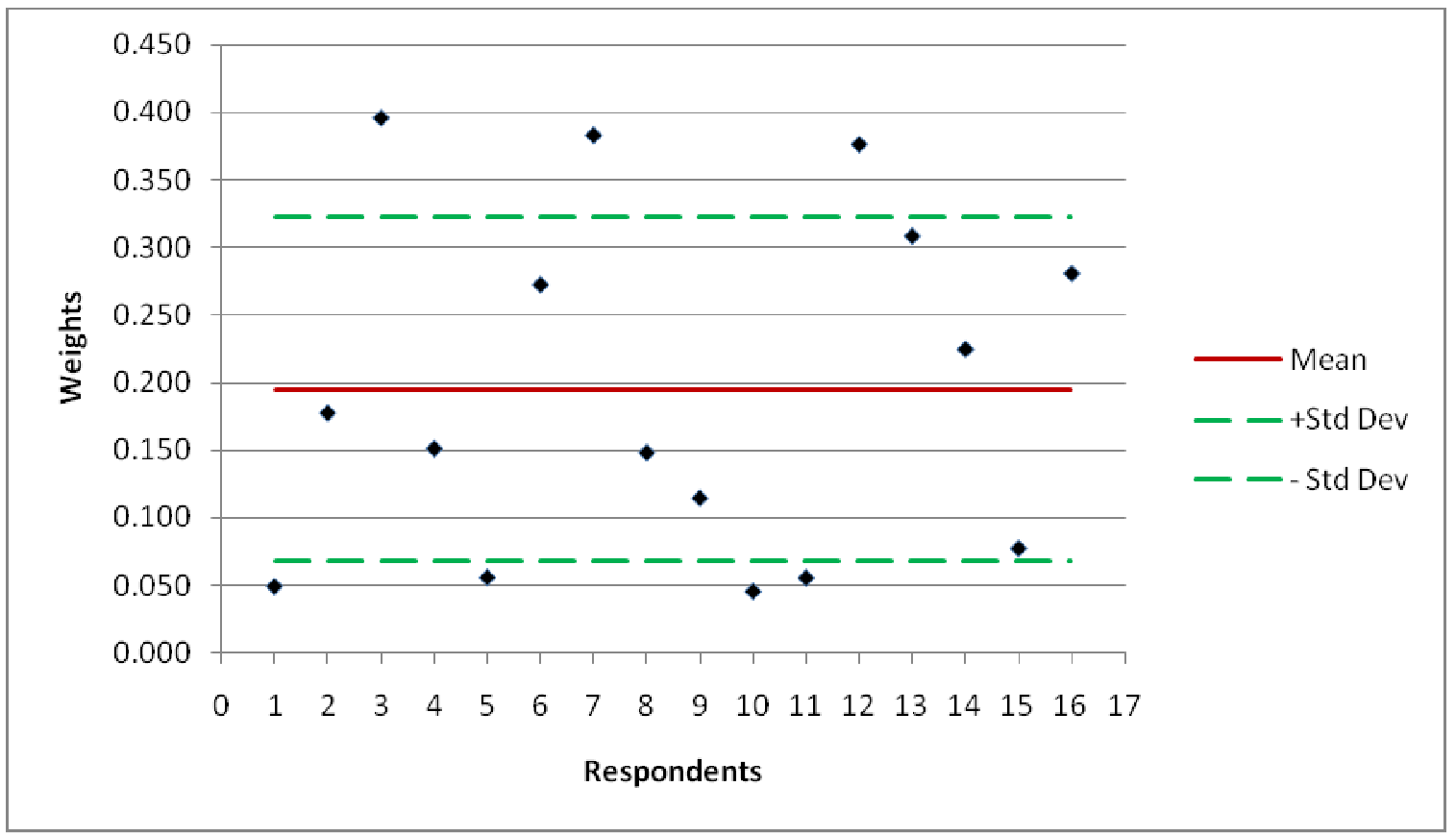

Figure 4.8: Asset Management Culture

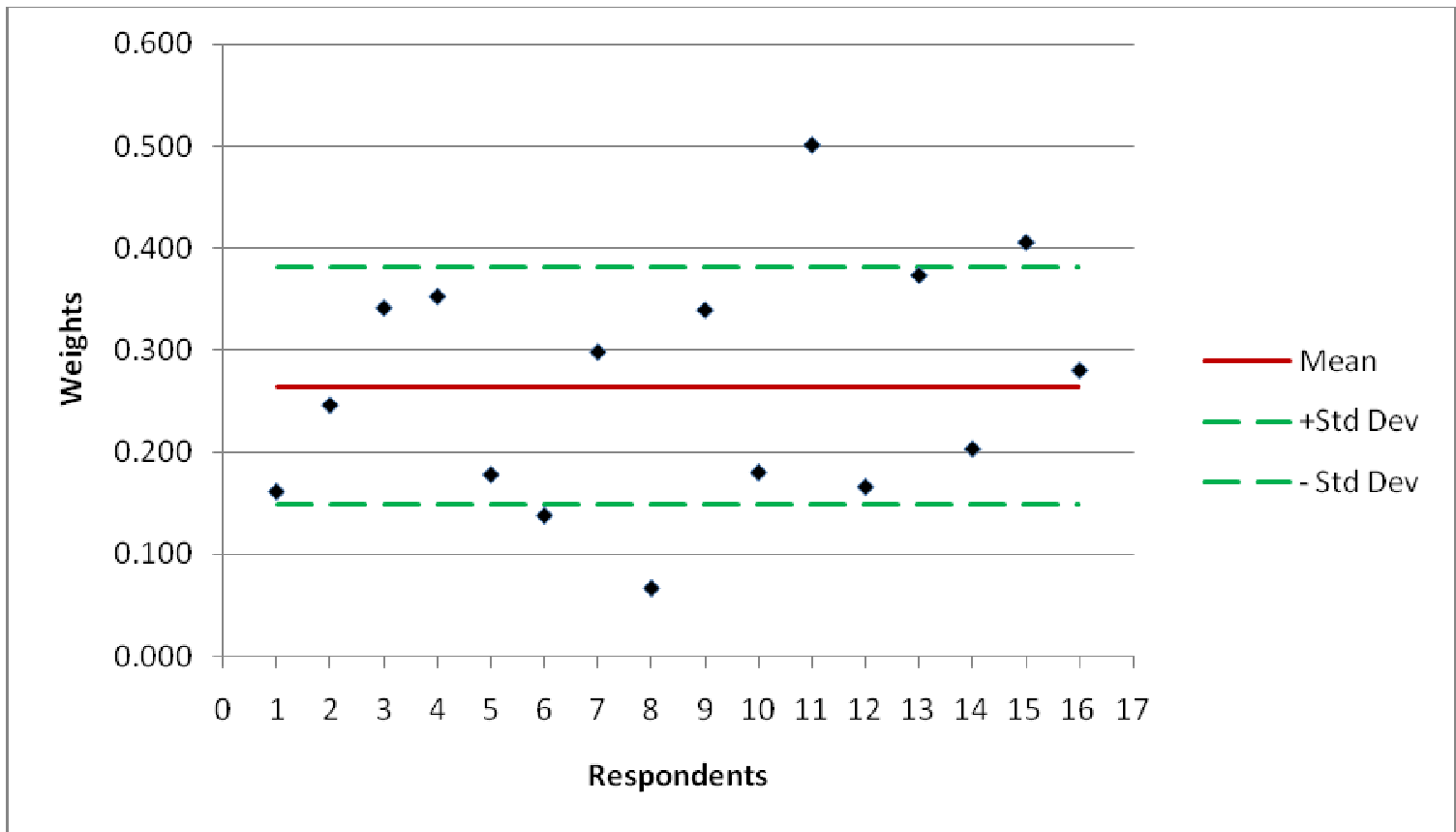

Figure 4.9: Quality Information \& Analysis 


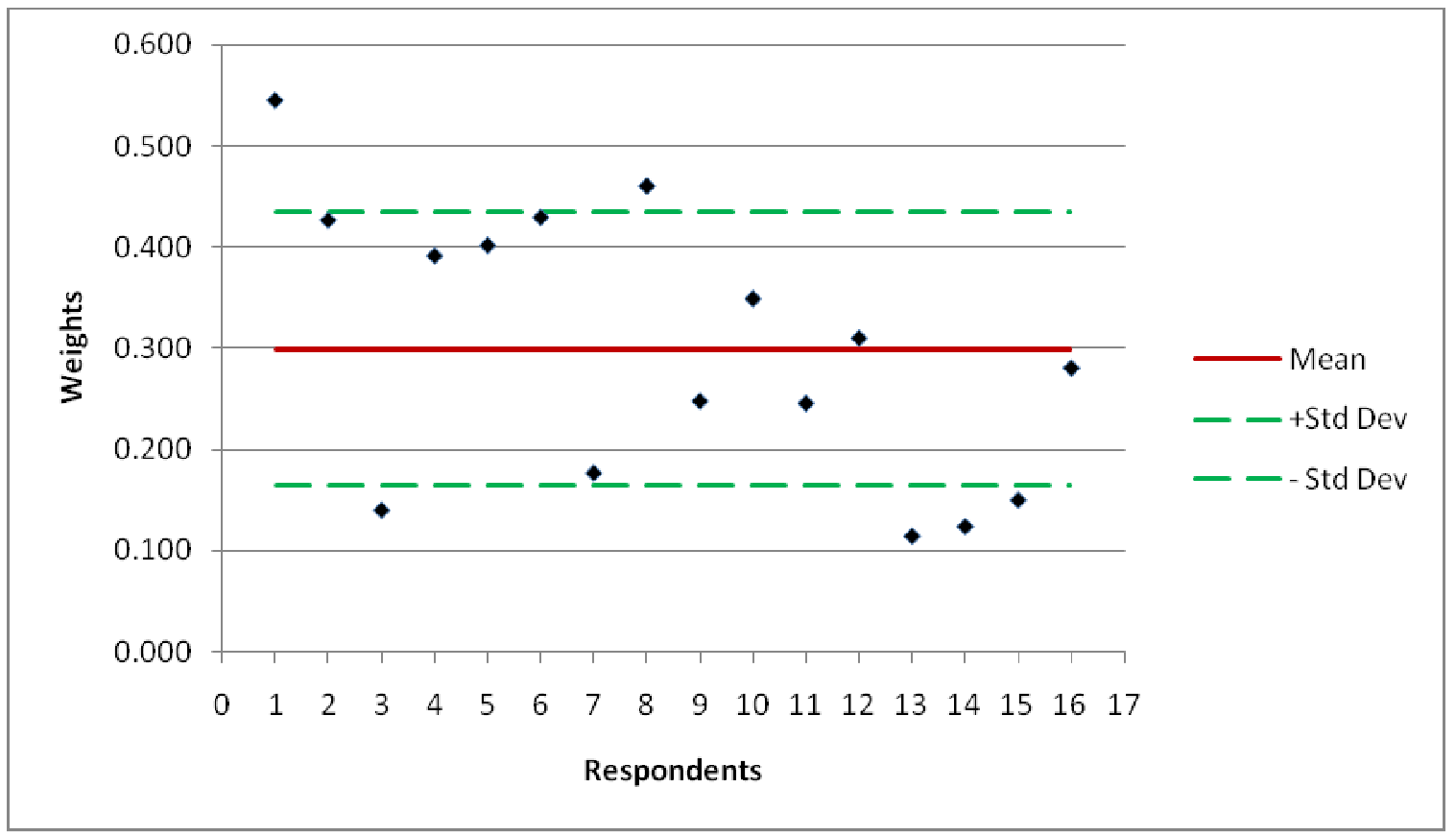

Figure 4.10: Policy Goals \& Objectives

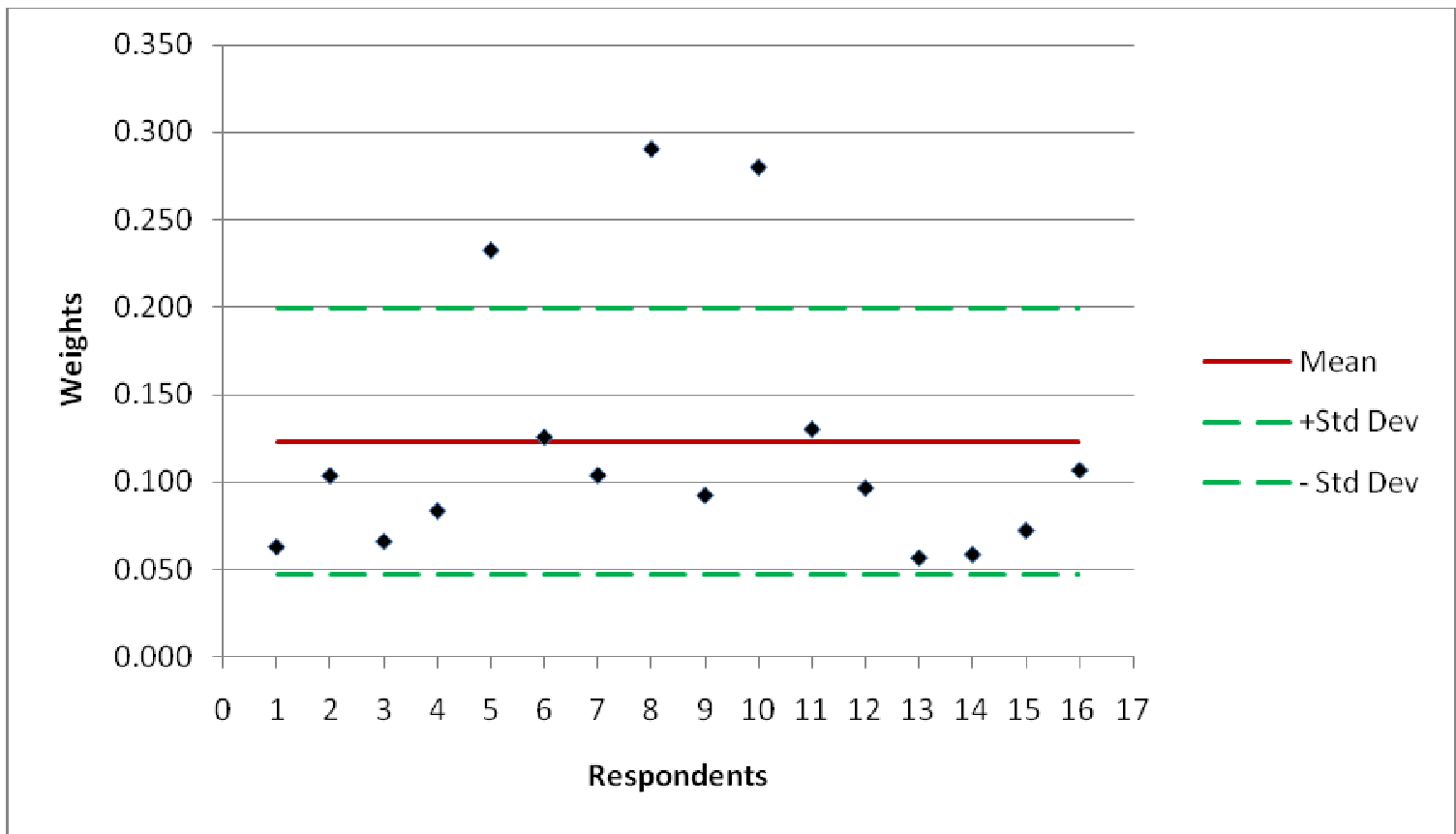

Figure 4.11: Planning \& Programming 


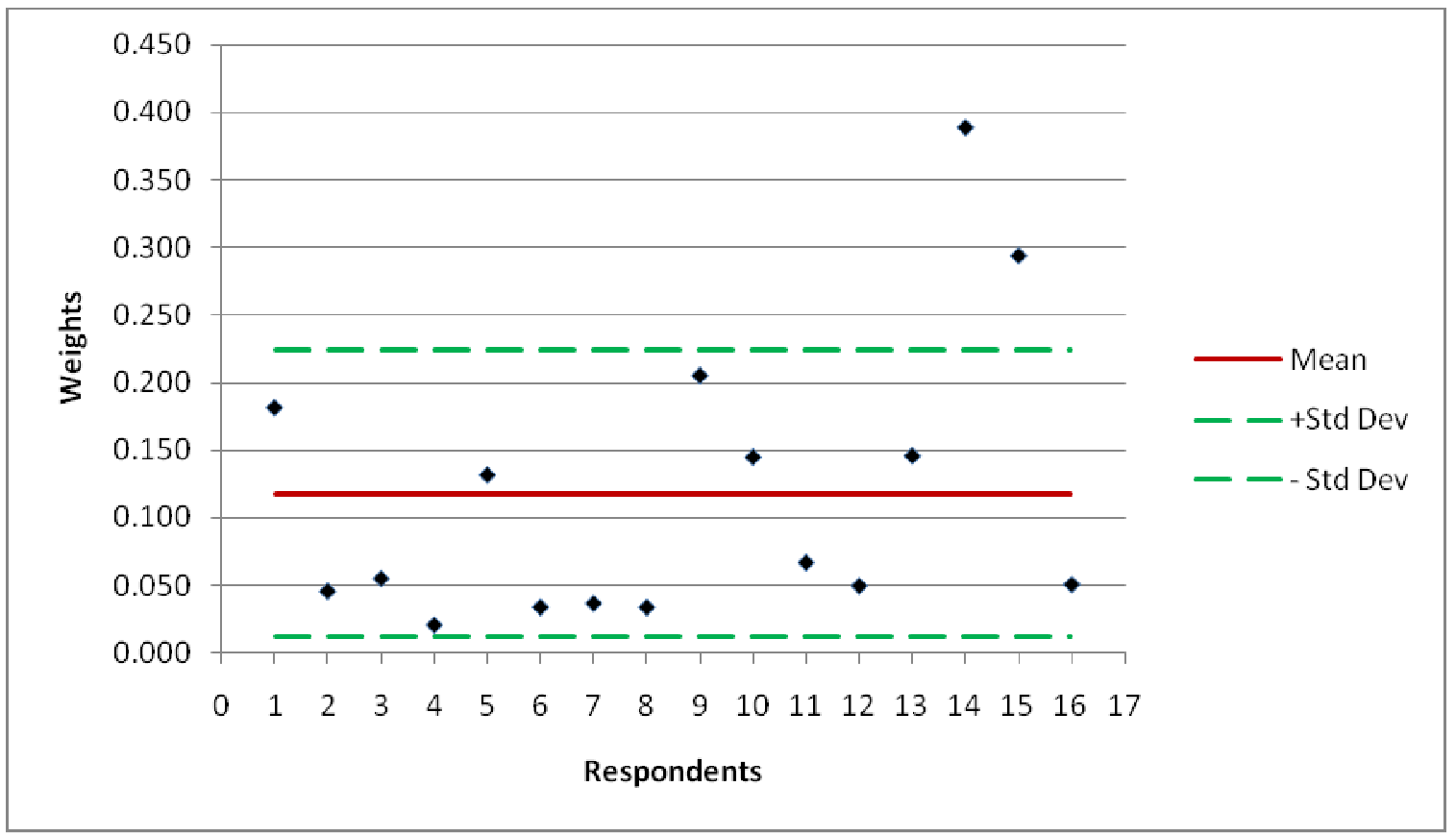

Figure 4.12: Program Delivery

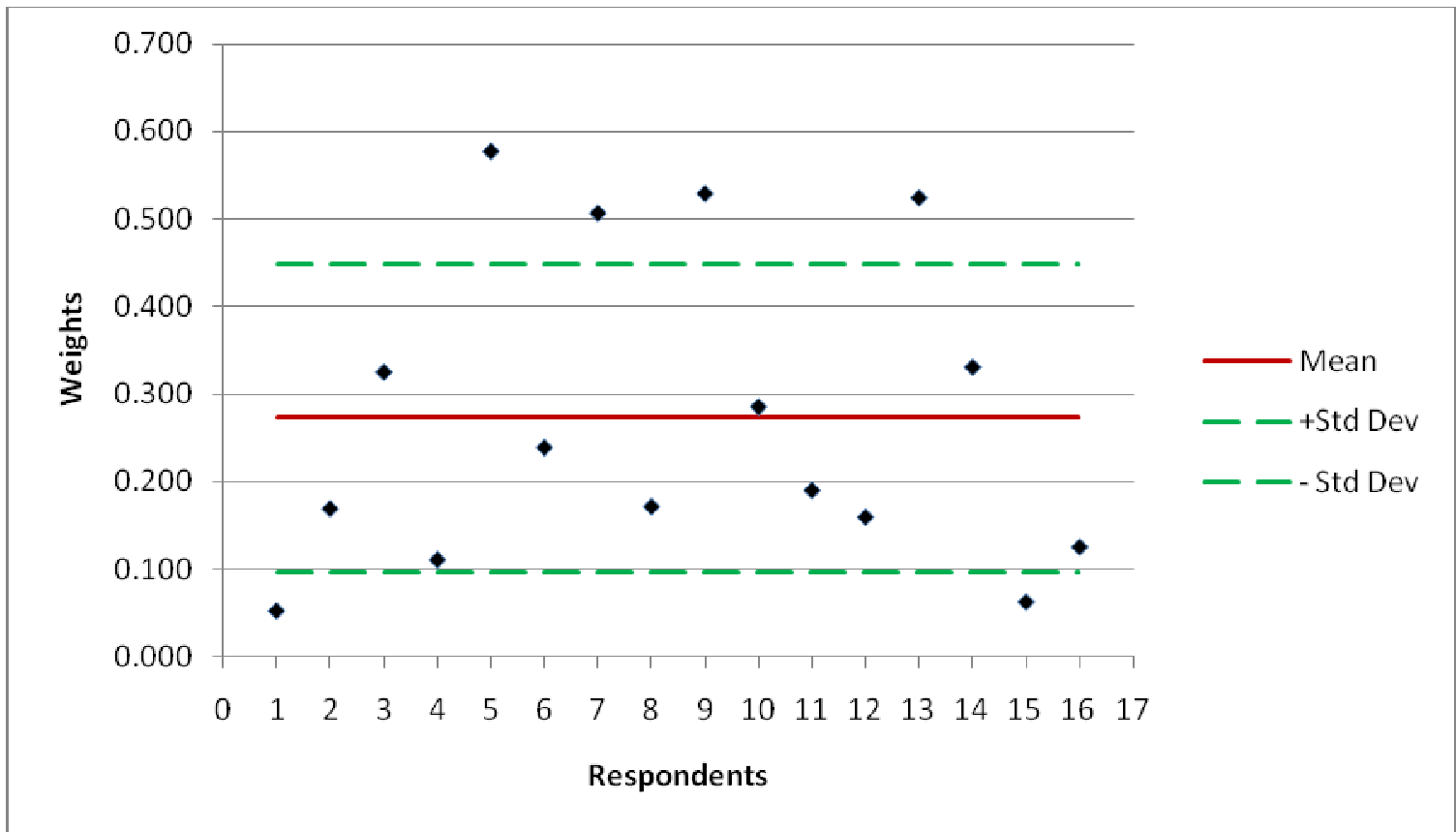

Figure 4.13: Asset Management Champions 


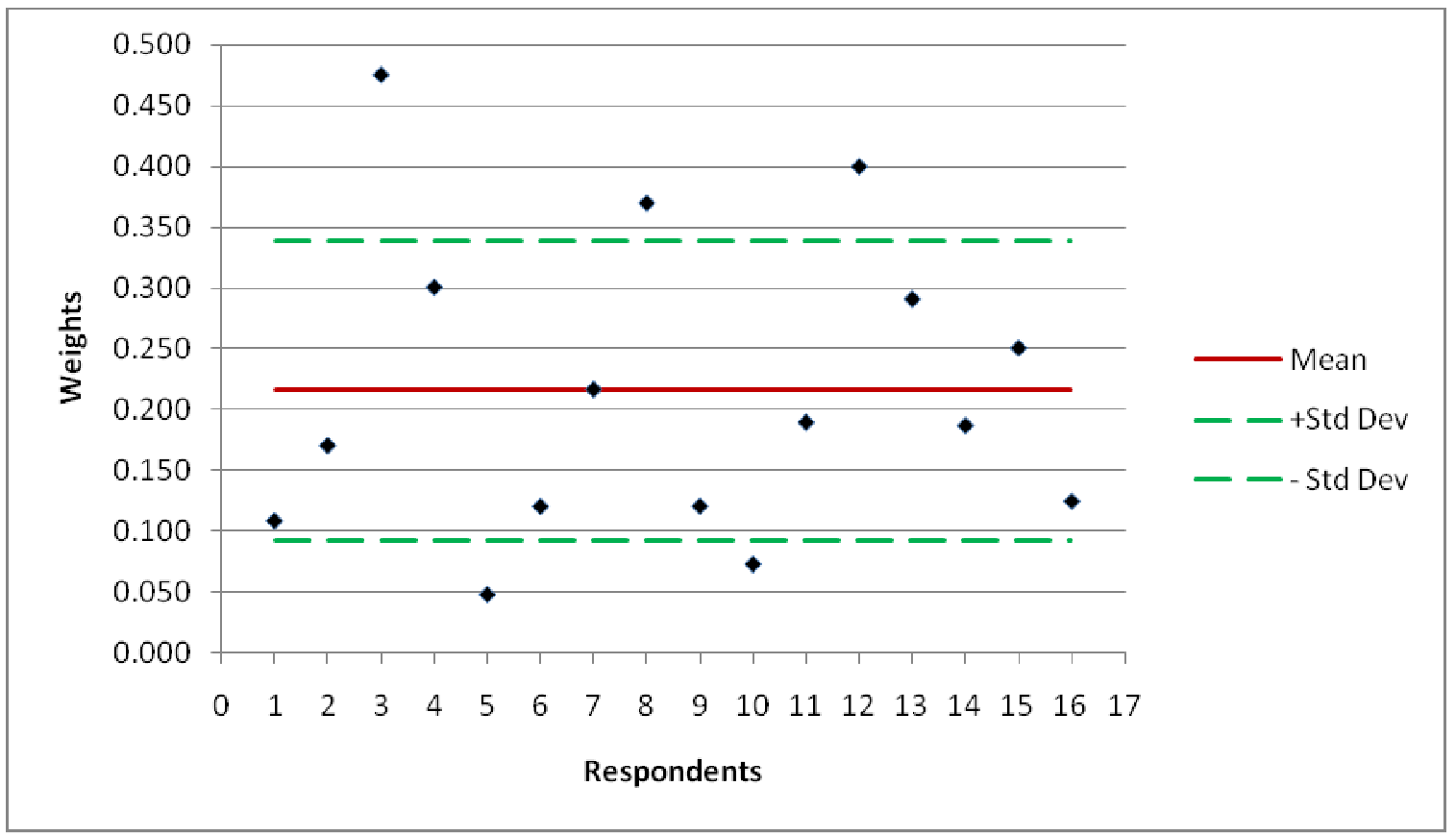

Figure 4.14: Perception of Asset Management

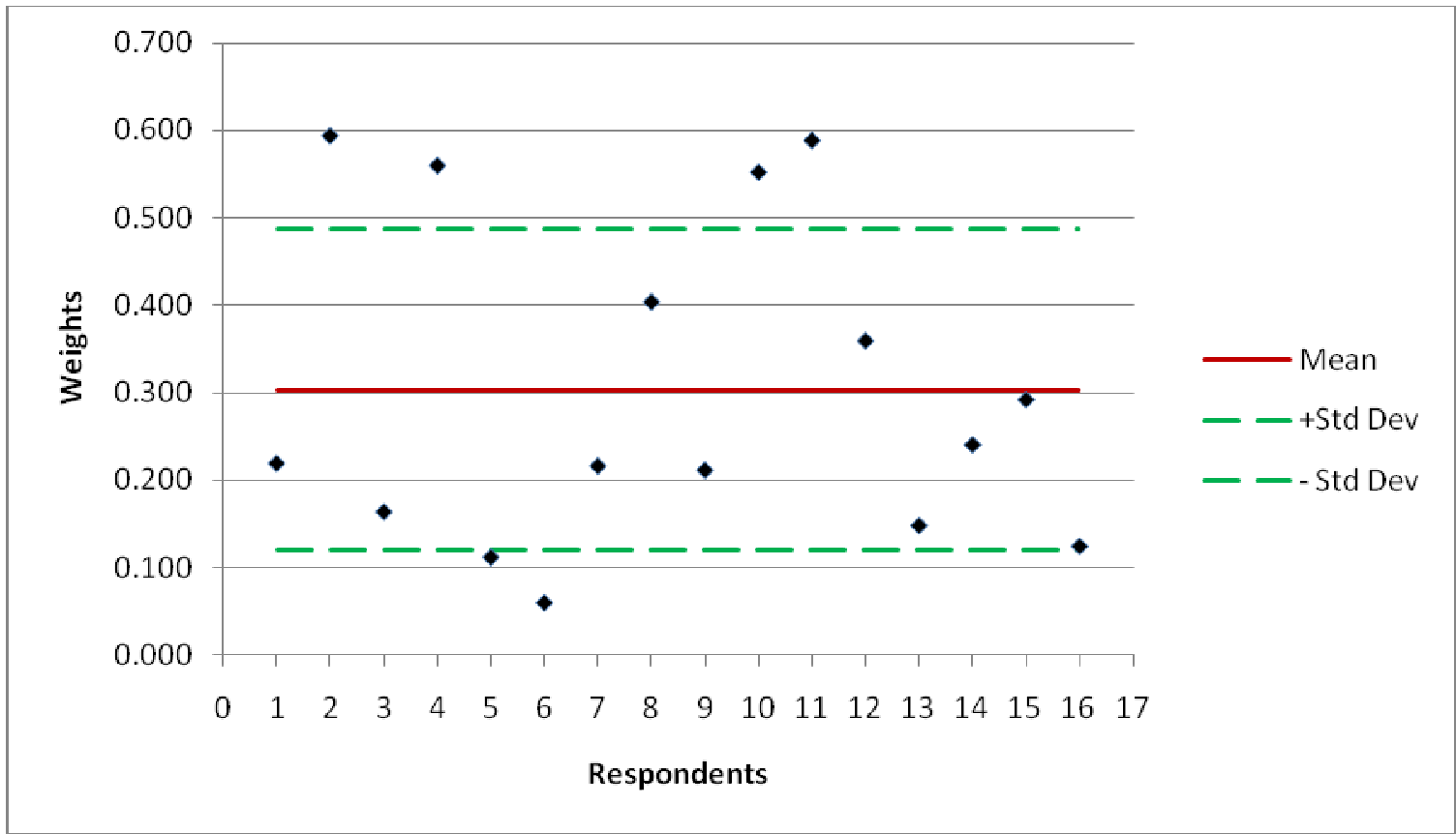

Figure 4.15: Effective Communication 


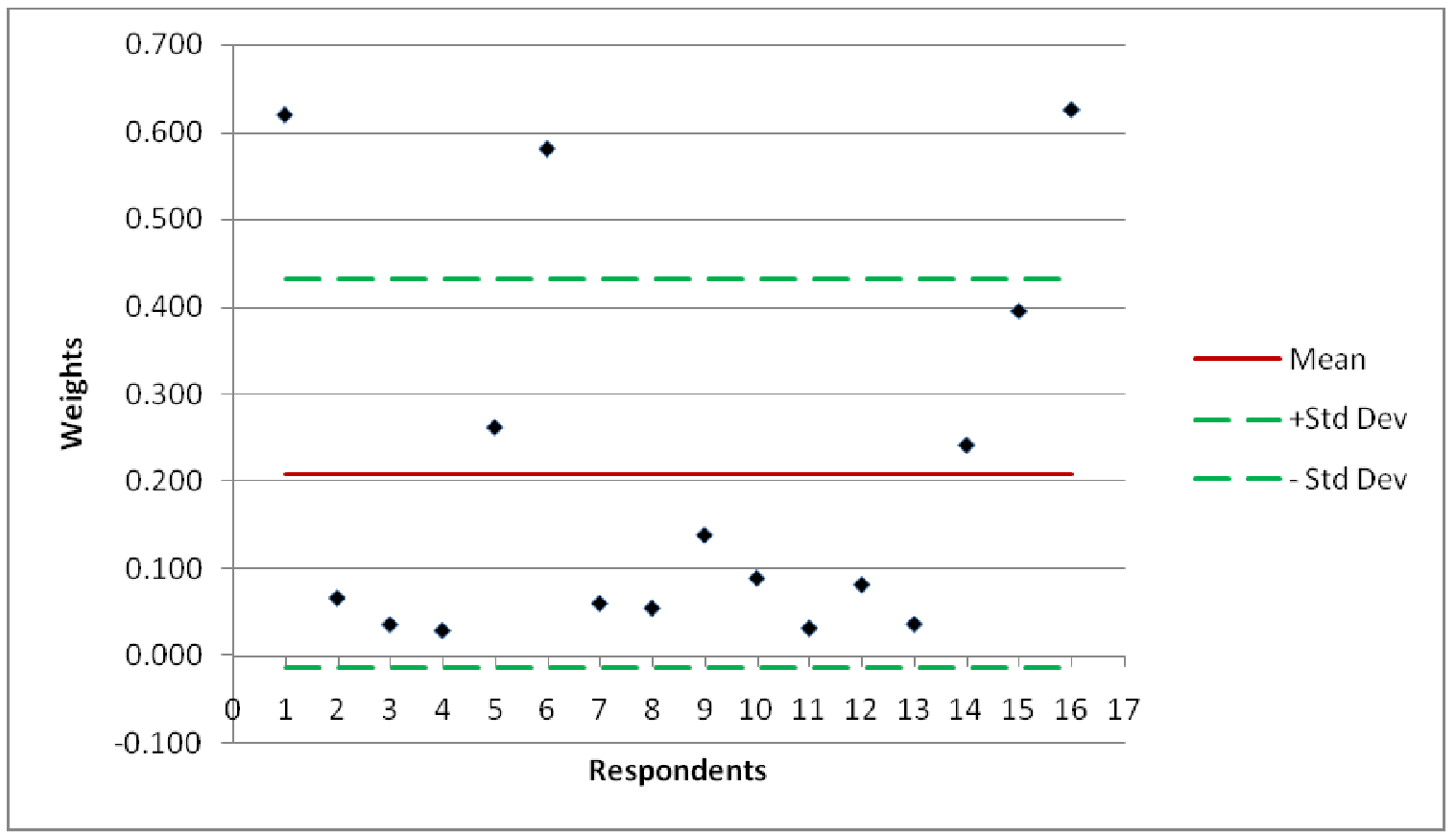

Figure 4.16: State Legislative Mandate

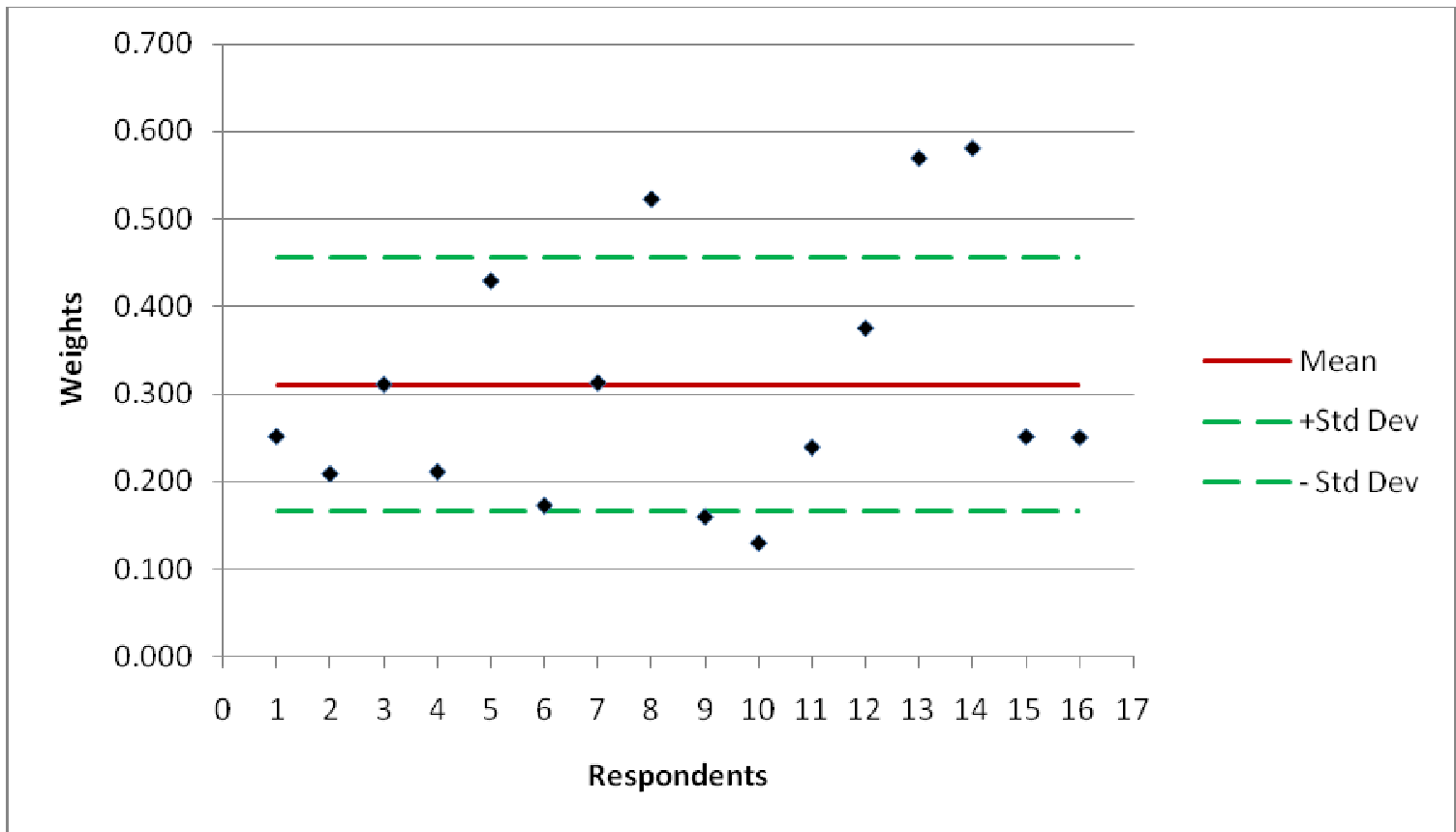

Figure 4.17: Asset Inventory 


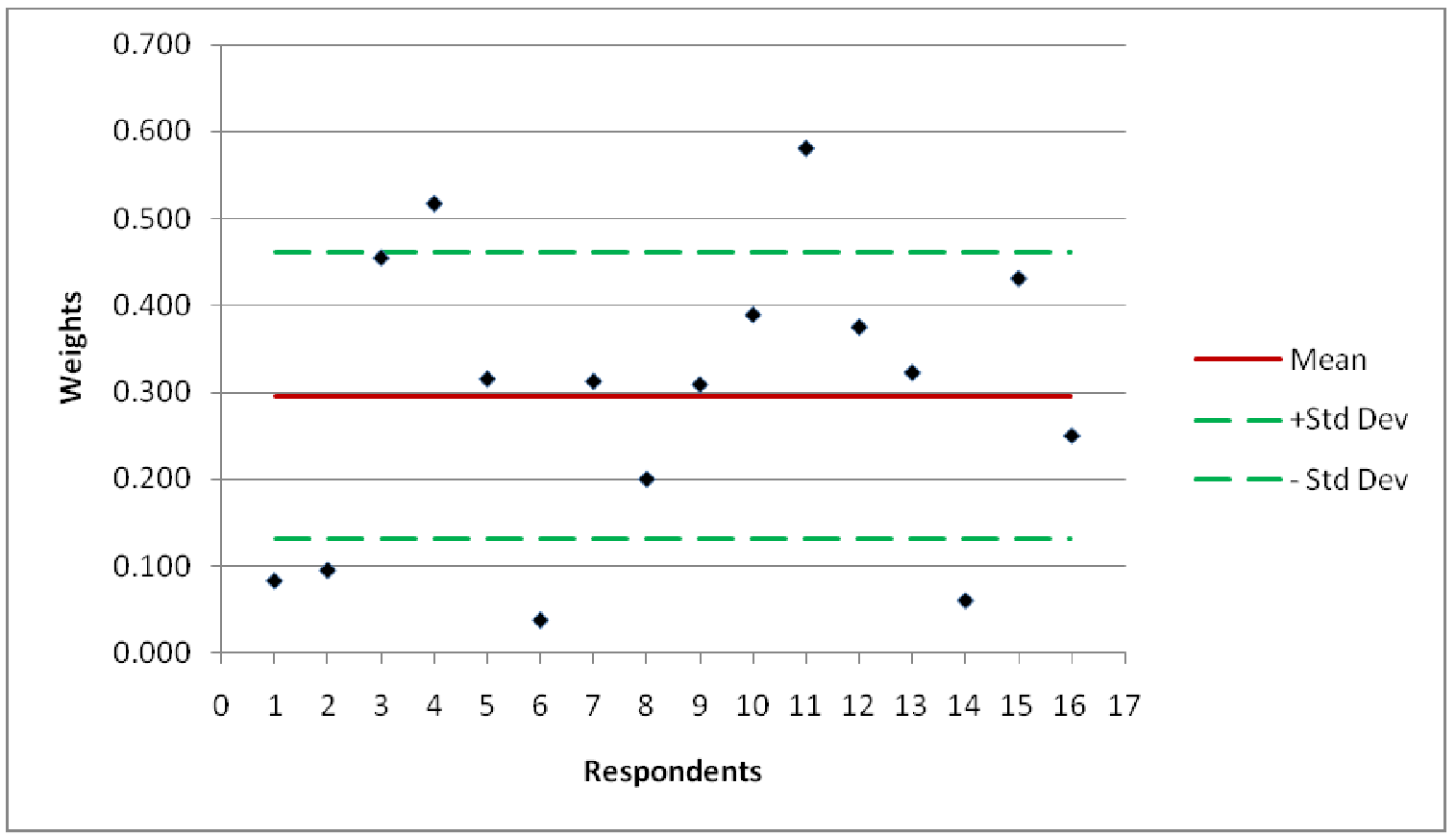

Figure 4.18: Common Referencing System

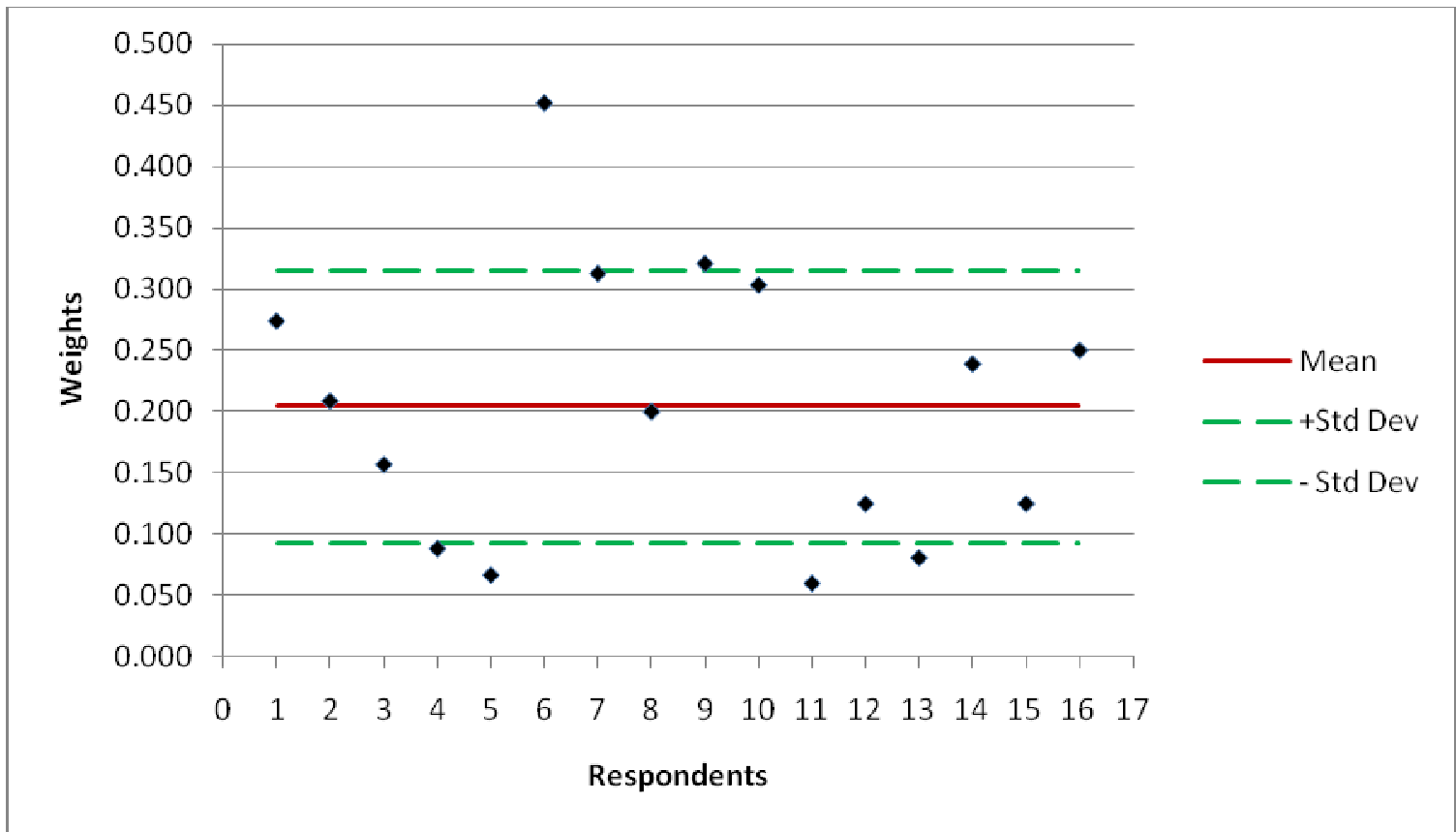

Figure 4.19: Up-to-Date Condition Data 


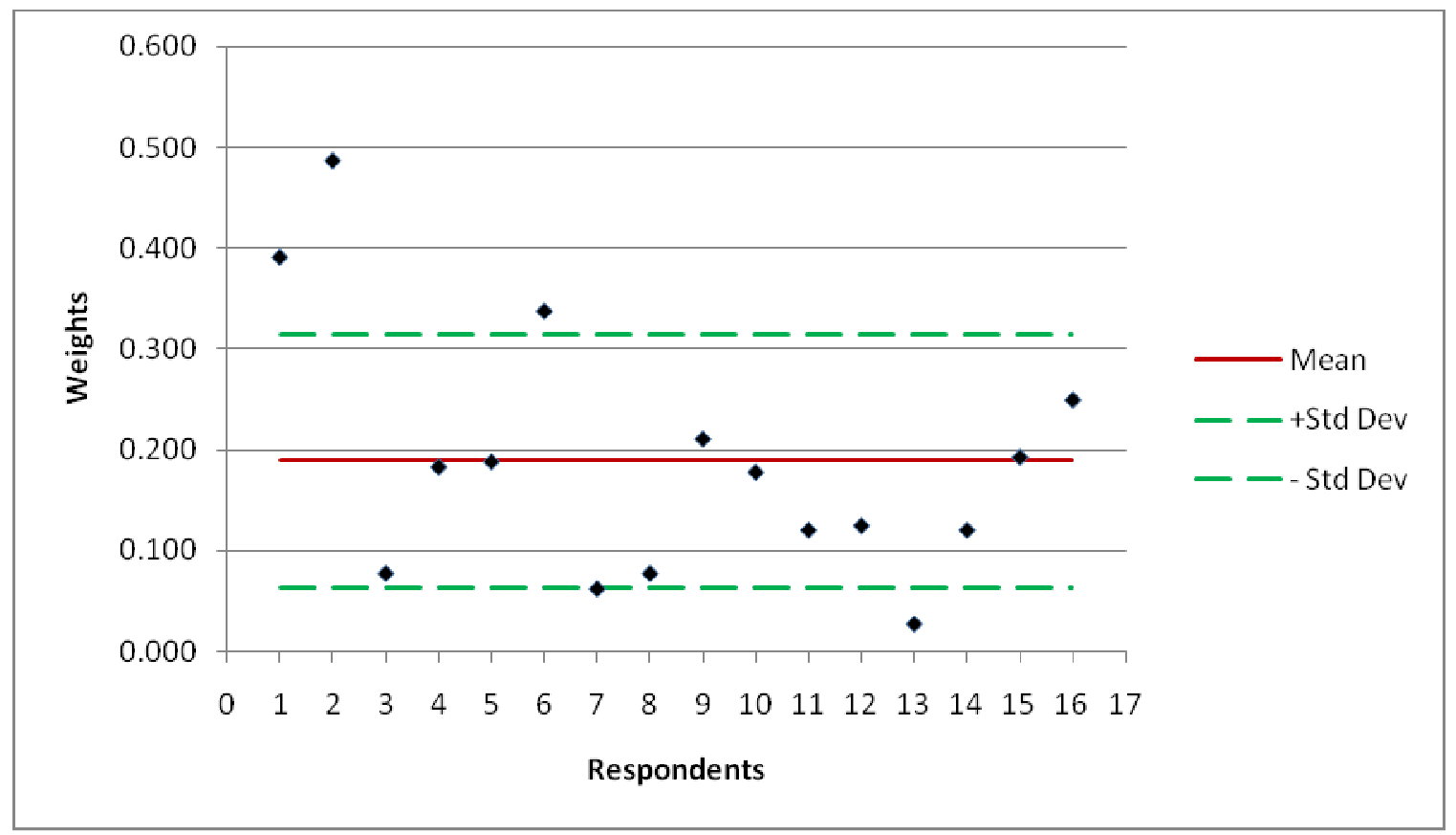

Figure 4.20: Data Management \& Analysis

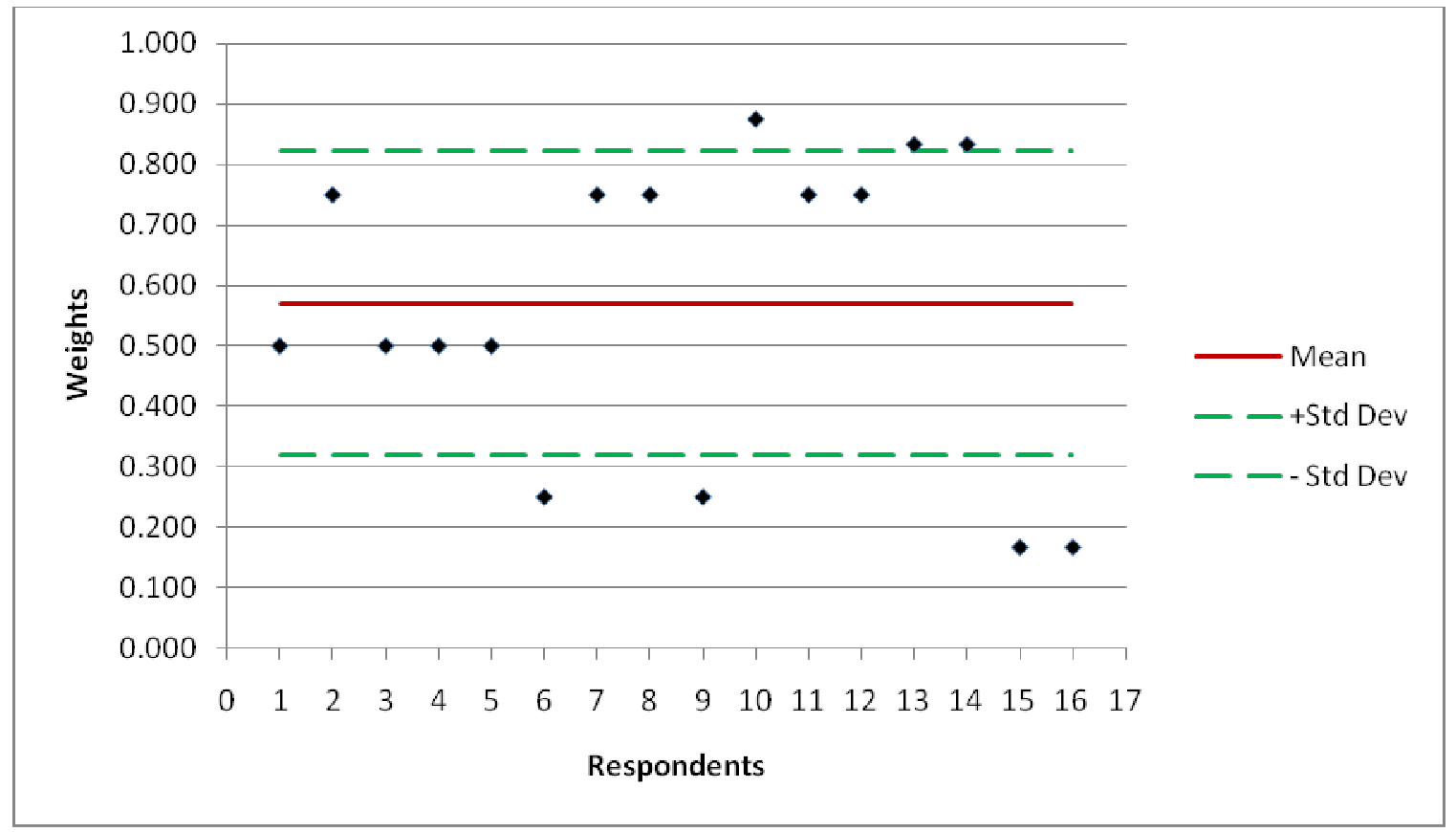

Figure 4.21: Policy Goals \& Objectives (Sub-Indicator) 


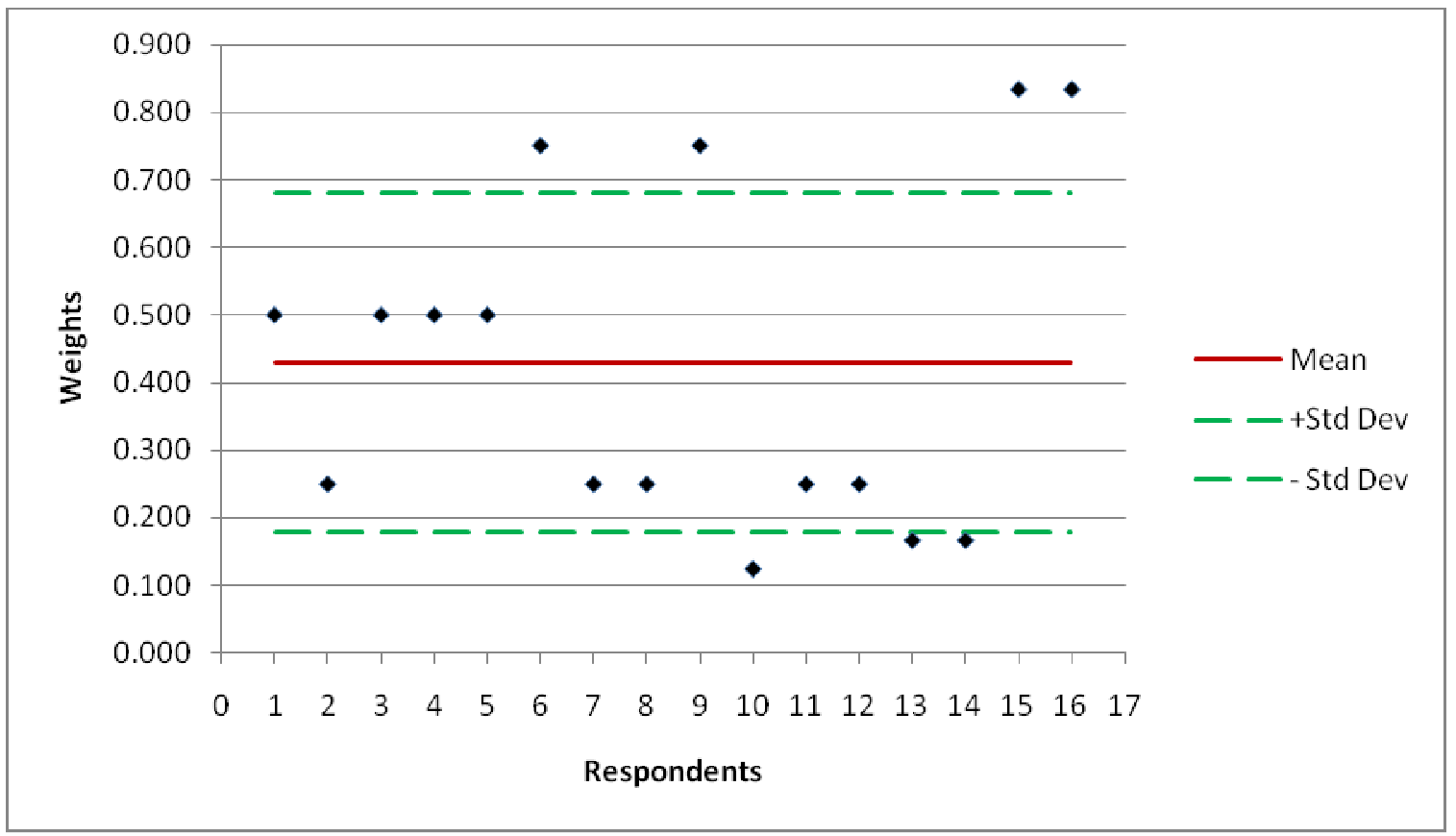

Figure 4.22: Performance Measures \& Targets

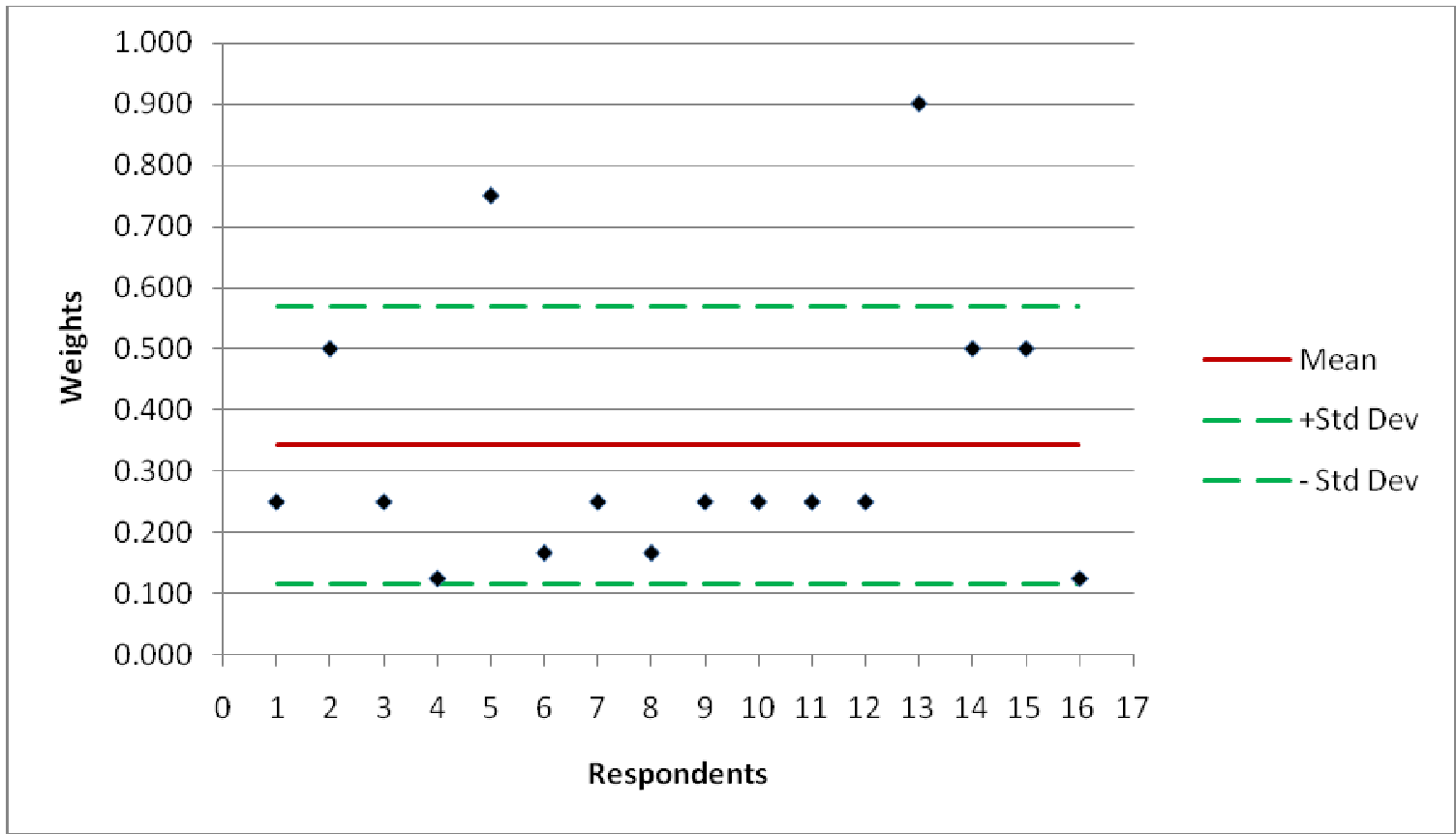

Figure 4.23: Resource Allocation 


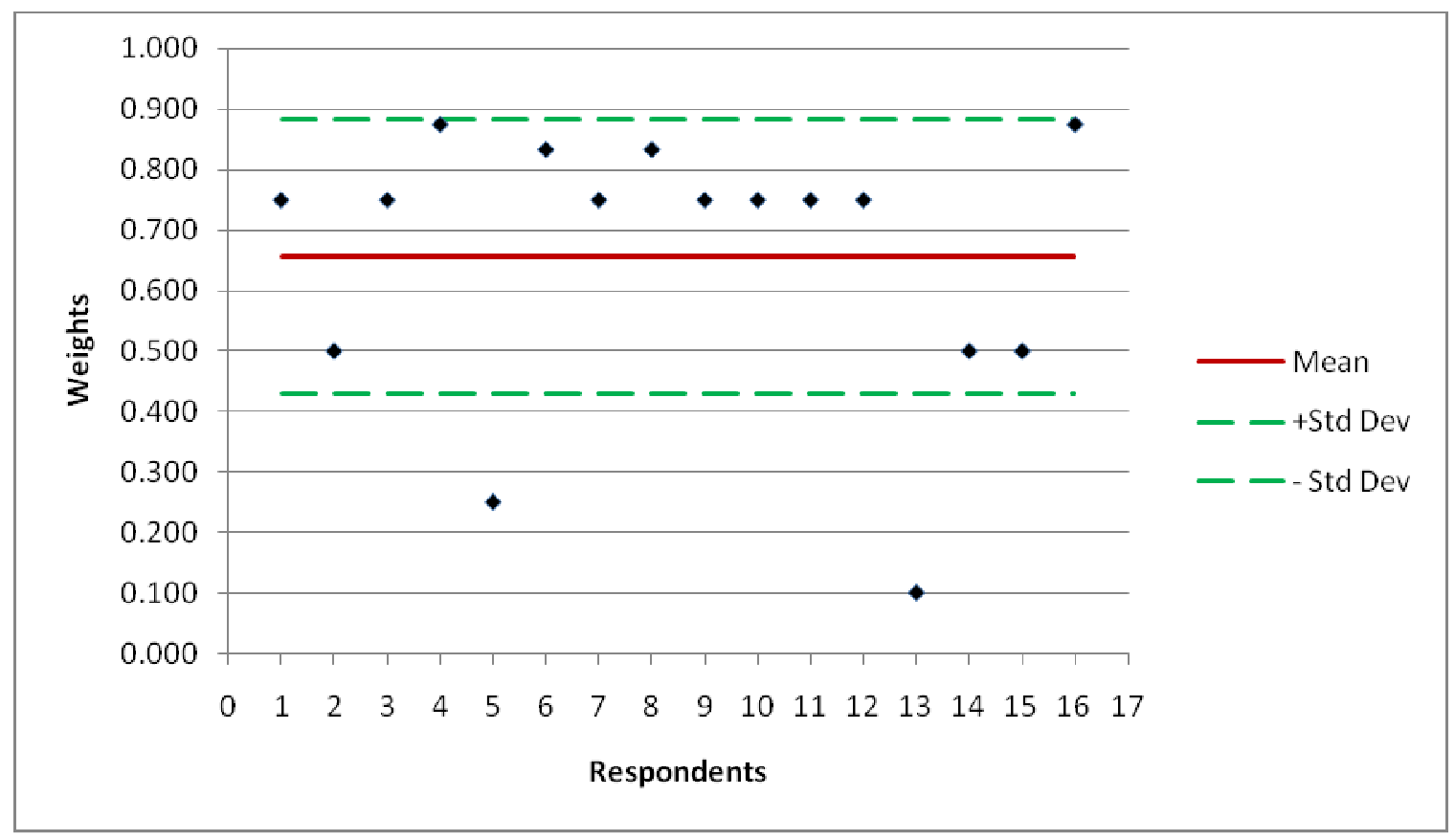

Figure 4.24: Decisions Based on Condition Data

\subsubsection{Discussions:}

It is clear that the standard deviations for the major indicators and sub-indicators are very large. These deviations indicate that the data is scattered which may demonstrate that the AM experts who participated in the survey do not agree about which indicators are the most critical when implementing an ideal AM system. However, this conclusion cannot necessarily be drawn because of imperfections in the way that AHP was applied by using a survey.

A perfect scenario for applying AHP through the use of a survey would be to conduct the survey in person with each participant. This would allow the participant to completely understand the meaning of Saaty's Fundamental Scale (Table 4.1) as well as how to accurately apply it for each comparison. However, this was not able to take place because of time and budgetary constraints. As a result, the survey sent via email and 
regular mail to allow a wide distribution and a quick response time. Even though the survey contains instructions about the Fundamental Scale and how to apply the comparisons, the research team acknowledges that mistakes in both of these areas could have easily taken place. Either AHP was not fully understood due to a lack of time spent reviewing the survey or the comparisons were applied incorrectly. In one case it was found that the comparisons had been applied backwards. For this case, it was corrected, but it would have been nearly impossible to detect this type of error for each survey.

On the surface, it appears that large standard deviations in the major indicators and sub-indicators would discredit any meaningful conclusion. However, the types of possible errors, as described earlier, could have easily affected the results of the survey and caused these deviations. Even with large standard deviations, the research team still believes that valuable conclusions can be extracted. 


\section{CHAPTER V}

\section{ASSET MANAGEMENT ASSESSMENT MODEL}

\subsection{Introduction:}

The Asset Management Assessment Model for State DOTs represents the primary deliverable of this study. The purpose of identifying the AM indicators and assigning their weights was to create an assessment tool to more accurately measure the level of AM implementation within a State DOT. This AM Assessment Model serves two purposes: (1) to be used as a diagnostic tool so that State DOTs may identify their own strengths and weaknesses within AM; and (2) to serve as an AM benchmark so that peer agencies may compare their results.

\subsection{AM Assessment Model Design:}

The AM Assessment Model used for validation can be found in Appendix B. It was determined that this model should be filled out by AM officials within a given DOT and therefore the model appears in a survey format. This survey is contained in Microsoft Excel which allows for information to be entered electronically, instant calculation of results, and ease of distribution.

The model allows for the participant to enter their name, transportation agency, years of experience, and position/title. Below this section are instructions that explain how the model should be addressed. Special attention is given to make sure the 
participant understands that the model should represent an evaluation of where their DOT currently stands with respect to AM. Many DOTs understand and are familiar with at least some AM principles and agree that those principles are important, but are not currently implementing those principles. This model intends to capture the current state of AM implementation as well as serve as an evaluation of the entire highway system, not individual management systems. This concept is imperative to communicate to the participant as they evaluate their own agency.

In the assessment model, each major indicator is listed with its associated subindicators shown beneath. For each sub-indicator, there are between one and five statements that must be evaluated. Each statement represents a benchmark level of AM implementation that was identified in conjunction with the AM indicators. The participant is to evaluate each statement on behalf of their DOT and choose whether they agree or disagree with the statement, to a varying degree (using a Likert scale). Each response has a score associated with it. They are given as: $1=$ Strongly Disagree, $2=$ Disagree, 3 = Neutral, 4 = Agree, $5=$ Strongly Agree. A "Don't Know" option is also included as one of the responses. This response receives a score of " 0 " meaning that no knowledge about the subject is the least valuable in this assessment. A response of "Strongly Agree" will yield a score of "5," which is the highest possible score. The same scoring system was used by Ruikar (2005) as a way to measure a construction company's e-readiness. His model was also implemented in a questionnaire-survey format.

For each set of statements that fall beneath a given sub-indicator, an average score is calculated. This average score is multiplied by the given each sub-indicator's weight to obtain a sub-score. This sub-score is then divided by the maximum possible score for that 
given sub-indicator which yields a percentage or grade for each. To obtain the grade for each major indicator, the sub-scores for each sub-indictor are totaled and divided by the maximum amount of points available within that major indicator. In a similar fashion, an overall grade is calculated. A 100 percent in any of the sub-indictors or major indicators would signify a perfect score in that area of AM. This means an agency is at the benchmark level of AM implementation for that given indicator.

In the Excel file that contains the assessment model, there are four additional worksheets which display the results. The first worksheet is entitled 'Results' which shows the overall grade, major indicator grades, and sub-indicator grades as percentages. A graph representing the grades of each of the major indicators is also shown. The next worksheet, entitled 'Score Breakdown' offers a more detailed view of the grades. On this sheet, the response for each statement can be seen as well as the average score and grade for each sub-indicator. The next two worksheets deal with gap analysis, which is discussed in Section 5.4.

\subsection{Validation:}

Once the AM Assessment Model was developed, it was tested on five DOTs in order to validate the model's effectiveness. The model would be deemed effective if it returned grades that were in line with what could be expected from DOTs at known AM implementation levels. While it is currently not possible to know the exact level of AM implementation at a given DOT, it is known that certain DOTs are leaders in this area, others are in the initial stages, and some have not started any AM implementation. 
Five DOTs were chosen to test and validate the assessment model. Three broad AM implementation levels were chosen (high, medium, low) and DOTs from each of these three levels were selected. The "high" designation represents DOTs that are advanced in AM and have been practicing AM principles for a number of years. The "medium" level represents DOTs that are familiar with AM practices and are implementing some of these practices, but still have room for growth and improvement. They understand what needs to be done, but do not currently have the capabilities to perform all of these tasks. The "low" level represents DOTs that have a limited understanding of AM principles and are currently doing very little or nothing to implement these practices. The five DOTs that were chosen are shown as anonymous, but are represented by the given names and predetermined implementation levels in Table 5.1. The purpose of this validation procedure was to determine if the AM Assessment Model would return grades for each of these DOTs that were within the predetermined range. It would be expected that DOTs \#1 and \#2 would have higher grades than DOTs \#3 and \#4, all of which should be higher than DOT \#5.

Table 5.1: State DOTs Used for Validation

\begin{tabular}{|c|c|}
\hline $\begin{array}{c}\text { Predetermined AM Implementation } \\
\text { Level }\end{array}$ & State DOT \\
\hline \hline \multirow{3}{*}{ High } & DOT \#1 \\
\cline { 2 - 3 } & DOT \#2 \\
\hline \hline Medium & DOT \#3 \\
\hline \hline Low & DOT \#4 \\
\hline
\end{tabular}


Each of the five DOTs that were selected for this validation test was first contacted and an AM representative within the agency was identified. That person was informed about the study and then emailed a copy of the AM Assessment Model. The copy of the model that was sent to the participants did not include any of the results or gap analysis worksheets. This was an attempt to make the model as objective as possible. Without these worksheets, the participants could not check their score and then revise their answers in order to obtain a higher score. However, once the model was completed, the participants had the choice to request a copy of their results.

\subsubsection{Overall Analysis of the Validation Test:}

The results of the validation test matched the predetermined AM implementation level for each DOT. While this predetermined AM implementation level was only a subjective value based on prior knowledge about the given agency's AM practices, it at least provided a starting point from which to compare the results from the AM Assessment Model. The purpose of the model is to quantify the level of AM implementation within a State DOT. The results from the validation test, shown in Table 5.2, reveal the AM assessment grades for each DOT. These grades match the predetermined AM implementation level for each agency. DOTs \#1 and \#2 are clearly the two highest scores, followed by DOTs \#3 and \#4, with DOT \#5 having the lowest grade. This is also represented in Figure 5.1. Based on the overall AM assessment grades, the DOTs scored within their expected ranges. However, a more detailed analysis of the major indicators and sub-indicators is necessary to discover how the top DOTs separated themselves from the others. 
Table 5.2: Overall Validation Results

\begin{tabular}{|c||c||c|}
\hline \multicolumn{1}{|c||}{ Predetermined AM } & State DOT & AM Assessment Grade \\
\hline \hline \multirow{2}{*}{ Implementation Level } & & $85.6 \%$ \\
\cline { 2 - 3 } & DOT \#1 & $78.1 \%$ \\
\hline \multirow{2}{*}{ Medium } & DOT \#2 & $65.6 \%$ \\
\cline { 2 - 3 } & DOT \#3 & $61.7 \%$ \\
\hline \hline Low & DOT \#4 & $51.4 \%$ \\
\hline
\end{tabular}

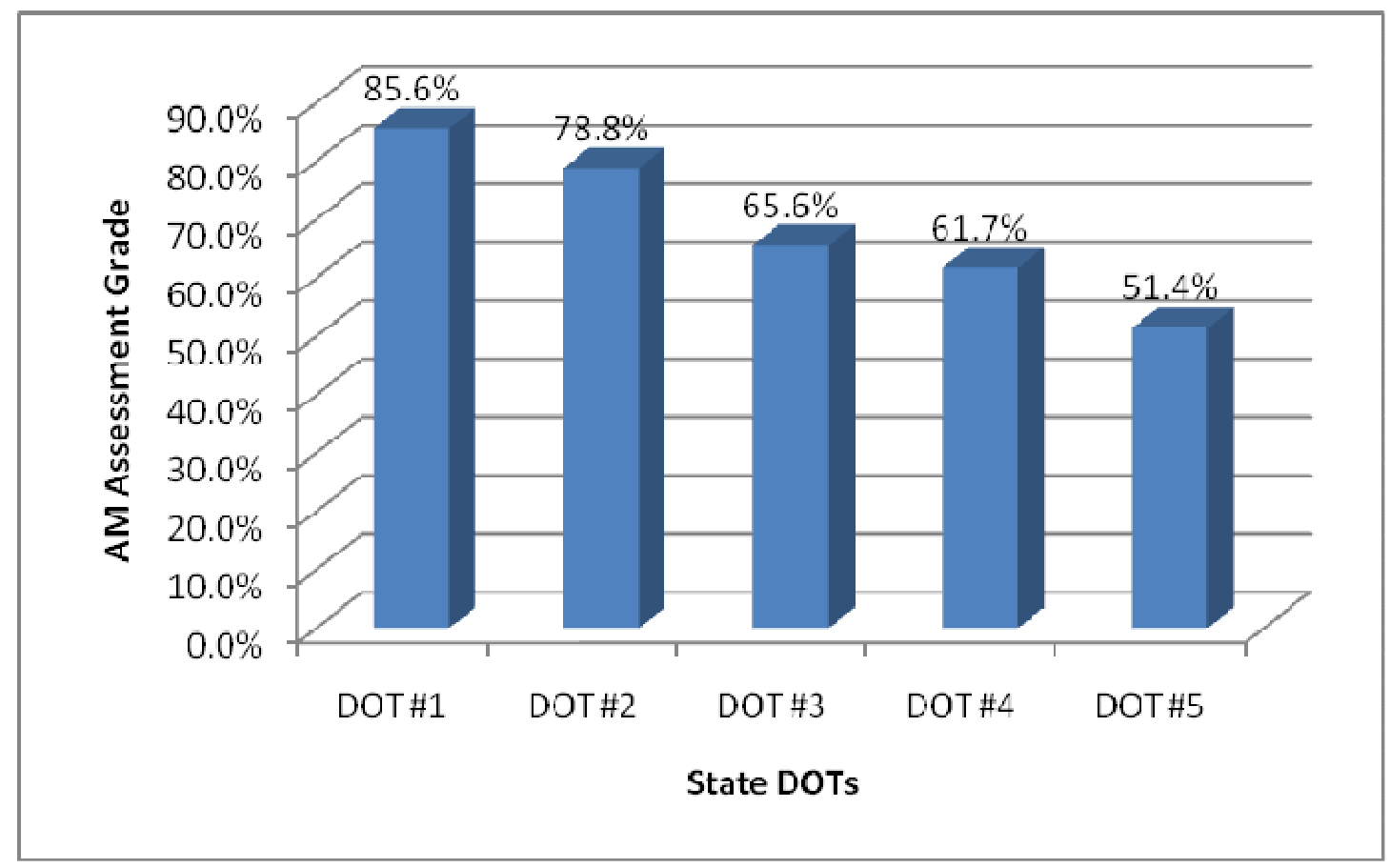

Figure 5.1: Overall Validation Results

\subsubsection{Major Indicator Analysis:}

It is evident that the top two DOTs (DOTs \#1 and \#2) have separated themselves from the other three. Table 5.3 shows the major indicator scores for each of the DOTs 
and Figures 5.2-5.6 graphically represents these results. The top two DOTs scored at least 70 percent in a combined 9 out of 10 major indicators. Of those 9, grades for 6 major indicators were 80 percent or higher. Conversely, the three remaining DOTs scored above 70 percent in only 2 of 15 combined major indicators among those agencies. Also, among the two most heavily-weighted indicators (Policy Goals \& Objectives, Quality Information \& Analysis) the top two DOTs scored at least 74.9 percent or higher.

When examining the AM Culture grades, it is evident that DOT \#1 has a lower grade compared to its other major indicators. This is because DOT \#1 answered "Don't Know" on both statements concerning AM Champions, thereby lowering their AM Culture grade. The AM representative at DOT \#1 informed the research team that the top leadership had recently changed and therefore he was not certain about the new leadership's perspective on AM. This was an honest assessment of the department at the time of the validation test. However, had the validation test been given several months later, it is likely that the AM Culture grade for DOT \#1 would be much higher.

Table 5.3: Major Indicator Grades

\begin{tabular}{|c|c||c|c|c||c||}
\hline & DOT \#1 & DOT \#2 & DOT \#3 & DOT \#4 & DOT \#5 \\
\hline \hline Asset Management Culture & $64.3 \%$ & $85.4 \%$ & $65.8 \%$ & $59.4 \%$ & $52.2 \%$ \\
\hline \hline Quality Information \& Analysis & $93.3 \%$ & $74.9 \%$ & $70.5 \%$ & $61.3 \%$ & $57.6 \%$ \\
\hline \hline Policy Goals \& Objectives & $90.3 \%$ & $78.3 \%$ & $70.9 \%$ & $66.2 \%$ & $46.3 \%$ \\
\hline \hline Planning \& Programming & $96.6 \%$ & $76.6 \%$ & $59.8 \%$ & $56.6 \%$ & $60.2 \%$ \\
\hline \hline Program Delivery & $80.0 \%$ & $80.0 \%$ & $46.7 \%$ & $60.0 \%$ & $40.0 \%$ \\
\hline
\end{tabular}




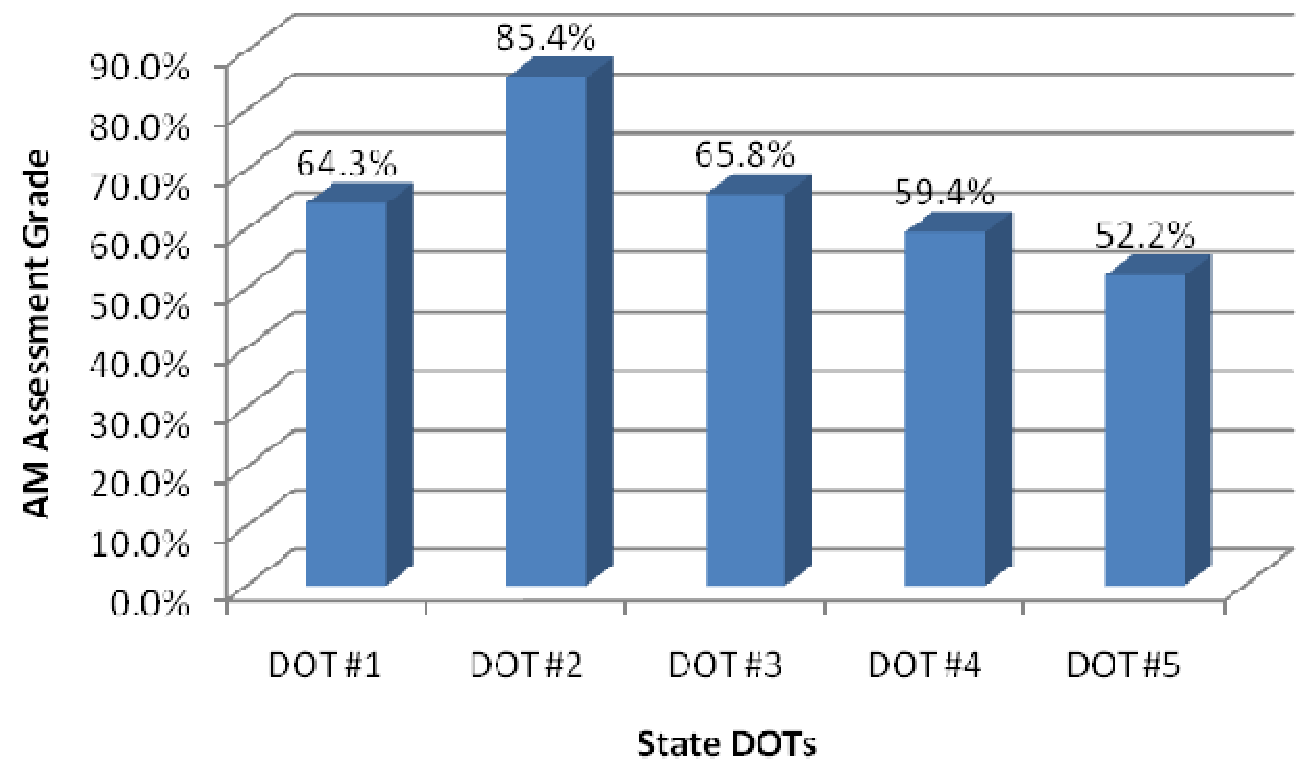

Figure 5.2: Asset Management Culture Grades

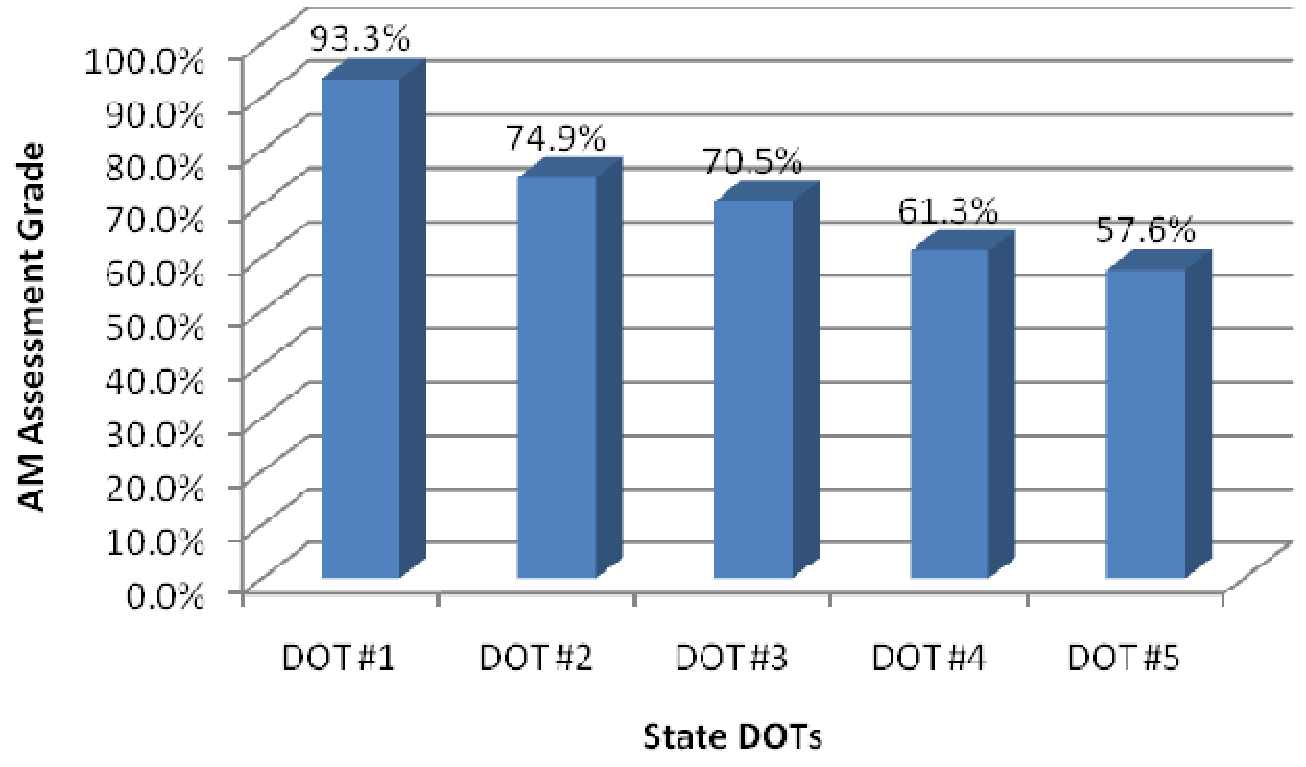

Figure 5.3: Quality Information \& Analysis Grades 


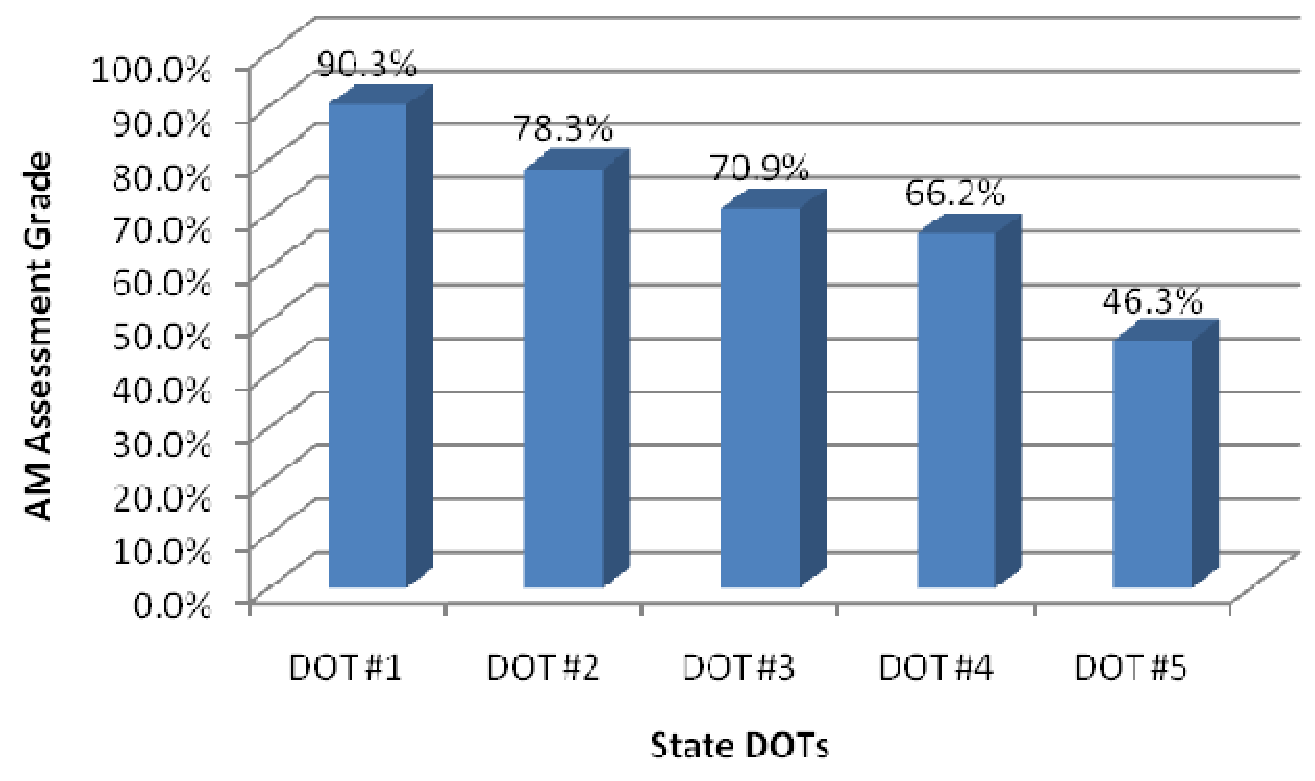

Figure 5.4: Policy Goals \& Objectives Grades

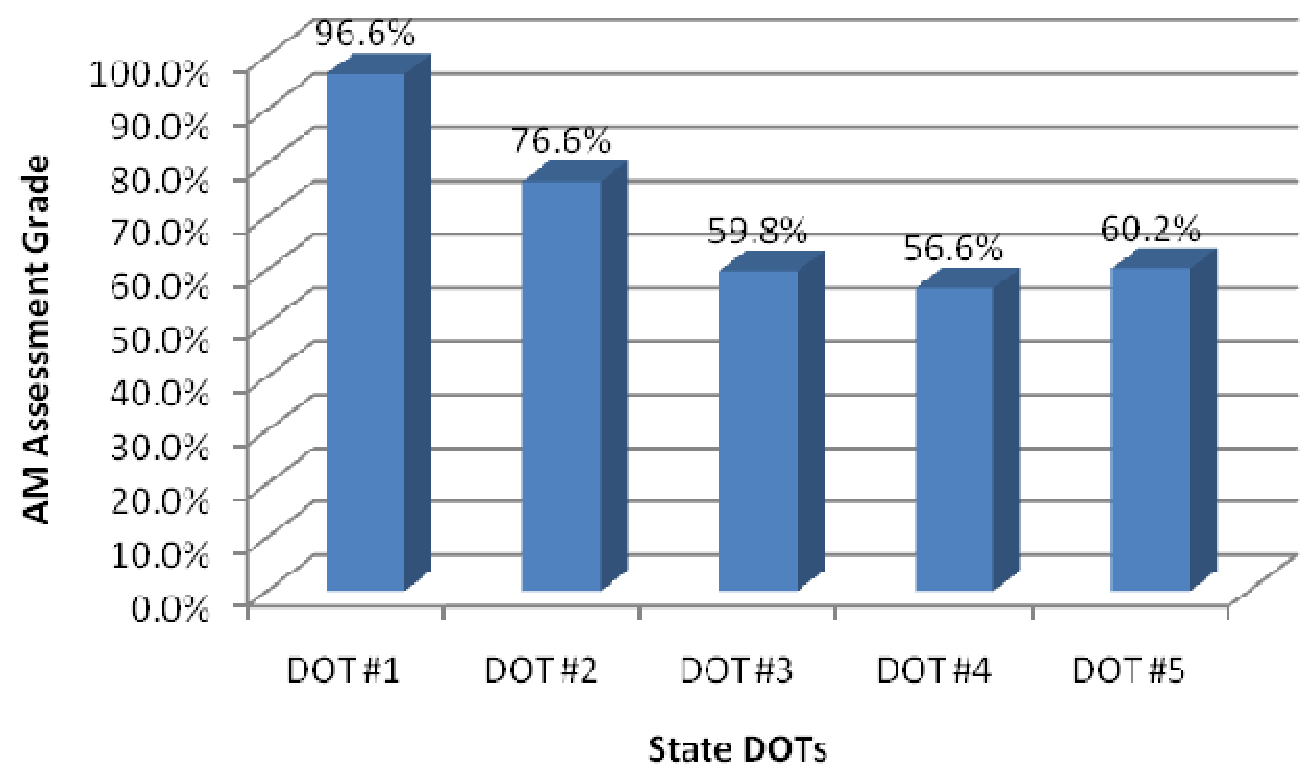

Figure 5.5: Planning \& Programming Grades 


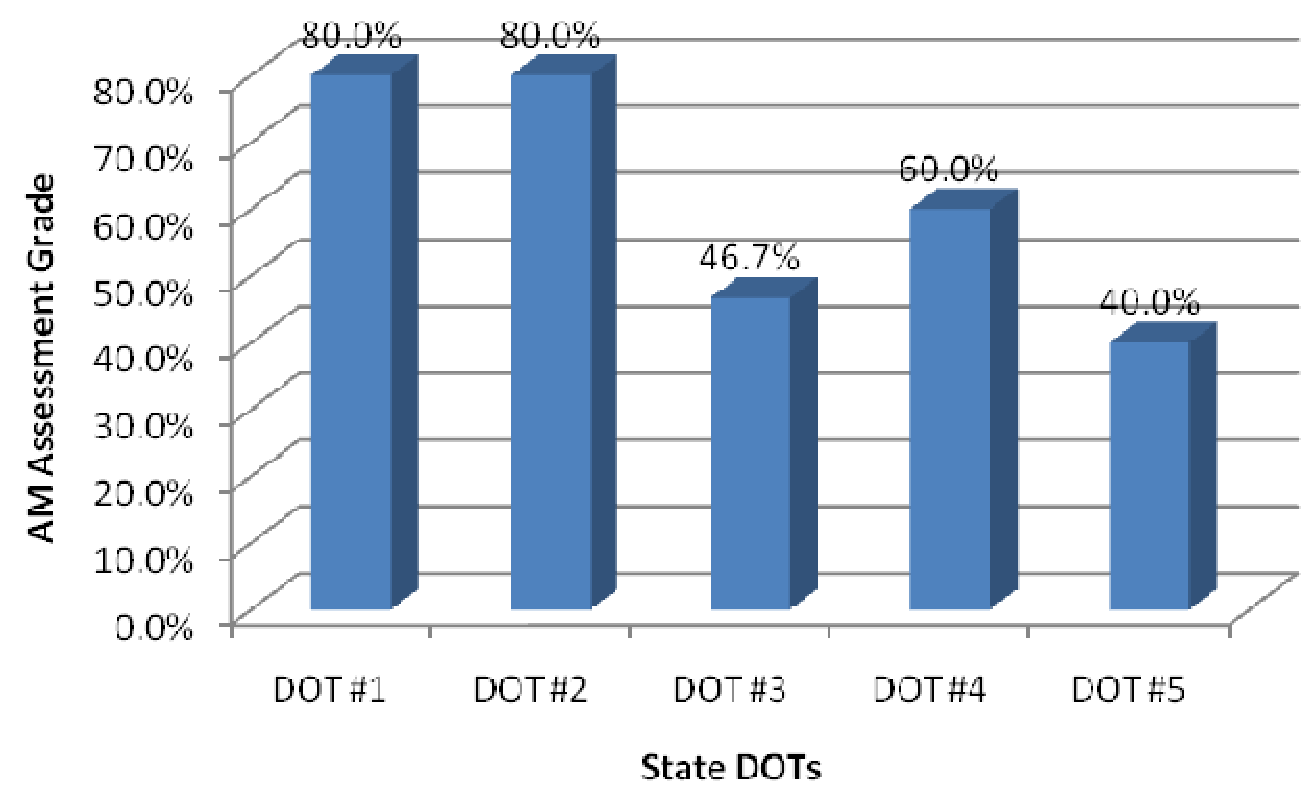

Figure 5.6: Program Delivery Grades

\subsubsection{Sub-Indicator Analysis:}

Analysis of the sub-indicator grades reveals how the top two DOTs separated themselves from the other three in the validation test. The top five most heavily-weighted sub-indicators (1-Policy Goals \& Objectives, 2-Performance Measures \& Targets, 3Program Delivery, 4-Asset Inventory, 5-Decisions Based on Condition Data) demonstrate this separation. The difference between the average grade of the top two DOTs and the average for the remaining three DOTs among these five sub-indicators is significant. Not only are the grades for DOTs \#1 and \#2 much higher in these sub-indicators, but these five sub-indicators represent 58.1 percent of the total weight in the AM Assessment Model. This is a major reason for the separation of DOTs \#1 and \#2 from the others. Table 5.4 shows the grades for all of the sub-indicators for each DOT. Table 5.5 and Figure 5.7 show the gap between the top two DOTs and the remaining three DOTs for the 
top five sub-indicators. Figures 5.8-5.12 graphically represent the grades for each DOT among the top five sub-indicators.

Table 5.4: Sub-Indicator Grades

\begin{tabular}{|c|c|c|c|c|c|}
\hline & DOT \#1 & DOT \#2 & DOT \#3 & DOT \#4 & DOT \#5 \\
\hline Asset Management Champions & $0.0 \%$ & $100.0 \%$ & $70.0 \%$ & $100.0 \%$ & $40.0 \%$ \\
\hline Perception of Asset Management & $80.0 \%$ & $80.0 \%$ & $80.0 \%$ & $60.0 \%$ & $60.0 \%$ \\
\hline Effective Communication & $100.0 \%$ & $80.0 \%$ & $70.0 \%$ & $50.0 \%$ & $80.0 \%$ \\
\hline State Legislative Mandate & $80.0 \%$ & $80.0 \%$ & $40.0 \%$ & $20.0 \%$ & $20.0 \%$ \\
\hline Asset Inventory & $86.7 \%$ & $86.7 \%$ & $53.3 \%$ & $40.0 \%$ & $60.0 \%$ \\
\hline Common Referencing System & $100.0 \%$ & $60.0 \%$ & $80.0 \%$ & $80.0 \%$ & $20.0 \%$ \\
\hline Up-to-Date Condition Data & $100.0 \%$ & $80.0 \%$ & $80.0 \%$ & $80.0 \%$ & $100.0 \%$ \\
\hline Data Management \& Analysis & $86.7 \%$ & $73.3 \%$ & $73.3 \%$ & $46.7 \%$ & $66.7 \%$ \\
\hline Policy Goals \& Objectives & $95.0 \%$ & $80.0 \%$ & $70.0 \%$ & $80.0 \%$ & $45.0 \%$ \\
\hline Performance Measures \& Targets & $84.0 \%$ & $76.0 \%$ & $72.0 \%$ & $48.0 \%$ & $48.0 \%$ \\
\hline Resource Allocation & $90.0 \%$ & $70.0 \%$ & $40.0 \%$ & $50.0 \%$ & $80.0 \%$ \\
\hline Decisions Based on Condition Data & $100.0 \%$ & $80.0 \%$ & $70.0 \%$ & $60.0 \%$ & $50.0 \%$ \\
\hline Program Delivery & $80.0 \%$ & $80.0 \%$ & $46.7 \%$ & $60.0 \%$ & $40.0 \%$ \\
\hline
\end{tabular}


Table 5.5: Separation of the Top Two DOTs

\begin{tabular}{|c||c||c|c|c|c||}
\hline Sub-Indicator & Rank & $\begin{array}{c}\text { Overall } \\
\text { Weight }\end{array}$ & $\begin{array}{c}\text { Avg. Grade of } \\
\text { DOTs \#1 \& } \\
\# 2\end{array}$ & $\begin{array}{c}\text { Avg. Grade of } \\
\text { DOTs \#3, \#4, \& } \\
\# 5\end{array}$ & Difference \\
\hline \hline $\begin{array}{c}\text { Policy Goals \& } \\
\text { Objectives }\end{array}$ & 1 & .171 & $87.5 \%$ & $65 \%$ & 22.5 \\
\hline $\begin{array}{c}\text { Performance } \\
\text { Measures \& Targets }\end{array}$ & 2 & .129 & $80.0 \%$ & $56 \%$ & 24.0 \\
\hline Program Delivery & 3 & .118 & $80.0 \%$ & $48.9 \%$ & 31.1 \\
\hline \hline Asset Inventory & 4 & .082 & $86.7 \%$ & $51.1 \%$ & 35.6 \\
\hline \hline $\begin{array}{c}\text { Decisions Based on } \\
\text { Condition Data }\end{array}$ & 5 & .081 & $90.0 \%$ & $60 \%$ & 30.0 \\
\hline \hline
\end{tabular}

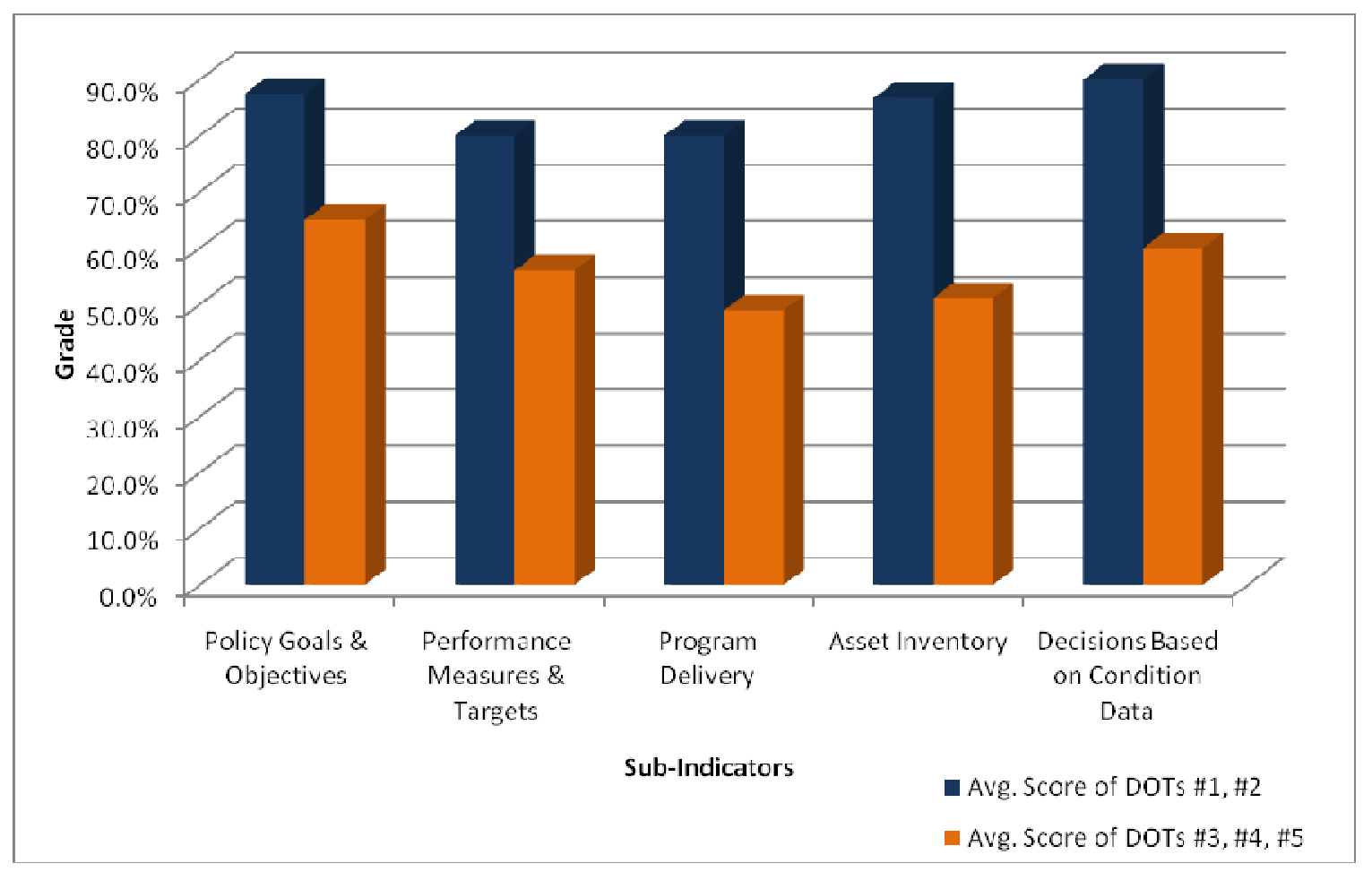

Figure 5.7: Separation of the Top Two DOTs 


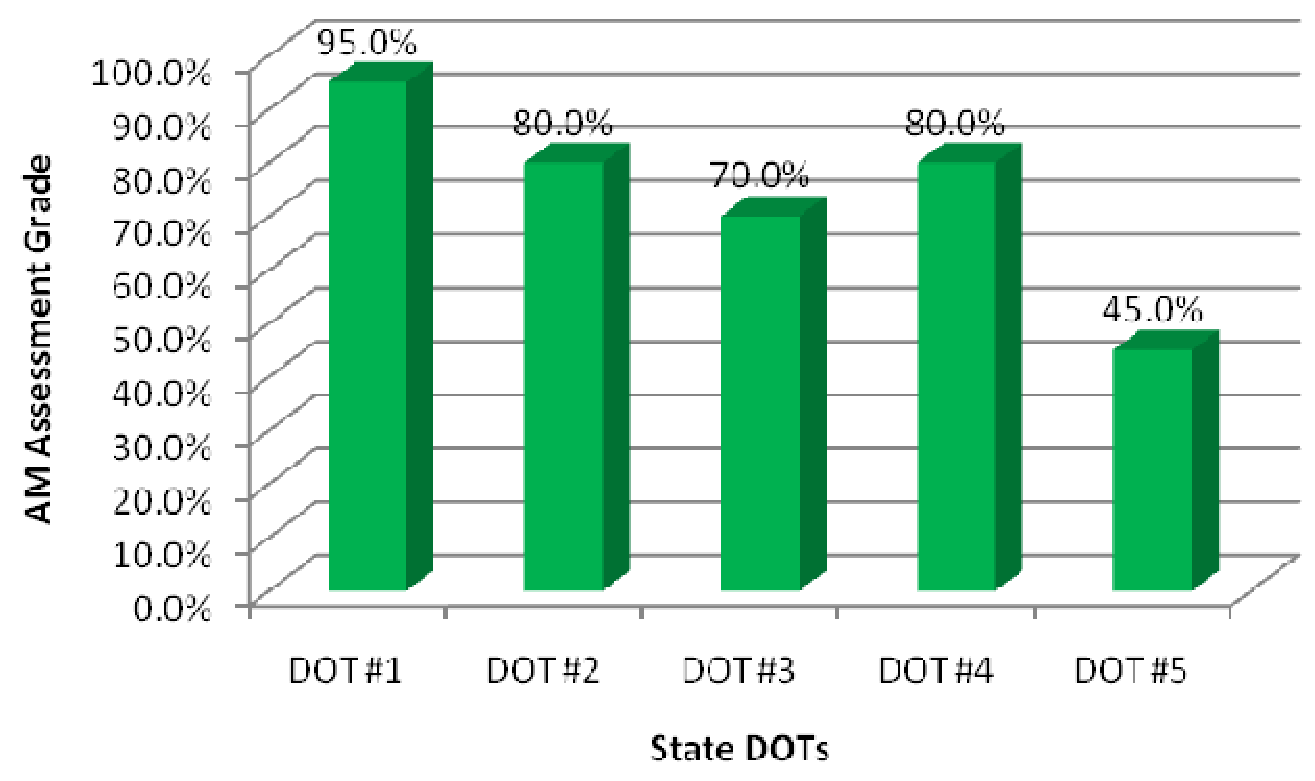

Figure 5.8: Policy Goals \& Objectives Grades (Sub-Indicator)

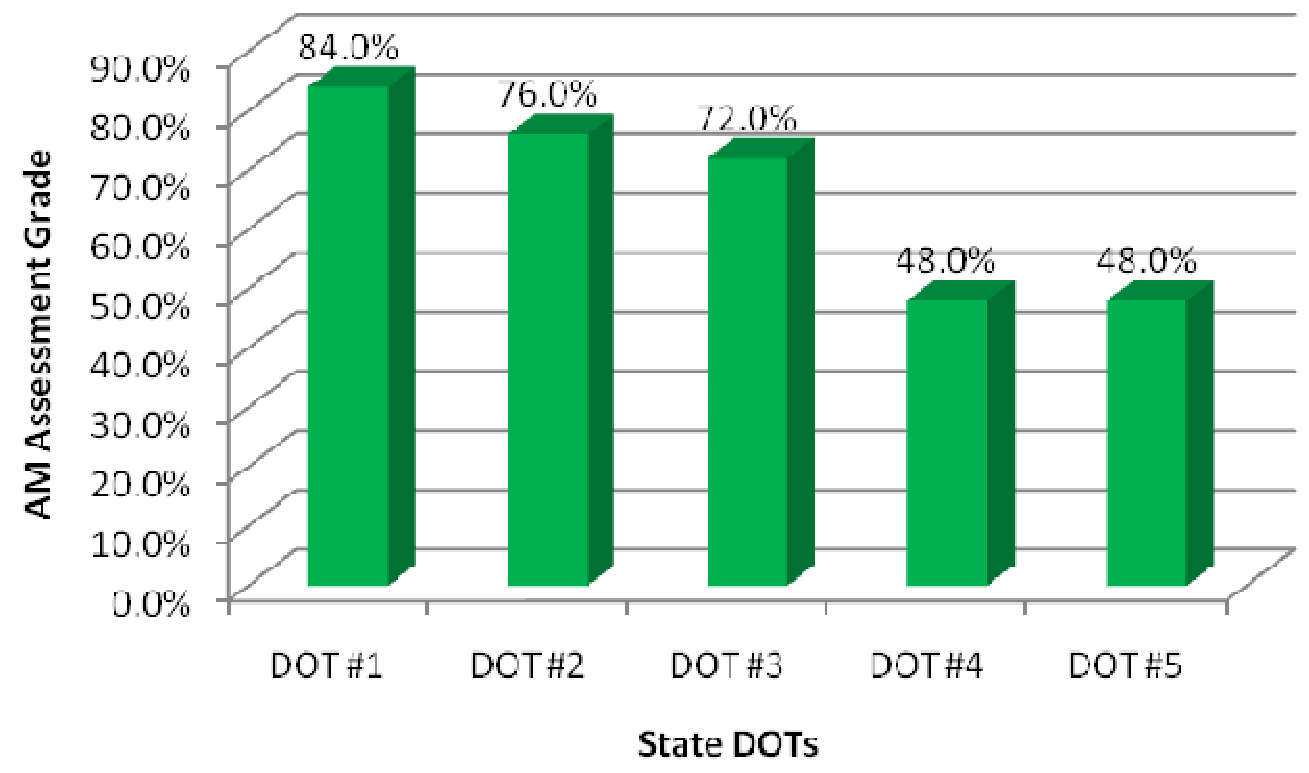

Figure 5.9: Performance Measures \& Targets Grades (Sub-Indicator) 


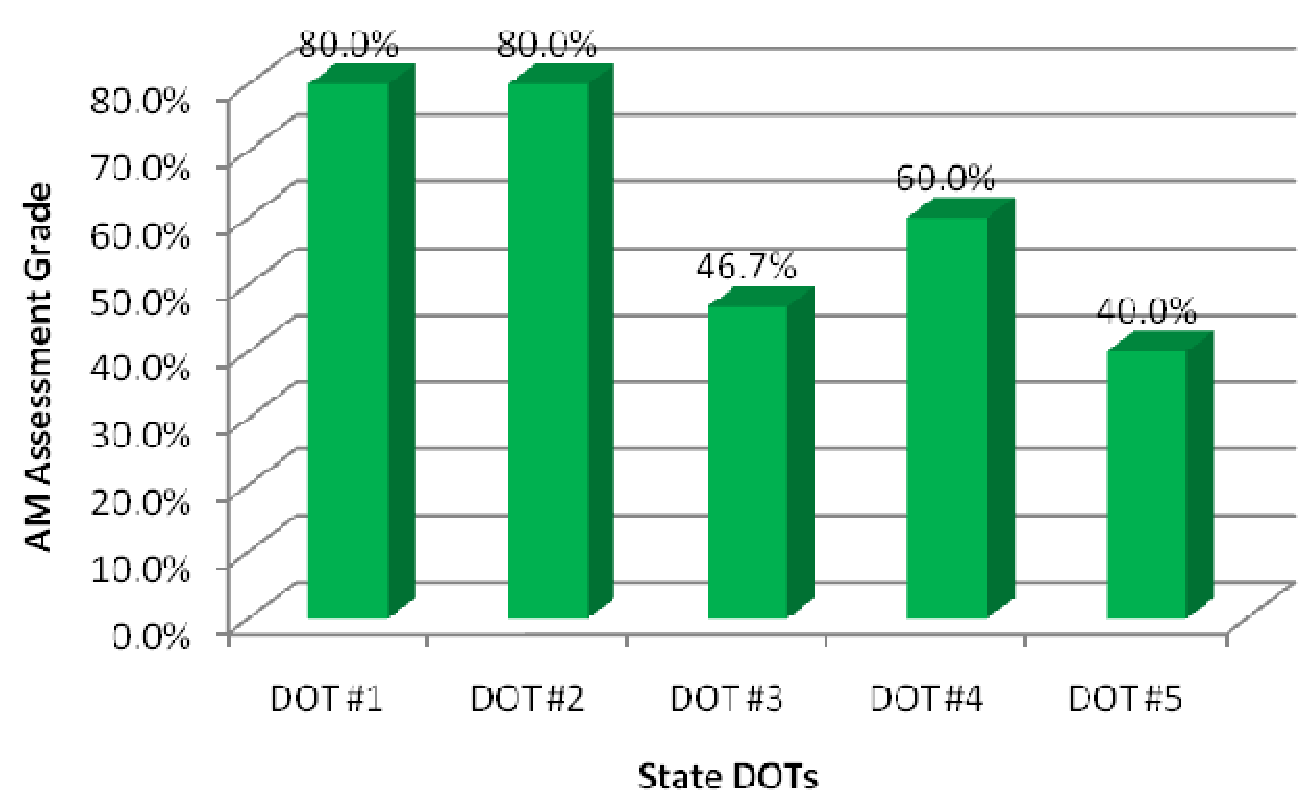

Figure 5.10: Program Delivery Grades (Sub-Indicator)

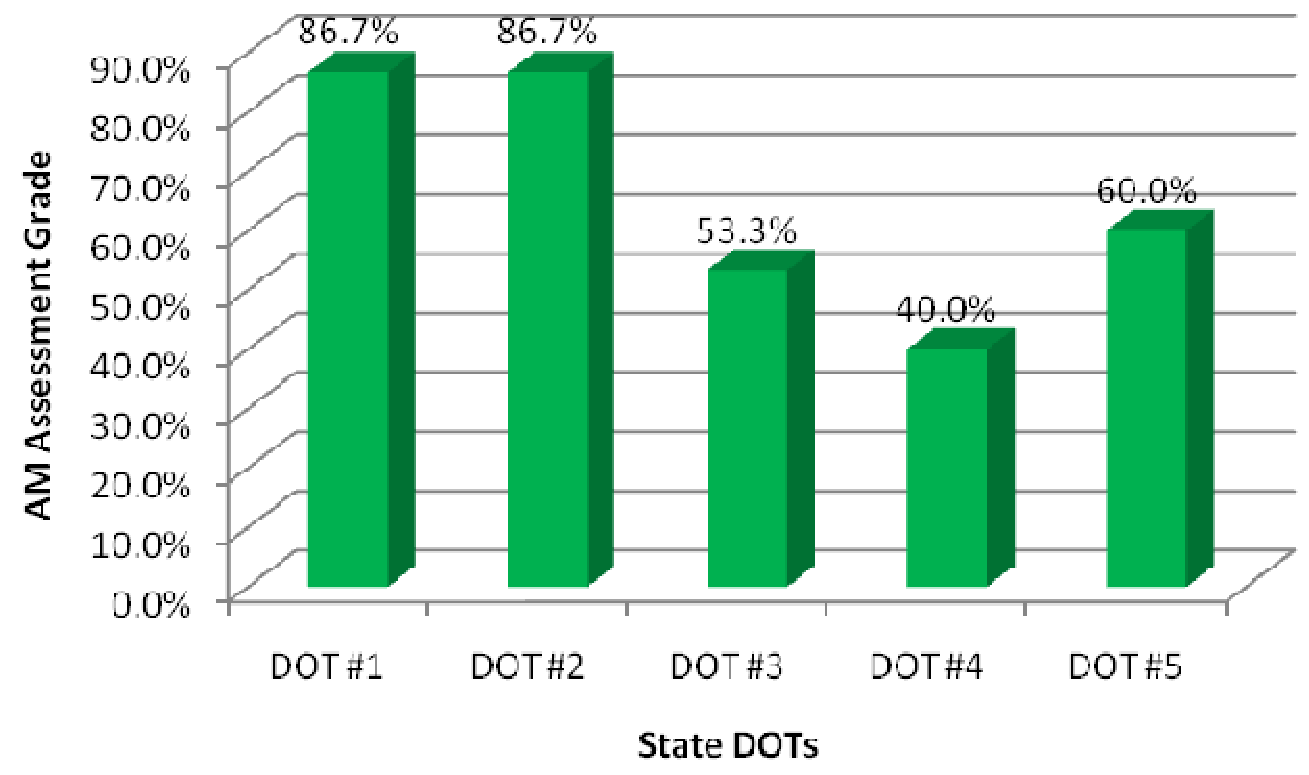

Figure 5.11: Asset Inventory Grades (Sub-Indicator) 


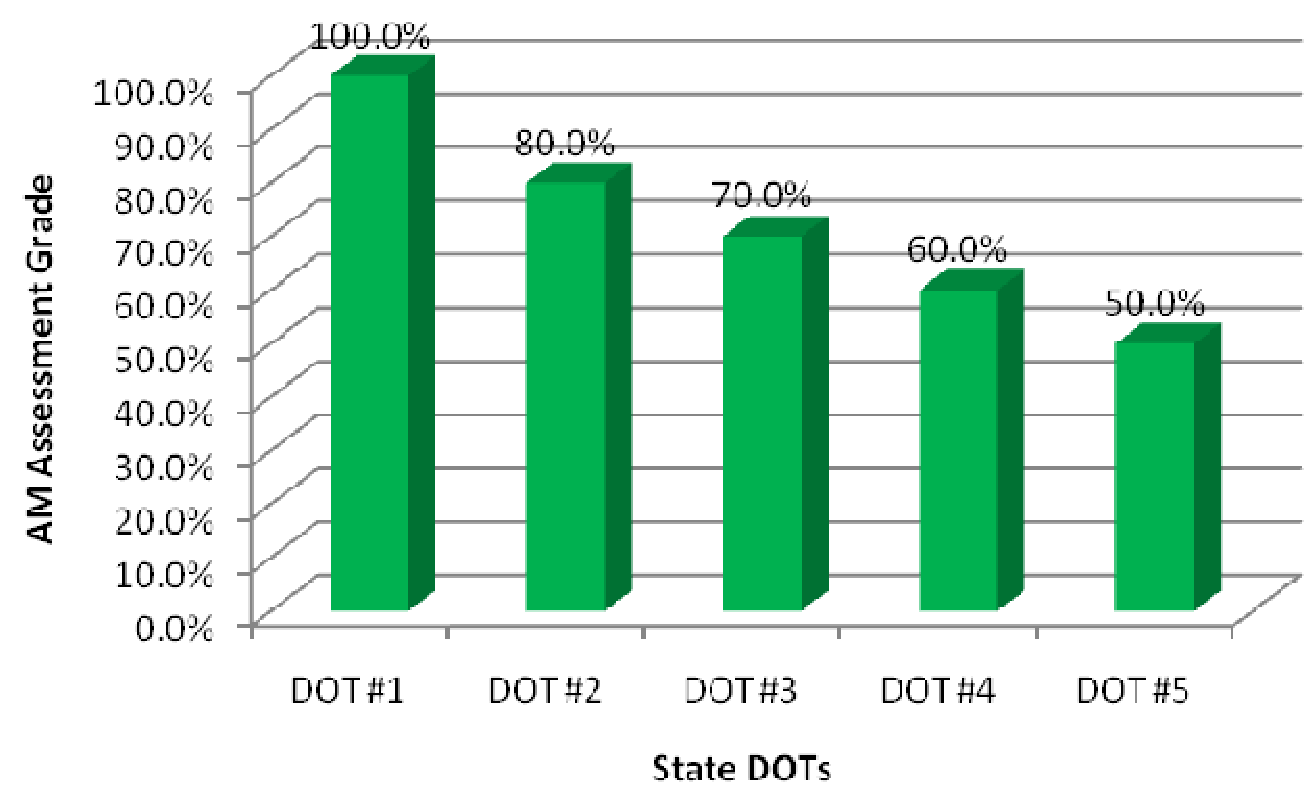

Figure 5.12: Decisions Based on Condition Data (Sub-Indicator)

\subsubsection{Implementation Levels:}

As a result of the validation test, three levels of AM implementation have been identified according to what numerical grade is obtained through the AM Assessment Model. A State DOT will be recognized as having a "High" level of AM implementation if it obtains a grade of 75 percent or better. A grade between 60 and 74 percent will designate a DOT with a "Intermediate" level of AM implementation and a grade below 60 percent will indicate that DOT has a "Low" level of AM implementation. This is shown in Table 5.6. 
Table 5.6: AM Implementation Levels

\begin{tabular}{|c|c|}
\hline \hline Level of AM Implementation & Overall AM Assessment Grade \\
\hline \hline High & $75-100 \%$ \\
\hline \hline Intermediate & $60-75 \%$ \\
\hline Low & $<60 \%$ \\
\hline
\end{tabular}

\subsection{Output of the Asset Management Assessment Model:}

The AM Assessment Model provides an overall grade for a given DOT. This offers a general impression as to what level of AM implementation a DOT is at. For a more detailed breakdown of the assessment grade, the model also supplies scores for each major indicator and sub-indicator. With this information, a DOT can assess their performance and identify their strengths and weakness for each indicator.

In addition to this basic analysis, the model also provides gap analysis that determines which indicators that have the greatest need for improvement. This section of the model allows users to enter scores from a DOT to which they can compare their results. The model then calculates the gaps between the two DOTs for each of the major indicators and sub-indicators. The model also calculates weighted gaps by mulitply the weight for each indicator by is associated gap. This prioritizes the major indicator and sub-indicators according to their weighted gaps. The larger the weighted gap, the greater need for improvement.

For example, consider the DOTs from the validation test. Assume that DOT \#5 wants to improve their AM assessment grade. They know that DOT \#1 is one of the leading agencies in AM and as a result, they want to benchmark DOT \#1. This will allow 
DOT \#5 to determine which of their indicators have the greatest need for improvement when compared to DOT \#1. By taking the scores from DOT \#1 and plugging them into the gap analyis section of the AM Assessment Model, the model will identify the gaps for major indicators and sub-indicators between DOT \#5 and DOT \#1. The output from the model that shows the major indicator gaps is represented in Figure 5.13. According to the weighted gaps, the model also prioritizes which indicators need the most improvement. Figure 5.14 indicates that Policy Goals \& Objectives and Quality Information \& Analysis are the top two major indicators that need improvement if DOT \#5 wants to match the AM assessment grade of DOT \#1. This type of analysis allows DOT \#5 to determine which areas they need to focus their resources in order to improve AM within their agency. Further analysis from the model indicates that while Quality Information \& Analysis needs significant improvement, not all of its sub-indicators require the same magnitude of improvement. Figure 5.15 shows that a Common Referencing System needs significant improvement while Up-to-Date Condition Data needs no improvement when compared to DOT \#1. This information may prove to be valuable to a DOT wishing to know what specific areas they are performing well in and what areas need considerable attention. In summary, the AM Assessment Model provides a DOT with a detailed assessment of which areas they can focus their resources in order to improve AM within their agency. 


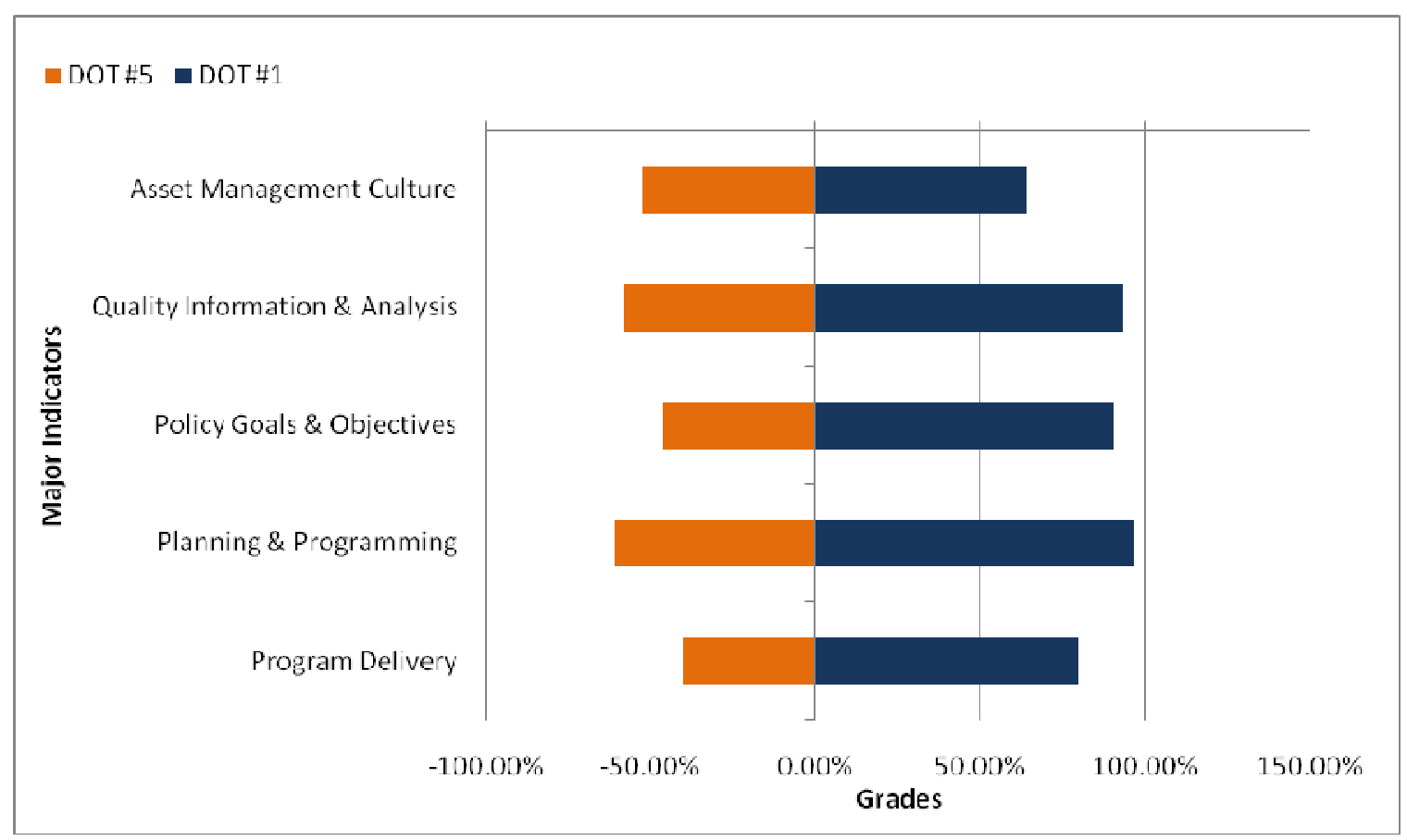

Figure 5.13: Major Indicator Gaps

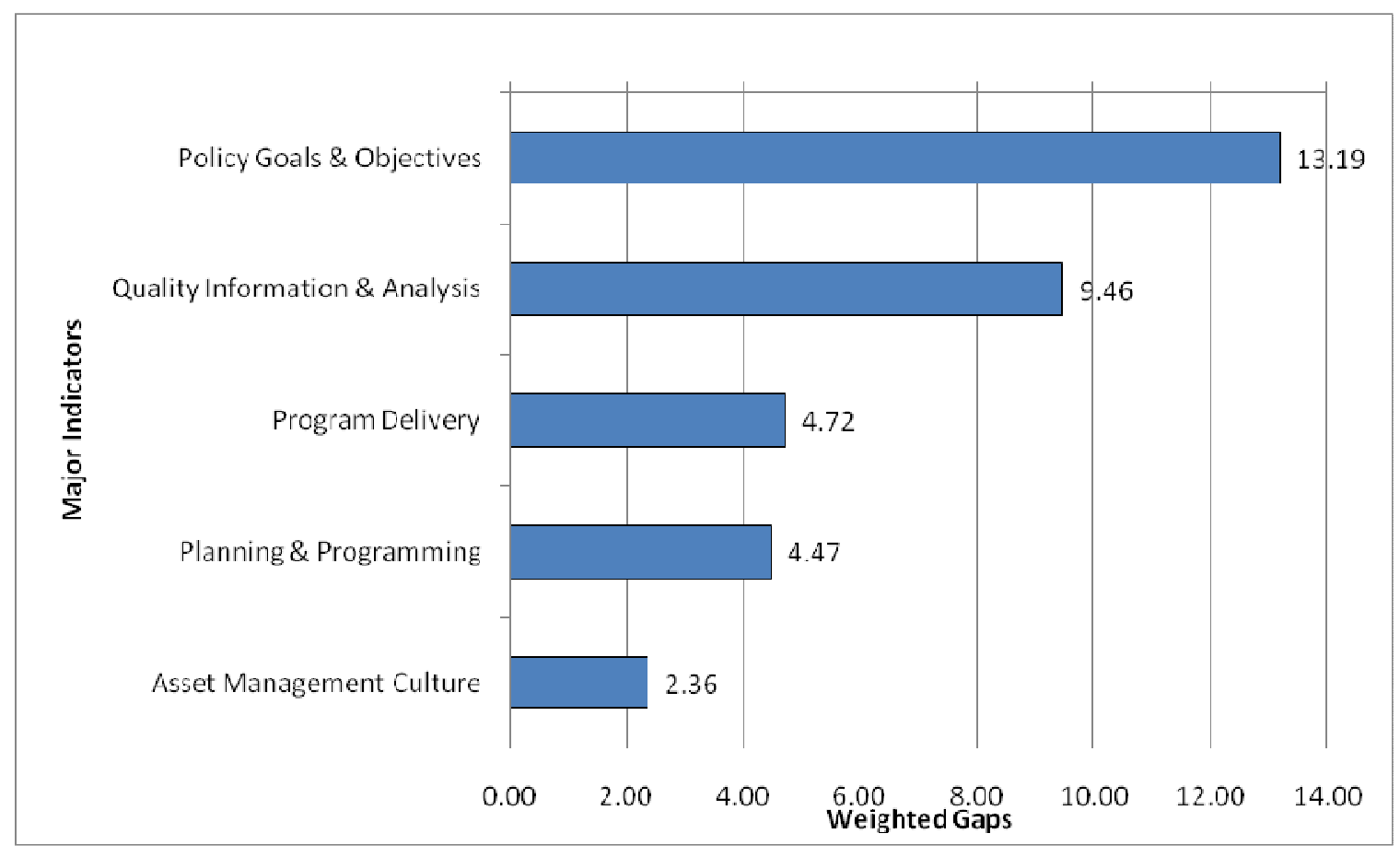

Figure 5.14: Weighted Gaps for the Major Indicators 


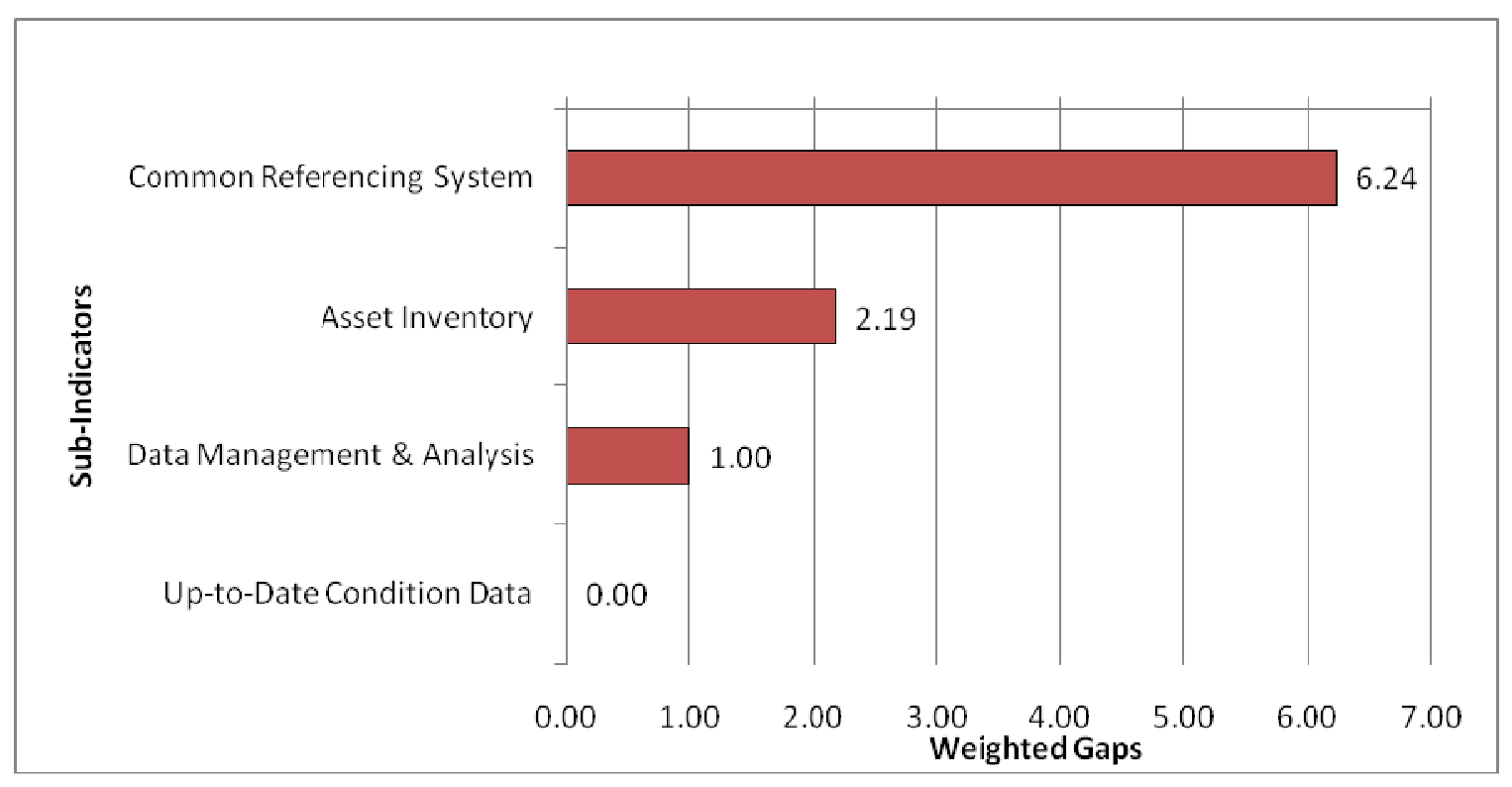

Figure 5.15: Weighted Gaps for Quality Information \& Analysis Sub-Indicators

\subsection{Discussions:}

When the AM Assessment Model was sent to the five DOTs for validation purposes, some unexpected results were received from one of the agencies. The other four agency's results came back within the expected range of values. However, these agencies were known to have either an advanced knowledge of AM or at least very good understanding of AM principles. The agency whose results seemed out of place came from a DOT that was expected to rank near the bottom in AM implementation. This caused the research team to question whether or not all the participants fully understood each of the AM indicators and the statements that accompany them. In a survey as subjective as the AM Assessment Model, it is essential that the participants fully understand the intent of each indicator and each statement. The statements are admittedly broad in nature and the responses subjective themselves. How does one differentiate between 'Strongly Agree' and 'Agree'? The answer depends on the participant's 
understanding of the subject matter and their own honest evaluation of their agency. If the participant does not share the view of AM that the model intends to convey, how can their responses be meaningful?

As a result, the research team decided to perform follow-up phone reviews of each of the five surveys. This allowed the survey participant and the researcher to come to the same understanding for what each statement from the model was intended. After discussing each statement line by line, any necessary changes to the participant's responses were recorded which then reflected changes in that agency's grade. Once this process was completed for each survey, the appropriate changes were made. For DOTs $\# 1-4$, these changes were minor and did not dramatically impact their grade. However, DOT \#5 demonstrated notable changes to its grade. With these changes in place, each of the five DOTs seemed to fit within the expected range of results.

\subsubsection{Improvements:}

In a subjective survey, such as the AM Assessment Model, it is very important to reduce the room for misinterpretation as much as possible. Each AM official, even though they have a good grasp of AM principles, may have a different interpretation or understanding of AM. This was an important lesson learned by the research team and one that was evidenced by having to perform follow-up phone reviews with each of the survey participants. To reduce misinterpretation, it would have been helpful to include a description for each type of response on the AM Assessment Model. The description should be specific to the statement and describe in more detail what each response is 
intended to represent. This would provide better clarity for the participant as to which response is the most appropriate.

While discussing the AM Assessment Model with several AM officials, it was determined that a number of the statements contained language that made it difficult to accurately respond. When addressing major assets, many statements had the word "all" in front of it. The phrase "all major assets" indicates every single major asset. It may be very difficult to answer broad questions about every single major asset. Most AM officials will not have detailed knowledge of all major assets in the strictest definition of the phrase. Several survey participants agreed with this and suggested that the word "all" be removed. By doing this, it enables the participant to respond to statements about the majority of major assets without feeling they are being misleading.

Changes that include adding descriptors to each response type and removing the word "all" from a number of the statements were made to the AM Assessment Model. These changes are a result of the validation procedure and represent the final version of the model. This final version of the AM Assessment Model can be found in Appendix C.

\subsubsection{Ideal Application of the AM Assessment Model:}

The best scenario for implementing the AM Assessment Model is in person. Because the model is subjective, implementing it in person allows for the participant to ask questions that clarify each of the statements. The participant will then acquire the same understanding of AM that is required to accurately address the model. Due to time and budgetary constraints, this study was not able to apply the model to each participant in person. However, an excellent situation to apply this model would be in a group 
setting, such as an AM conference. This would allow for each of the statements from the model to be discussed by AM professionals so that a consensus in the level of understanding could be reached for each one. 


\section{CHAPTER VI}

\section{CONCLUSION}

\subsection{Summary:}

The purpose of this study is to develop a model that measures the level of AM implementation within a State DOT for a benchmarking purpose. In order to satisfy this goal, several research objectives were identified and described in Chapter I. One of the first major objectives was identifying the AM indicators.

$\mathrm{AM}$ indicators are factors that represent the most critical components of an ideal AM system. These indicators were primarily identified through two major types of sources. The first was through an extensive literature review which consisted of examining previous research that had been done in the area of transportation AM. This was composed of research done at the federal level as well as case studies which reported current AM practices at the state level. The second source of identifying AM indicators came from interviewing five leading DOTs in AM. Each of these interviews allowed the DOT officials to disclose their AM practices and discuss what factors they found necessary for successful AM systems.

Weights for the AM indicators were developed based on the assumption that certain indicators are more important than others when implementing an ideal AM system. The methodology chosen to quantify these weights was the Analytic Hierarchy Process (AHP). It was selected because of its widespread and simple approach to 
quantifying subjective items. The nature of this study and the AM indicators themselves are very subjective. As a result, AHP qualified as good methodology to perform this task.

The function of the AM Assessment Model is twofold: 1) to measure the level of AM implementation within a State DOT and thereby serve as an assessment tool and 2) serve as a benchmark so that DOTs may compare their results to other peer agencies. The model was developed in a survey format that allows participants to choose one of six different responses to each one of the statements that characterize the major indicators and sub-indicators. The model was tested on five DOTs of varying AM implementation levels as a validation procedure. If the model is effective, it should return scores that rank each DOT similar to its predetermined level of AM. The results of the validation test showed this to be true. The top two DOTs had scores of 85.6 and 78.1 percent which were higher than the DOTs expected to be in the middle (65.6 and 61.7 percent) which were higher than the DOT expected to finish at the bottom (51.4 percent).

\subsection{Findings and Contributions:}

The first major contribution of this study is the identification of AM Culture as a major indicator. Throughout the literature review and interview process, it became evident that AM was more than just a set of practices and principles. It requires an understanding that AM is the "best way of doing business." Previous studies and AM officials both agree that AM should not be viewed simply as a new program or another competing management system. It should be viewed as a business practice that connects every department and manages the entire system as a whole. This requires buy-in from everyone within the agency as well as a change of culture. This concept is not new in this 
research area; however it has never been formally included as a necessary component of AM. Even though this indicator is subjective, this study identified and included AM Culture as a critical AM indicator. AM Culture was validated as a critical indicator when it received the third highest weight of the five major indicators. This was especially significant considering each of the other four major indicators were already well known to AM officials (they are included in AM framework from the "Transportation Asset Management Guide") whereas AM Culture was not included as a framework component.

The second major contribution is the weighting of the AM indicators based on their level of importance. In previous AM studies, each AM component has been treated with equal weight. The Self-Assessment tool from the "Guide" is the best example of this (Cambridge Systematics 2002). It provides a quick diagnostic tool for agencies to determine where they stand in regards to AM practice. However, each component is given equal weight when calculating one's score. Conversely, this study rests on the assumption that certain $\mathrm{AM}$ indicators are more important than others when implementing an ideal AM system. As a result, AHP was used to quantify the importance and develop weights for the major indicators and sub-indicators.

\subsection{Lessons Learned:}

AHP was used to obtain weights for the AM indicators. This was achieved through the use of a survey which required the respondents to make pairwise comparisons and assign a level of importance of one indicator over another. The use of the survey was employed because of its ease of distribution and cost effectiveness. However, the results of the survey produced large standard deviations for the weights of 
each indicator. This does not necessarily mean that the survey respondents disagree about the importance of each AM indicator due to the fact that there were errors in the way that the survey was administered. It became obvious to the research team that it was very difficult to differentiate between 'Strong Importance' and 'Very Strong Importance,' for example. The definition of each lies in the respondent's interpretation. Therefore, it was concluded that AHP should be administered in person to obtain optimal results. This would allow the participant to gain a much better understanding of each type of response as well as each AM indicator. This could be done either in an individual or a group setting such as an AM conference.

The AM Assessment Model was also administered through the use of a survey. The first time the model was evaluated by each of the five DOTs, there were some errors detected from the responses of one of the DOTs. Based on this, it was determined that some DOTs may not have the same understanding of AM that is required to accurately assess the model. Follow-up phone reviews of each of the participant's responses were conducted so that any discrepancies could be removed and the intended level of understanding of AM could be attained. As a result, it was concluded that the AM Assessment Model would be best administered in person. This could be done in an individual or group setting such as an AM conference.

As a result of the validation procedure, two changes to the AM Assessment Model were deemed necessary. The first had to do with adding descriptions for each type of response. Each participant could easily interpret a response of 'Agree' differently than another, for example. To reduce misinterpretation, a description for each type of response was added. The description is specific to the statement and describes in more detail what 
each response is intended to represent. This provides better clarity for the participant as to which response is the most appropriate.

The second change to the model deals with removing the word "all" from the phrase "all major assets." Most AM officials will not have detailed knowledge of all major assets in the strictest definition of the phrase, and therefore may not be able to accurately assess the statement. By removing the word "all," this allows participants to accurately respond to statements about the majority of major assets without feeling they are being misleading.

\subsection{Future Research:}

Future research that could be done as a follow up to this study would be to improve upon the major indicators and sub-indicators. This may include creating more indicators and providing greater detail to those that already exist. The key in doing this would be to provide major indicators and sub-indicators which illicit responses that separate high, medium, and low DOTs. Creating more detail within the existing indicators may present itself in the form of asking specific questions about different types of assets. This may permit a better and more detailed AM assessment.

Improving the accuracy of the AM indicator weights is another option for future

research. Reaching a more conclusive consensus as to which indicators are the most important for AM implementation would be an excellent contribution. To determine if this is possible, a different assessment methodology may need to be examined as well as how this data is collected. If one or both of these potential research areas are improved upon, the ideal setting for administering an AM Assessment Model would be at an AM 
conference. This would allow a large number of AM experts to participate in the evaluation of the model as well as offer suggestions for improvement. 


\section{REFERENCES}

AECOM Consult, Inc. (2005). Synthesis of Public-Private Partnership Projects for Roads, Bridges, Tunnels From Around the World - 1985-2004, USDOT, FHWA, Washington D.C.

American Association of State Highway and Transportation Officials (AASHTO) and Federal Highway Administration (FHWA) (1997). " $21^{\text {st }}$ Century Asset Management." Proc., Executive Workshop on Asset Management, AASHTO and FHWA, Washington D.C.

Aziz, Ahmed, M. (2007). "Successful Delivery of Public-Private Partnerships for Infrastructure Development." Journal of Construction Engineering and Management, 133(12), 918-931.

Cambridge Systematics, Inc. (2002). "Transportation Asset Management Guide.” Washington D.C.

Cambridge Systematics, Inc. (2005). “Analytical Tools for Asset Management” (NCHRP Report 545). Washington D.C.

Cambridge Systematics, Inc. (2006). "Performance Measures and Targets for Transportation Asset Management." Washington D.C.

Cambridge Systematics, Inc. (2007). "U.S. Domestic Scan Program: Best Practices in Transportation Asset Management." Washington, D.C.

Federal Highway Administration (FHWA). (1999). "Asset Management Primer." Washington, D.C.

Federal Highway Administration (FHWA). (2001). "Data Integration Primer." FHWAIF-01-016, Washington, D.C.

Federal Highway Administration (FHWA). (2002). "Life-Cycle Cost Analysis Primer." FHWA-IF-02-047, Washington, D.C.

Federal Highway Administration (FHWA). (2004). "Report to Congress on PublicPrivate Partnerships." Washington, D.C. 
Federal Highway Administration (FHWA). (2007a). "Asset Management Overview." FHWA-IF-08-008, Washington, D.C.

Federal Highway Administration (FHWA). (2007b). "Comprehensive Asset Management: The North Carolina Experience, Part One.” FHWA-IF-07-010, Washington, D.C.

Federal Highway Administration (FHWA). (2007c). "Comprehensive Asset Management: The Ohio Experience.” FHWA-IF-07-029, Washington, D.C.

Federal Highway Administration (FHWA). (2007d). "Comprehensive Transportation Asset Management: The Washington State Experience." FHWA-IF-07-011, Washington, D.C.

Guerre, Joseph A., Robert, William, E., Bradbury, Alison, and Goodale, Michael (2005). "The Executive Support System of Ontario, Canada: Integrating Pavement and Bridge Management Systems.” Transportation Research Record, 1933, 27-34.

Larson, Charles, D., Skrypczuk, Oleh (2004). "Comprehensive Data Collection to Support Asset Management at Virginia Department of Transportation." Transportation Research Record: Journal of the Transportation Research Board, 1885, 96-103.

Ruikar, K., Anumba, C.J., Carillo, P.M. (2005). "VERDICT - An e-readiness assessment application for construction companies." Automation in Construction, 15 (2006), 98-110.

Saaty, Thomas Lorie. (1980). The Analytic Hierarchy Process. McGraw-Hill, Inc., New York, New York.

Saaty, Thomas L., and Vargas, Luis G. (2001). Models, Methods, Concepts \& Applications of the Analytic Hierarchy Process. Kluwer Academic Publishers, Norwell, Massachusetts.

Zayed, Tarek M, Chang, Luh-Maan. (2002). "Prototype Model for Build-OperateTransfer Risk Assessment." Journal of Management in Engineering, 18(1), 7-16. 
APPENDICES 
APPENDIX A: INDICATOR RANKING SURVEY 


\begin{tabular}{|c|c|}
\hline \multicolumn{2}{|c|}{ Indicators for Successful Asset Management Systems in State DOTs } \\
\hline \multicolumn{2}{|l|}{ Dear transportation agency representative: } \\
\hline \multicolumn{2}{|c|}{$\begin{array}{l}\text { Oklahoma State University is working on a research project entitled "Indicators for Successful Asset } \\
\text { Management Systems in State DOTs." The purpose of this research is to identify indicators that are necessary to } \\
\text { implement a successful Asset Management system for a State DOT. These "indicators" will then help develop } \\
\text { an Asset Management framework that will be used to evaluate State DOTs in terms of their level of Asset } \\
\text { Management implementation. }\end{array}$} \\
\hline \multicolumn{2}{|c|}{$\begin{array}{l}\text { We would like you to participate in this important survey and provide your valuable opinions in transportation } \\
\text { Asset Management for this research. The time to complete this survey is approximately } 25 \text { minutes. We would } \\
\text { like to receive the completed survey form by December } 3,2008 \text {. The completed survey form can be returned via } \\
\text { the following means. }\end{array}$} \\
\hline $\begin{array}{l}\text { Electronic Copy: } \\
\text { Please email to: } \frac{\text { david.jeong@okstate.edu }}{\text { or }} \\
\text { steven.cooksey@okstate.edu } \\
\text { Or, fax to: } 405-744-7554\end{array}$ & $\begin{array}{l}\text { Mail Copy: Dr. David Jeong, Assistant Professor } \\
\text { Oklahoma State University } \\
\text { Civil \& Environmental Engineering Dept. } \\
\text { 207 Engineering South } \\
\text { Stillwater, OK } 74078\end{array}$ \\
\hline \multicolumn{2}{|c|}{$\begin{array}{l}\text { Before you complete this survey, please review Parts I \& II on pages } 2-4 \text {. These sections will enable you to } \\
\text { perform the necessary evaluations and help you accurately make comparisons using the given method. }\end{array}$} \\
\hline \multicolumn{2}{|c|}{$\begin{array}{l}\text { Please feel free to add additional pages if you have suggestions or recommendations related to this survey form. } \\
\text { If you have any questions, please contact me by phone or email. }\end{array}$} \\
\hline \multicolumn{2}{|l|}{ We appreciate your support in advance. } \\
\hline \multicolumn{2}{|l|}{ Sincerely, } \\
\hline $\begin{array}{l}\text { Dr. David Jeong, Ph.D. } \\
\text { Assistant Professor } \\
\text { 207 Engineering South } \\
\text { School of Civil and Environmental Engineering } \\
\text { Oklahoma State University } \\
\text { Stillwater, OK 74078-5033 } \\
\text { Telephone: 405-744-7073 } \\
\text { Fax: 405-744-7554 } \\
\text { Email: david.jeong@okstate.edu }\end{array}$ & $\begin{array}{l}\text { Steven Cooksey } \\
\text { Graduate Assistant } \\
207 \text { Engineering South } \\
\text { School of Civil and Environmental Engineering } \\
\text { Oklahoma State University } \\
\text { Stillwater, OK 74078-5033 } \\
\text { Telephone: 405-744-4698 } \\
\text { Fax: 405-744-7554 } \\
\text { Email: steven.cooksey@okstate.edu }\end{array}$ \\
\hline & \\
\hline
\end{tabular}


Part I: Asset Management Indicators:

These indicators and sub-indicators represent the components necessary to successfully implement an ideal Asset Management system at a State DOT. There are five main Asset Management indicators that each contain a number of sub-indicators. Both indicators and sub-indicators have been identified through a literature review of Asset Management practices as well as interviews with experienced transportation Asset Management practitioners.

\section{A. Asset Management Culture}

Asset Management (AM) is a "way of doing business." It is not merely a new program, but a cultural change that affects every department within the agency. This requires buy-in from everyone involved, especially those at the top of the organization.

1. Asset Management Champions

a. Strong AM leadership is present from top management and decision-makers.

b. The director supports AM as the best "way of doing business."

2. Perception of Asset Management

a. Staff have bought into the fact that AM is the best "way of doing business."

b. Staff understands that AM is not another program, but in fact a change in culture.

3. Effective Communication

a. Strong vertical communication that allows staff to understand the vision of the top managers is present within the agency.

b. Strong horizontal communication between divisions that facilitates tradeoffs between investment areas is present within the agency.

4. State Legislative Mandate

a. There is a legislative mandate that requires some form of AM within the agency.

B. Quality Information and Analysis

In order for an agency to be able to implement Asset Management effectively, it must first know what assets is has, where these assets are located, and what condition these assets are in as well as be able to perform the necessary decisionsupporting analysis.

5. Asset Inventory

a. There is a complete and up-to-date inventory of all major assets.

b. A central relational database is used to store information for all major assets.

c. Staff can easily access all major asset information such as asset type, location, and condition.

6. Common Referencing System

a. Accurate locations for all major assets exist through the use of a common spatial referencing system.

7. Up-to-date Condition Data

a. Consistent and accurate condition data for all major assets is collected on a regular basis.

8. Data Management and Analysis

a. Asset data is managed within a formalized data management framework.

b. There is a person(s) dedicated to managing data analysis software.

c. Past condition data is used to assist performance forecasts of assets through the use of deterioration models.

C. Policy Goals and Objectives

The role of Policy Goals and Objectives is to establish a clear guidance for the remaining steps in the Asset Management framework. This drives the decision-making process of an agency and allows an agency to set priority investment areas.

9. Policy Goals and Objectives (PG\&Os)

a. A priority investment area (system preservation, operations, capacity expansion) exists.

b. PG\&Os guide the agency's overall resource allocation and decision-making process.

c. PG\&Os support a long term life-cycle cost analysis approach rather than a traditional "worst first" approach.

d. PG\&Os are tied directly to performance measures. 
10. Performance Measures and Targets (PM\&Ts)

a. PM\&Ts are present within the agency.

b. PM\&Ts are used to guide project selection.

c. The amount of resources necessary to accomplish specified performance targets is known.

d. PM\&Ts are adjusted according to what resources are available and what is realistically achievable by the agency.

e. PM\&Ts are adjusted to reflect customer perceptions of system performance.

D. Planning and Programming

Once goals and objectives along with performance measures have been established, decision-makers can now perform tradeoff analysis and allocate resources across the network as needed.

11. Resource Allocation

a. Tradeoff analysis across all major assets is performed when making resource allocation decisions.

b. Resources can be moved across districts and assets according to need.

12. Decisions based on Condition Data

a. Resource allocation and programming is based on condition data instead of historical splits.

b. Future projects are programmed on a regular basis according to performance forecasts based on condition data.

\section{E. Program Delivery}

After policies and performance measures have been established, resources have been allocated, and specific projects have been programmed, Program Delivery makes certain that agencies consider the most efficient way to deliver projects.

13. Program Delivery

a. Alternative delivery methods are regularly evaluated in order to select the most efficient and effective method (e.g. design-build, public-private partnerships, etc.).

b. Performance contracts are regularly evaluated (performance specifications, warranties, etc.)

c. Outsourcing options, such as maintenance and operations activities, are regularly evaluated. 
Part II: Comparison Method with Example:

This section explains the method that will be used to compare Asset Management indicators and sub-indicators. This method accomplishes this goal by performing comparisons two at a time. The criteria for making these comparisons are given in Table 1 and illustrated in Figure 1. An example of how to use this comparison system is given in Example 1.

\begin{tabular}{|c|l|l|}
\hline Notation & \multicolumn{1}{|c|}{ Definition } & \multicolumn{1}{c|}{ Explanation } \\
\hline A & Extreme inferiority & $\begin{array}{l}\text { The evidence indicating one activity below another is of the } \\
\text { highest possible order of affirmation }\end{array}$ \\
\hline B & Very strong or demonstrated inferiority & $\begin{array}{l}\text { An activity is very strongly indicated below another; its } \\
\text { inferiority is demonstrated in practice }\end{array}$ \\
\hline C & Strong inferiority & $\begin{array}{l}\text { Experience and judgment strongly indicate one activity below } \\
\text { another }\end{array}$ \\
\hline D & Moderate inferiority & $\begin{array}{l}\text { Experience and judgment slightly indicate one activity below } \\
\text { another }\end{array}$ \\
\hline E & Equal importance & Two activities contribute equally to the objective \\
\hline F & Moderate importance & $\begin{array}{l}\text { Experience and judgment slightly favor one activity over } \\
\text { another }\end{array}$ \\
\hline G & Strong importance & $\begin{array}{l}\text { Experience and judgment strongly favor one activity over the } \\
\text { other }\end{array}$ \\
\hline H & Very strong or demonstrated importance & $\begin{array}{l}\text { An activity is favored very strongly over another; its dominance } \\
\text { is demonstrated in practice }\end{array}$ \\
\hline I & Extreme importance & $\begin{array}{l}\text { The evidence favoring one activity over another is of the highest } \\
\text { possible order of affirmation }\end{array}$ \\
\hline
\end{tabular}

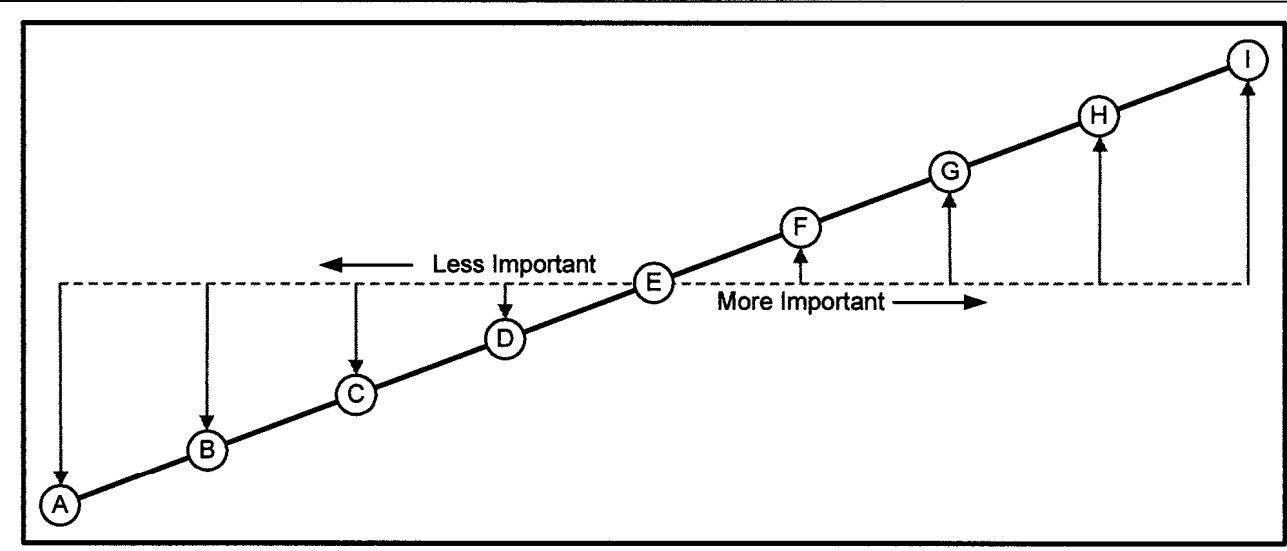

Figure 1. Comparison Scale

Example 1: Factors to consider when buying a new vehicle.

1. What level of importance/inferiority do the following factors exhibit when compared to: Make of the Vehicle?

Let's say that you feel that Gas Mileage is strongly more important than the Make of the Vehicle, but the Color of the Vehicle is very strongly less important than the Make of the Vehicle. Your answer will be as below:

\begin{tabular}{|c|c|c|c|}
\hline & Less important & Equal & More Important \\
\hline Gas Mileage & $\square \mathrm{A} \square \mathrm{B} \square \mathrm{c} \square \mathrm{D}$ & $\square \mathrm{E}$ & $\square \mathrm{F}$ \G $\square \mathrm{H} \square \mathrm{I}$ \\
\hline Color of the Vehicle & $\square \mathrm{A} \triangle \mathrm{B} \square \mathrm{c} \square \mathrm{D}$ & $\square \mathrm{E}$ & $\square \mathrm{F} \square \mathrm{G} \square \mathrm{H} \square \mathrm{I}$ \\
\hline
\end{tabular}


Part III: Compare Asset Management Indicators:

The purpose of this section is to determine which Asset Management (AM) Indicators are more important in successfully implementing an AM system at a State DOT. It is understood that all of the below AM Indicators are very important for successful Asset Management. However, what AM indicators are the most critical for implementing an ideal Asset Management system for a State DOT that is not familiar with Asset Management practices?

This section will compare the five main AM indicators.

Asset Management Indicators:

Please answer the following questions in order to compare the importance of the AM indicators listed below.

- Asset Management Culture

- Quality Information \& Analysis

- Policy Goals \& Objectives

- Planning \& Programming

- Program Delivery

1. What level of importance/inferiority do the following indicators exhibit when compared to: Asset Management Culture?

\begin{tabular}{|l|c|c|c|}
\hline & Less important & Equal & More Important \\
\hline Quality Information \& Analysis & $\square \mathrm{A} \square \mathrm{B} \square \mathrm{c} \square \mathrm{D}$ & $\square \mathrm{E}$ & $\square \mathrm{F} \square \mathrm{G} \square \mathrm{H} \square \mathrm{I}$ \\
\hline Policy Goals \& Objectives & $\square \mathrm{A} \square \mathrm{B} \square \mathrm{c} \square \mathrm{D}$ & $\square \mathrm{E}$ & $\square \mathrm{F} \square \mathrm{G} \square \mathrm{H} \square \mathrm{I}$ \\
\hline Planning \& Programming & $\square \mathrm{A} \square \mathrm{B} \square \mathrm{C} \square \mathrm{D}$ & $\square \mathrm{E}$ & $\square \mathrm{F} \square \mathrm{G} \square \mathrm{H} \square \mathrm{I}$ \\
\hline Program Delivery & $\square \mathrm{A} \square \mathrm{B} \square \mathrm{c} \square \mathrm{D}$ & $\square \mathrm{E}$ & $\square \mathrm{F} \square \mathrm{G} \square \mathrm{H} \square \mathrm{I}$ \\
\hline
\end{tabular}

2. What level of importance/inferiority do the following indicators exhibit when compared to: Quality Information \& Analysis?

\begin{tabular}{|l|c|c|c|}
\hline & Less important & Equal & More Important \\
\hline Policy Goals \& Objectives & $\square \mathrm{A} \square \mathrm{B} \square \mathrm{c} \square \mathrm{D}$ & $\square \mathrm{E}$ & $\square \mathrm{F} \square \mathrm{G} \square \mathrm{H} \square \mathrm{I}$ \\
\hline Planning \& Programming & $\square \mathrm{A} \square \mathrm{B} \square \mathrm{c} \square \mathrm{D}$ & $\square \mathrm{E}$ & $\square \mathrm{F} \square \mathrm{G} \square \mathrm{H} \square \mathrm{I}$ \\
\hline Program Delivery & $\square \mathrm{A} \square \mathrm{B} \square \mathrm{c} \square \mathrm{D}$ & $\square \mathrm{E}$ & $\square \mathrm{F} \square \mathrm{G} \square \mathrm{H} \square \mathrm{I}$ \\
\hline
\end{tabular}

3. What level of importance/inferiority do the following indicators exhibit when compared to: Policy Goals \& Objectives?

\begin{tabular}{|l|c|c|c|}
\hline & Less important & Equal & More Important \\
\hline Planning \& Programming & $\square \mathrm{A} \square \mathrm{B} \square \mathrm{c} \square \mathrm{D}$ & $\square \mathrm{E}$ & $\square \mathrm{F} \square \mathrm{G} \square \mathrm{H} \square \mathrm{I}$ \\
\hline Program Delivery & $\square \mathrm{A} \square \mathrm{B} \square \mathrm{c} \square \mathrm{D}$ & $\square \mathrm{E}$ & $\square \mathrm{F} \square \mathrm{G} \square \mathrm{H} \square \mathrm{I}$ \\
\hline
\end{tabular}

4. What level of importance/inferiority does the following indicator exhibit when compared to: Planning \& Programming?

\begin{tabular}{|l|c|c|c|}
\hline & Less important & Equal & More Important \\
\hline Program Delivery & $\square \mathrm{A} \square \mathrm{B} \square \mathrm{c} \square \mathrm{D}$ & $\square \mathrm{E}$ & $\square \mathrm{F} \square \mathrm{G} \square \mathrm{H} \square \mathrm{I}$ \\
\hline
\end{tabular}


Part IV: Compare Asset Management Sub-Indicators:

The purpose of this section is to determine which AM sub-indicators are more important in successfully implementing an AM system at a State DOT. This section will compare the AM sub-indicators. The sub-indicators are listed below their associated main indicator

A. Asset Management Culture

Please answer the following questions in order to compare the importance of the AM sub-indicators listed below.

- Asset Management Champions

- Perception of Asset Management

- Effective Communication

- State Legislative Mandate

1. What level of importance/inferiority do the following sub-indicators exhibit when compared to: Asset Management Champions?

\begin{tabular}{|l|c|c|c|}
\hline & Less important & Equal & More Important \\
\hline Perception of Asset Management & $\square \mathrm{A} \square \mathrm{B} \square \mathrm{c} \square \mathrm{D}$ & $\square \mathrm{E}$ & $\square \mathrm{F} \square \mathrm{G} \square \mathrm{H} \square \mathrm{I}$ \\
\hline Effective Communication & $\square \mathrm{A} \square \mathrm{B} \square \mathrm{c} \square \mathrm{D}$ & $\square \mathrm{E}$ & $\square \mathrm{F} \square \mathrm{G} \square \mathrm{H} \square \mathrm{I}$ \\
\hline State Legislative Mandate & $\square \mathrm{A} \square \mathrm{B} \square \mathrm{c} \square \mathrm{D}$ & $\square \mathrm{E}$ & $\square \mathrm{F} \square \mathrm{G} \square \mathrm{H} \square \mathrm{I}$ \\
\hline
\end{tabular}

2. What level of importance/inferiority do the following sub-indicators exhibit when compared to: Perception of Asset Management?

\begin{tabular}{|l|c|c|c|}
\hline & Less important & Equal & More Important \\
\hline Effective Communication & $\square \mathrm{A} \square \mathrm{B} \square \mathrm{c} \square \mathrm{D}$ & $\square \mathrm{E}$ & $\square \mathrm{F} \square \mathrm{G} \square \mathrm{H} \square \mathrm{I}$ \\
\hline State Legislative Mandate & $\square \mathrm{A} \square \mathrm{B} \square \mathrm{c} \square \mathrm{D}$ & $\square \mathrm{E}$ & $\square \mathrm{F} \square \mathrm{G} \square \mathrm{H} \square \mathrm{I}$ \\
\hline
\end{tabular}

3. What level of importance/inferiority does the following sub-indicator exhibit when compared to: Effective Communication?

\begin{tabular}{|l|c|c|c|}
\hline & Less important & Equal & More Important \\
\hline State Legislative Mandate & $\square \mathrm{A} \square \mathrm{B} \square \mathrm{c} \square \mathrm{D}$ & $\square \mathrm{E}$ & $\square \mathrm{F} \square \mathrm{G} \square \mathrm{H} \square \mathrm{I}$ \\
\hline
\end{tabular}

B. Quality Information \& Analysis

Please answer the following questions in order to compare the importance of the AM sub-indicators listed below.

- Asset Inventory

- Common Referencing System

- Up-to-Date Condition Data

- Data Management \& Analysis

1. What level of importance/inferiority do the following sub-indicators exhibit when compared to: Asset Inventory?

\begin{tabular}{|l|c|c|c|}
\hline & Less important & Equal & More Important \\
\hline Common Referencing System & $\square \mathrm{A} \square \mathrm{B} \square \mathrm{c} \square \mathrm{D}$ & $\square \mathrm{E}$ & $\square \mathrm{F} \square \mathrm{G} \square \mathrm{H} \square \mathrm{I}$ \\
\hline Up-to-Date Condition Data & $\square \mathrm{A} \square \mathrm{B} \square \mathrm{c} \square \mathrm{D}$ & $\square \mathrm{E}$ & $\square \mathrm{F} \square \mathrm{G} \square \mathrm{H} \square \mathrm{I}$ \\
\hline Data Management \& Analysis & $\square \mathrm{A} \square \mathrm{B} \square \mathrm{c} \square \mathrm{D}$ & $\square \mathrm{E}$ & $\square \mathrm{F} \square \mathrm{G} \square \mathrm{H} \square \mathrm{I}$ \\
\hline
\end{tabular}

2. What level of importance/inferiority do the following sub-indicators exhibit when compared to: Common Referencing System?

\begin{tabular}{|l|c|c|c|}
\hline & Less important & Equal & More Important \\
\hline Up-to-Date Condition Data & $\square \mathrm{A} \square \mathrm{B} \square \mathrm{C} \square \mathrm{D}$ & $\square \mathrm{E}$ & $\square \mathrm{F} \square \mathrm{G} \square \mathrm{H} \square \mathrm{I}$ \\
\hline Data Management \& Analysis & $\square \mathrm{A} \square \mathrm{B} \square \mathrm{C} \square \mathrm{D}$ & $\square \mathrm{E}$ & $\square \mathrm{F} \square \mathrm{G} \square \mathrm{H} \square \mathrm{I}$ \\
\hline
\end{tabular}

3. What level of importance/inferiority does the following sub-indicator exhibit when compared to: Up-to-Date Condition Data?

\begin{tabular}{|l|c|c|c|}
\hline & Less important & Equal & More Important \\
\hline Data Management \& Analysis & $\square \mathrm{A} \square \mathrm{B} \square \mathrm{C} \square \mathrm{D}$ & $\square \mathrm{E}$ & $\square \mathrm{F} \square \mathrm{G} \square \mathrm{H} \square \mathrm{I}$ \\
\hline
\end{tabular}


C. Policy Goals \& Objectives

Please answer the following questions in order to compare the importance of the AM sub-indicators listed below.

- Policy Goals \& Objectives

- Performance Measures \& Targets

1. What level of importance/inferiority does the following sub-indicator exhibit when compared to: Policy Goals \& Objectives?

\begin{tabular}{|l|c|c|c|}
\hline & Less important & Equal & More Important \\
\hline Performance Measures \& Targets & $\square \mathrm{A} \square \mathrm{B} \square \mathrm{C} \square \mathrm{D}$ & $\square \mathrm{E}$ & $\square \mathrm{F} \square \mathrm{G} \square \mathrm{H} \square \mathrm{I}$ \\
\hline
\end{tabular}

D. Planning \& Programming

Please answer the following questions in order to compare the importance of the AM sub-indicators listed below.

- Resource Allocation

- Decisions Based on Condition Data

1. What level of importance/inferiority does the following sub-indicator exhibit when compared to: Resource Allocation?

\begin{tabular}{|l|c|c|c|}
\hline & Less important & Equal & More Important \\
\hline Decisions Based on Condition Data & $\square \mathrm{A} \square \mathrm{B} \square \mathrm{c} \square \mathrm{D}$ & $\square \mathrm{E}$ & $\square \mathrm{F} \square \mathrm{G} \square \mathrm{H} \square \mathrm{I}$ \\
\hline
\end{tabular}

\section{Part V: General Information}

1. Please provide your name

2. Please provide the transportation agency for which you are employed:

3. How many years of experience do you have in the transportation industry?

4. How many years of experience do you have with Asset Management in the transportation industry?

5. What is your position/title within the transportation agency? 
APPENDIX B: ASSET MANAGEMENT ASSESSMENT MODEL, VALIDATION 


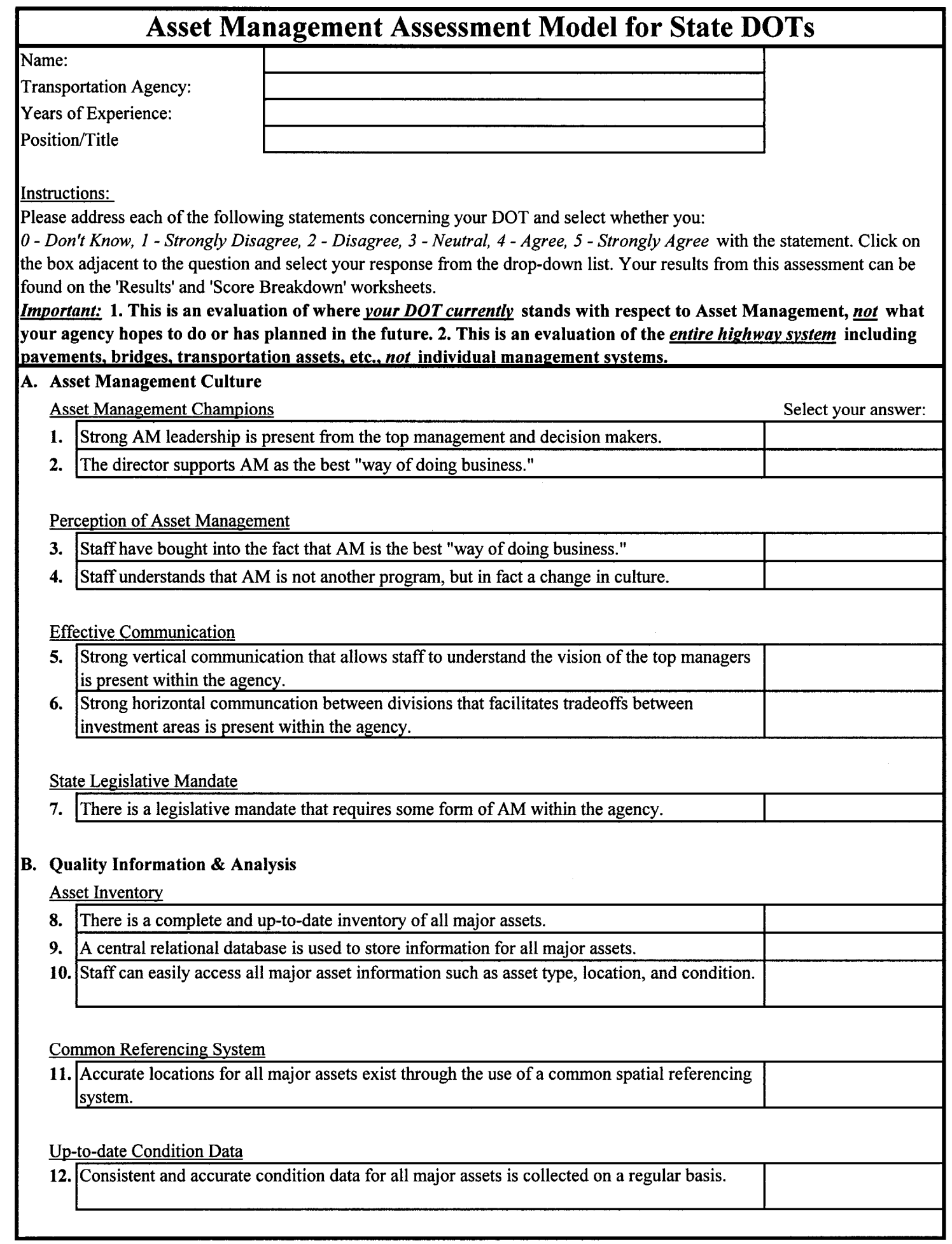


Data Management \& Analysis

13. Asset data is managed within a formalized data management framework.

14. There is a person(s) dedicated to managing data analysis software.

15. Past condition data is used to assist performance forecasts of assets through the use of deterioration models.

C. Policy Goals \& Objectives (PG\&Os)

Policy Goals \& Objectives

16. A priority investment area (system preservation, operations, capacity expansion) exists.

17. PG\&Os guide the agency's overall resource allocation and decision-making process.

18. PG\&Os support a long term life-cycle cost analysis approach rather than a traditional "worst first" approach.

19. PG\&Os are tied directly to performance measures.

Performance Measures \& Targets (PM\&Ts)

20. PM\&Ts are present within the agency.

21. PM\&Ts are used to guide project selection.

22. The amount of resources necessary to accomplish specified performance targets is known.

23. PM\&Ts are adjusted according to what resources are available and what is realistically achievable by the agency.

24. PM\&Ts are adjusted to reflect customer perceptions of system performance.

D. Planning \& Programming

Resource Allocation

25. Tradeoff analysis across all major assets is performed when making resource allocation decisions.

26. Resources can be moved across districts and assets according to need.

Decisions Based on Condition Data

27. Resource allocation and programming is based on condition data instead of historical splits.

28. Future projects are programmed on a regular basis according to performance forecasts based on condition data.

E. Program Delivery

Program Delivery

29. Alternative delivery methods are regularly evaluated in order to select the most efficient and effective method (e.g. design-build, public-private partnerships, etc.).

30. Performance contracts are reguarly evaluated (performance specifications, warranties, etc.)

31. Outsourcing options, such as maintenance and operations activities, are regularly evaluated. 
APPENDIX C: ASSET MANAGEMENT ASSESSMENT MODEL 


\begin{tabular}{l}
\hline \multicolumn{3}{|c|}{ Asset Management Assessment Model for State DOTs } \\
\hline Name: \\
Transportation Agency: \\
Years of Experience: \\
Position/Title
\end{tabular}

Perception of Asset Management

3. Staff have bought into the fact that AM is the best "way of doing business."

4. Staff understands that AM is not another program, but in fact a change in culture.

Effective Communication

5. Strong vertical communication that allows staff to understand the vision of the top managers is present within the agency.

6. Strong horizontal communcation between divisions that facilitates tradeoffs between investment areas is present within the agency.

State Legislative Mandate

7. There is a legislative mandate that requires some form of AM within the agency.

B. Quality Information \& Analysis

Asset Inventory

8. There is a complete and up-to-date inventory of major assets.

9. A central relational database is used to store information for major assets.

10. Staff can easily access major asset information such as asset type, location, and condition.

Common Referencing System

11. Accurate locations for major assets exist through the use of a common spatial referencing system.

Up-to-date Condition Data

12. Consistent and accurate condition data for major assets is collected on a regular basis. 
Data Management \& Analysis

13. Asset data is managed within a formalized data management framework.

14. There is a person(s) dedicated to managing data analysis software.

15. Past condition data is used to assist performance forecasts of assets through the use of deterioration models.

\section{Policy Goals \& Objectives (PG\&Os)}

Policy Goals \& Objectives

16. A priority investment area (system preservation, operations, capacity expansion) exists.

17. PG\&Os guide the agency's overall resource allocation and decision-making process.

18. PG\&Os support a long term life-cycle cost analysis approach rather than a traditional "worst first" approach.

19. PG\&Os are tied directly to performance measures.

Performance Measures \& Targets (PM\&Ts)

20. PM\&Ts are present within the agency.

21. $P M \& T s$ are used to guide project selection.

22. The amount of resources necessary to accomplish specified performance targets is known.

23. PM\&Ts are adjusted according to what resources are available and what is realistically achievable by the agency.

24. PM\&Ts are adjusted to reflect customer perceptions of system performance.

\section{Planning \& Programming}

Resource Allocation

25. Tradeoff analysis across major assets is performed when making resource allocation decisions.

26. Resources can be moved across districts and assets according to need.

Decisions Based on Condition Data

27. Resource allocation and programming is based on condition data instead of historical splits.

28. Future projects are programmed on a regular basis according to performance forecasts based on condition data.

E. Program Delivery

Program Delivery

29. Alternative delivery methods are regularly evaluated in order to select the most efficient and effective method (e.g. design-build, public-private partnerships, etc.)

30. Performance contracts are reguarly evaluated (performance specifications, warranties, etc.)

31. Outsourcing options, such as maintenance and operations activities, are regularly evaluated. 
VITA

\section{Steven Robert Cooksey}

Candidate for the Degree of

Master of Science

Thesis: AN ASSET MANAGEMENT ASSESSMENT MODEL FOR STATE DEPARTMENTS OF TRANSPORTATION

Major Field: Civil Engineering

Biographical:

Personal Data:

Born in Oklahoma City, Oklahoma on April 22, 1985, son of Robert and Peggy

Cooksey.

Education:

Completed the requirements for the Master of Science in Civil Engineering at Oklahoma State University, Stillwater, Oklahoma in May, 2009. Received Bachelor of Science in Civil Engineering at Oklahoma State University, Stillwater, Oklahoma in December, 2007. 
Title of Study: AN ASSET MANAGEMENT ASSESSMENT MODEL FOR STATE DEPARTMENTS OF TRANSPORTATION

Pages in Study: 116

Major Field: Civil Engineering

Scope and Method of Study:

Transportation Asset Management (AM) research has yielded a well-accepted AM framework for State Departments of Transportation (DOTs) to model. However, there have not been any studies that have developed a model that measures the level of AM implementation for a benchmarking purpose. This study accomplishes this by presenting the AM Assessment Model. The model is developed through the identification of the most critical indicators that point to successful practices for an ideal AM system. These indicators are weighed according to their level importance by using the Analytic Hierarchy Process (AHP).

Findings and Conclusions:

While identifying the indicators, a new indicator, AM Culture was identified and recognized as significant by some of the leading AM experts in the United States.

By developing a model that incorporates weights for each of the indicators, a more precise tool to assess the level of AM implementation within a State DOT has been presented. 